\title{
RADIOACTIVITY ANALYSIS \\ IN \\ NIOBIUM ACTIVATION FOILS
}

$$
\angle A-S U B-96-71
$$

June 1995

\author{
Submitted by: \\ Dr. Gary E. Mueller \\ Nuclear Engineering \\ University of Missouri \\ Rolla, MO 65401
}

Submitted to:

Dr. Walter Sommer

Los Alamos National Laboratory

Los Alamos, NM 87545

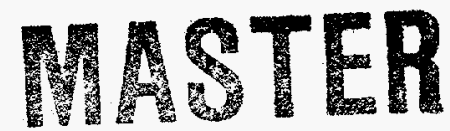

DISTRIBUTION OF THIS DOCUMENT IS UNLMMITED 


\section{DISCLAMMER}

Portions of this document may be illegible in electronic image products. Images are produced from the best available original document. 


\section{DISCLAIMER}

This report was prepared as an account of work sponsored by an agency of the United States Government. Neither the United States Government nor any agency thereof, nor any of their employees, make any warranty, express or implied, or assumes any legal liability or responsibility for the accuracy, completeness, or usefulness of any information, apparatus, product, or process disclosed, or represents that its use would not infringe privately owned rights. Reference herein to any specific commercial product, process, or service by trade name, trademark, manufacturer, or otherwise does not necessarily constitute or imply its endorsement, recommendation, or favoring by the United States Government or any agency thereof. The views and opinions of authors expressed herein do not necessarily state or reflect those of the United States Government or any agency thereof. 


\section{TABLE OF CONTENTS}

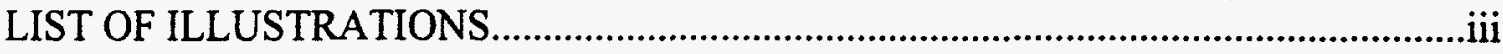

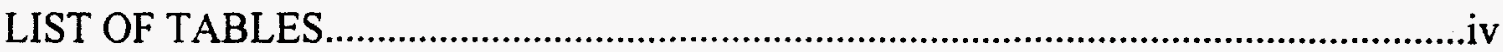

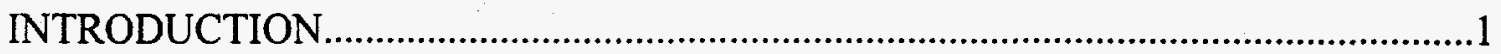

NIOBIUM ACTIVITY MEASUREMENTS ........................................................

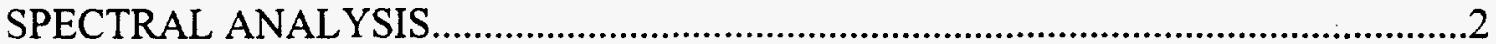

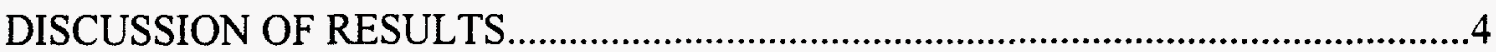

REFERENCES

APPENDICES

I. UMC Nb-93m Activity Determination...........................................................

II. Figures for Beam History, Differential Neutron Flux,

and Neutron Energy Flux.

III. Reactions, Corrected Saturated Activities, and Integrated

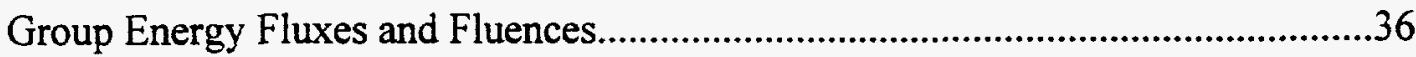

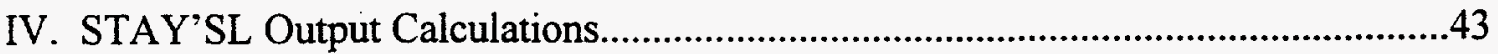




\section{LIST OF ILLUSTRATIONS}

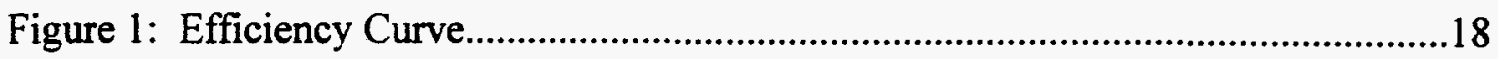

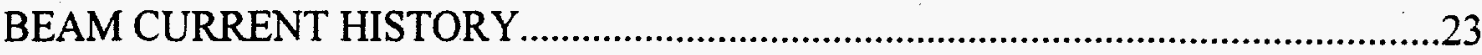

1-1 TO 1-5 DIFFERENTIAL NEUTRON FLUX ................................................24

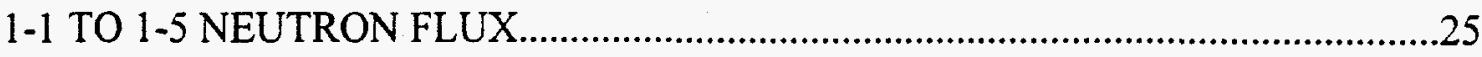

2-1 TO 2-5 DIFFERENTIAL NEUTRON FLUX.................................................26

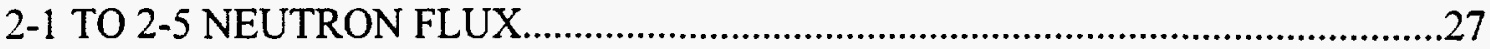

3-1 TO 3-5 DIFFERENTIAL NEUTRON FLUX...............................................28

3-1 TO 3-5 NEUTRON FLUX.....................................................................29

4-1 TO 4-5 DIFFERENTIAL NEUTRON FLUX.....................................................30

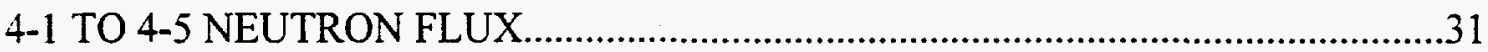

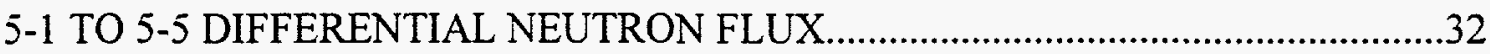

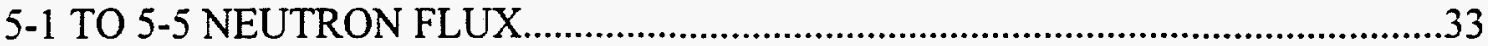

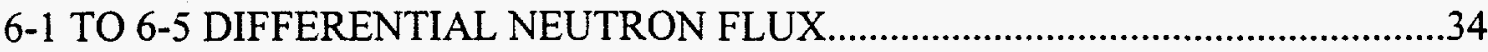

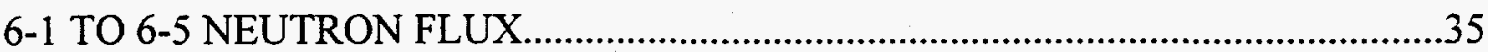




\section{LIST OF TABLES}

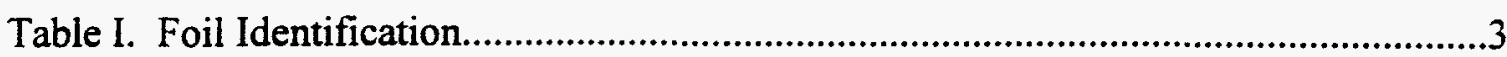

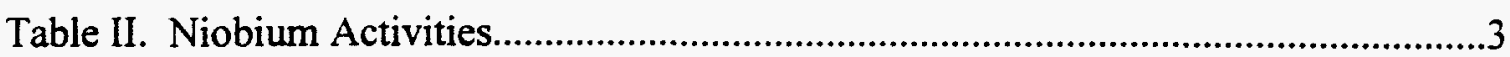

Table III. Summary of Total Neutrons....................................................................4

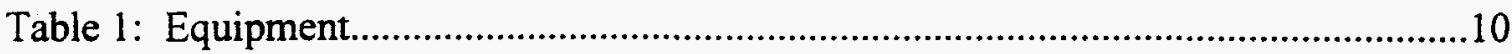

Table 2: Detector Efficiency in POS 1 Geometry for Gamma-X Detector ....................11

Table 3: Attenuation Coefficient Calculation using Foil 2A .......................................12

Table 4: Miscellaneous Information Used in this Project...........................................13

Table 5: Standard Deviation and Percent Error of counts per second

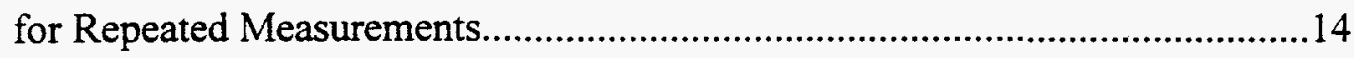

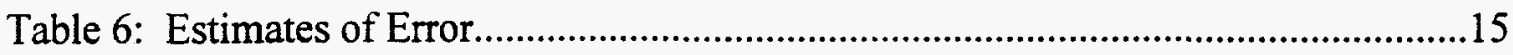




\section{INTRODUCTION}

The motivation for this study was to measure and analyze the activity of six (6) niobium ( $\mathrm{Nb}$ ) foils (the $\mathrm{X}$-rays from an internal transition in $\mathrm{Nb}-93 \mathrm{~m}$ ) and apply this information with previously obtained activation foil data. The niobium data was used to determine the epi-thermal to $\mathrm{MeV}$ range for the neutron spectrum and fluence. The foil activation data was re-evaluated in a spectrum analysis code (STAY'SL) to provide new estimates of the exposure at the Los Alamos Spallation Radiation Effect Facility (LASREF).

The activity of the niobium foils was measured and analyzed at the University of Missouri-Columbia (UMC) under the direction of Professor William Miller. The spectrum analysis was performed at the University of Missouri-Rolla (UMR) by Professor Gary Mueller.

This study was supported by the Los Alamos National Laboratory (LANL) and the University of California through subcontract number $4425 \mathrm{M} 0014-3 \mathrm{G}$ for $\$ 25,448$ under the supervision of Dr. Walter Sommer.

\section{NIOBIUM ACTIVITY MEASUREMENTS}

Six niobium foils were irradiated in a neutron flux during the period, August 1 to October 13, 1992 at LASREF at the Los Alamos Meson Physics Facility (LAMPF). Gamma-ray spectrum measurements were determined in July, 1993 at LAMPF. However, fast neutron activation of niobium produces radioactive ${ }^{93 \mathrm{~m}} \mathrm{Nb}$ from the activation reaction ${ }^{93} \mathrm{Nb}\left(\mathrm{n}, \mathrm{n}^{\prime}\right){ }^{93 \mathrm{~m}} \mathrm{Nb}$. The half-life of ${ }^{93 \mathrm{~m}} \mathrm{Nb}$ is 16.13 years and has $\mathrm{K} \mathrm{x}-$ ray emission energies at 16.6 and $18.6 \mathrm{keV}$. As a result, the gamma-ray measurements at LAMPF did not provide any data about the niobium activation reaction. 
In the fall of 1994, the six niobium foils were shipped from LANL to UMC to be measured and analyzed using the niobium activation reaction ${ }^{93} \mathrm{Nb}\left(\mathrm{n}, \mathrm{n}^{\prime}\right){ }^{93 \mathrm{~m}} \mathrm{Nb}$. The complete results from the UMC measurements and analysis are given in Appendix I.

Table I and II summarize the UMC results that are used in the spectral analysis. Table II shows that there are three foils for each sample except for foil 2A. Based on the weights of the foils and their activities the following six foils are used in the spectral analysis: $1 \mathrm{~A}, 2 \mathrm{~A}, 3 \mathrm{C}, 4 \mathrm{C}, 5 \mathrm{~A}$, and $6 \mathrm{~A}$.

\section{SPECTRAL ANALYSIS}

Five foils $(\mathrm{Ni}, \mathrm{Co}, \mathrm{Fe}, \mathrm{Cu}, \mathrm{Nb})$ were irradiated in a neutron flux during the period, August 1 to October 13,1992 at LAMPF. The neutron irradiation was done at the six positions of $1-1$ to $1-5,2-1$ to $2-5,3-1$ to $3-5,4-1$ to $4-5,5-1$ to $5-5$, and $6-1$ to $6-5$. The recorded beam current history is shown in Appendix II. The average integrated beam current for this irradiation is 307.17 microAmps. The beam current history is used to correct the activity for decay during irradiation using the $\mathrm{BCF}$ [1] computer code. The corrected activities along with an initial spectrum, activation cross sections, and variance covariance information are used as input to the STAY'SL [2] computer program. The STAY'SL code calculates the most probable neutron energy spectrum using a leastsquare technique. Figures for the differential neutron flux and the neutron energy flux obtained from the STAY'SL calculations for the six positions are given in Appendix II. The reactions analyzed and their corrected saturated activities for the six positions are listed in Appendix III. Also shown is the difference between the measured activity, the adjusted activity calculated by STAY'SL, and the $90 \%$ energy limits. In addition, Appendix III lists the integrated group energy fluxes, fluences for the different neutron energy groups and percentage of neutrons above $20 \mathrm{MeV}$. The complete results of STAY'SL calculations for each of the six positions of 1-1 to 1-5 through 6-1 to 6-5 are given in Appendix IV. 
Table I. Foil Identification

\begin{tabular}{|c|c|}
\hline MURR ID & LANL ID \\
\hline IA & shot 1 \\
\hline $2 \mathrm{~A}$ & shot 2 \\
\hline $3 \mathrm{~A}$ & shot 4 \\
\hline $4 \mathrm{~A}$ & shot 3 \\
\hline $5 \mathrm{~A}$ & shot 5 \\
\hline $6 \mathrm{~A}$ & shot 6 \\
\hline
\end{tabular}

Table II. Niobium Activities

\begin{tabular}{|c|c|c|c|}
\hline Sample & $\begin{array}{c}\text { Mass } \\
(\mathrm{mg})\end{array}$ & $\begin{array}{c}\text { E.O.I. Activity } \\
(\mu \mathrm{Ci} / \mathrm{mg})\end{array}$ & $\begin{array}{c}\text { Estimated } \\
\% \text { Error }\end{array}$ \\
\hline IA & 16.80 & 0.252 & $20 \%$ \\
\hline 1B & 17.04 & 0.246 & $20 \%$ \\
\hline IC & 17.08 & 0.236 & $20 \%$ \\
\hline 2A & 16.88 & 0.734 & $20 \%$ \\
\hline 3A & 17.42 & 0.376 & $20 \%$ \\
\hline 3B & 14.28 & 0.460 & $20 \%$ \\
\hline 3C & 16.85 & 0.398 & $20 \%$ \\
\hline 4A & 17.30 & 0.187 & $20 \%$ \\
\hline 4B & 17.33 & 0.199 & $20 \%$ \\
\hline 4C & 16.73 & 0.199 & $20 \%$ \\
\hline 5A & 16.97 & 0.491 & $20 \%$ \\
\hline 5B & 16.79 & 0.492 & $20 \%$ \\
\hline 5C & 16.94 & 0.488 & $20 \%$ \\
\hline $6 \mathrm{~A}$ & 16.93 & 0.342 & $20 \%$ \\
\hline 6B & 16.91 & 0.343 & $20 \%$ \\
\hline $6 \mathrm{C}$ & 16.75 & 0.335 & $20 \%$ \\
\hline
\end{tabular}




\section{DISCUSSION OF RESULTS}

The niobium activation reaction ${ }^{93} \mathrm{Nb}\left(\mathrm{n}, \mathrm{n}^{\prime}\right){ }^{93 \mathrm{~m}} \mathrm{Nb}$ provides useful neutron measurements in an energy range that has not been previously presented. As shown in the $90 \%$ energy limits in Appendix III, the activation reaction for the niobium provides values from $0.5 \mathrm{MeV}$ to approximately $6 \mathrm{MeV}$. This new measurement is significant in that both the number of neutrons and their energy are substantial in this region. As a result, these neutrons will have a significant effect on the total number of neutrons and the number neutrons above $20 \mathrm{MeV}$. The measured niobium activation data provide the needed resolution in this energy region to more accurately describe the overall neutron spectra.

Establishing the neutron activity in this new energy region provides new estimates of the number of neutrons above $20 \mathrm{MeV}$. As shown in Appendix III these values vary from 3.89 to 1.72 percent of the total. There seems to be a correlation between the total number of neutrons and the number of neutrons above $20 \mathrm{MeV}$. That is, as the total number of neutrons decreases the percentage of neutrons above $20 \mathrm{MeV}$ also decreases. This trend is shown below in Table III.

Table III. Summary of Total Neutrons

\begin{tabular}{|c|c|}
\hline $\begin{array}{c}\text { TOTAL NUMBER OF NEUTRONS } \\
\mathrm{n} / \mathrm{cm}^{2} \text {-s-mA }\end{array}$ & $\begin{array}{c}\text { PERCENT NEUTRON ABOVE 20 MeV } \\
\text { percent }\end{array}$ \\
\hline $2.884 \mathrm{E}+13$ & 3.89 \\
\hline $1.508 \mathrm{E}+13$ & 2.48 \\
\hline $1.312 \mathrm{E}+13$ & 2.33 \\
\hline $1.230 \mathrm{E}+13$ & $\mathbf{2 . 2 4}$ \\
\hline $8.598 \mathrm{E}+12$ & 1.82 \\
\hline $7.730 \mathrm{E}+12$ & 1.72 \\
\hline
\end{tabular}

The bold data indicates foil package $5-1$ to $5-5$ which should be used with caution since the peak identification is ambiguous.

\section{REFERENCES}

[1] Davidson, D.R. Neutron and proton dosimetry at the LAMPF 800-MeV proton accelerator, Ph.D. Dissertation, Iowa State University, Ames, IA (1990).

[2] Perey, F.G., Least-squares dosimetry unfolding: The program STAY'SL, ORNL/TM-6062. ORNL (1977). 


\section{APPENDIX I}

UMC Nb-93m Activity Determination 
TO:

Dr. William H. Miller

March 8, 1995

FROM: $\quad$ Norman A. Kahler

SUBJECT: Niobium foil Sample identification

Attached are three tables that show various information from the LANL data and the MURR data for the Y-88 peak. As shown at the bottom of the MURR data table, approximately 510 days have passed since the data from LANL was taken. Because only an approximate decay correction is going to be applied; the shot 1 EOI time of 262 days was added to the 510 days to find the EOI time for the MURR readings. The activity of the MURR data is simply

$$
\begin{aligned}
& \text { activity }=\frac{C P S \cdot \mathrm{e}^{\lambda \cdot \mathrm{t}}}{\varepsilon \cdot \text { yield }} \\
& \text { where: } \\
& \varepsilon: \quad \text { The efficiency at } 0.898 \mathrm{MeV} \\
& \mathrm{t}: \quad \text { The EOI time }(772 \text { days }) \\
& \text { CPS: The counts per second } \\
& \text { yield: The yield fraction. }(0.93)
\end{aligned}
$$

Sample $2 \mathrm{~A}$ or shot 2 are known to be the same because only one foil was contained in this particular vial. It is also obvious from the data because this particular sample is the most radioactive of all the samples. The comparison that was performed basically compares the magnitude of the activity in Becquerel $(\mathrm{Bq})$ to sample 2 or shot 2 for the respective data. It is concluded from the attached tables that the following MURR ID samples have the following LANL ID.

\begin{tabular}{|c|c|c|c|}
\hline MURR ID & $\begin{array}{c}\text { Percent difference } \\
\text { from Sample 2A }\end{array}$ & LANL ID & $\begin{array}{c}\text { Percent difference } \\
\text { from Shot 2 }\end{array}$ \\
\hline IA & $85.2 \%$ & shot 1 & $84.8 \%$ \\
\hline $2 \mathrm{~A}$ & $0.0 \%$ & shot 2 & $0.0 \%$ \\
\hline $3 \mathrm{~A}$ & $63.4 \%$ & shot 4 & $62.7 \%$ \\
\hline $4 \mathrm{~A}$ & $87.2 \%$ & shot 3 & $86.8 \%$ \\
\hline $5 \mathrm{~A}$ & $55.9 \%$ & shot 5 & $56.4 \%$ \\
\hline $6 \mathrm{~A}$ & $71.3 \%$ & shot 6 & $70.8 \%$ \\
\hline
\end{tabular}

To further justify the results, the activity of the MURR data was ratio to the LANL data for the above determined identifications. As the attached ratio table indicates, the ratio of the MURR ID samples to the appropriate LANL ID samples were all approximately 1.5. This justifies the selection of the matching MURR ID to the LANL ID because the value of there ratios were all about the same. Although why the activities were different by 1.5 has not been determined. The purpose of this analysis was to determine which MURR ID went with the appropriate LANL ID. Therefore, no further analysis was done to determine why this difference of 1.5 exists. 
LANL DATA

\begin{tabular}{|c|c|c|c|} 
Name-1D & $D P M$ & Bq & Percent difference from shot 2| \\
\hline shot 1 & $1.559 E+07$ & $2.598 E+05$ & 0.848050682 \\
shot 2 & $1.026 E+08$ & $1.710 E+06$ & 0 \\
shot 3 & $1.351 E+07$ & $2.252 E+05$ & 0.868323587 \\
shot 4 & $3.824 E+07$ & $6.373 E+05$ & 0.627290448 \\
shot 5 & $4.470 E+07$ & $7.450 E+05$ & 0.564327485 \\
shot 6 & $2.994 E+07$ & $4.990 E+05$ & 0.708187135 \\
\hline
\end{tabular}

\begin{tabular}{|c|c|c|c|}
\multicolumn{3}{|c|}{ MURR DATA } \\
Name-ID & CPS & Bq & Percent difference from 2A \\
\hline $1 A$ & $3.160 E+01$ & $3.833 E+05$ & 0.852336449 \\
$2 A$ & $2.140 E+02$ & $2.596 E+06$ & 0 \\
$3 A$ & $7.840 E+01$ & $9.509 E+05$ & 0.63364486 \\
$4 A$ & $2.740 E+01$ & $3.323 E+05$ & 0.871962617 \\
$5 A$ & $9.430 E+01$ & $1.144 E+06$ & 0.559345794 \\
$6 A$ & $6.140 E+01$ & $7.447 E+05$ & 0.713084112 \\
\hline
\end{tabular}

22-Nov-94 30-Jun-93 510 days

EOI of the LANL data 262 days

$Y-88$ half life $\quad 106.61$ days

yield fraction of $Y-88 \quad 0.93$

efficiency @ $0.898 \mathrm{MeV} \quad 0.134$

\begin{tabular}{|l|r|}
\hline \multicolumn{2}{|c|}{ RATIOS } \\
\hline $1 A /$ shot 1 & $1.475 E+00$ \\
$2 A /$ shot 2 & $1.518 E+00$ \\
$3 A /$ shot 4 & $1.492 E+00$ \\
$4 A /$ shot 3 & $1.476 E+00$ \\
$5 A /$ shot 5 & $1.535 E+00$ \\
$6 A /$ shot 6 & $1.492 E+00$ \\
\hline
\end{tabular}




\title{
Nb-93m ACTIVITY \\ DETERMINATION \\ IN \\ NIOBIUM FOILS
}

\author{
by \\ Mr. NORMAN KAHLER \\ Dr. WILLIAM H. MILLER \\ Dr. MICHAEL D. GLASCOCK
}

\section{DEPARTMENT OF NUCLEAR ENGINEERING \\ AND \\ RESEARCH REACTOR CENTER}

UNIVERSITY OF MISSOURI - COLUMBIA

Columbia, MO 65211

JAN 30, 1994 


\section{TABLE OF CONTENTS}

INTRODUCTION

EQUIPMENT

PROCEDURE

Calibration of Detector:

Counting the Foils: 2

Attenuation Calculation: 2

Data Handling:

Repeatability of Ifeasurements:

Error Analvisis:

SUMMARY AND CONCLUSION

APPENDLX A: Activities measured in Niobium Foils $\quad 10$

REFERENCES 12

FIGURES

Figure 1: Efficiency Curc 9

\section{TABLES}

Table 1: Equipment 1

Table 2: Detector Efficiency in POS 1 Gcometn for Gamma-X Detector 2

Table 3: Attenuation Coefficient Calculation using Foil 2A 3

Table 4: Miscellancous Information Used in this Project 4

Table 5: Standard Deviation and Percent Error of counts per second for Repeated Measurements 5

Table 6: Estimates of Error $\quad 6$ 


\section{INTRODUCTION}

The purpose of this experiment is to measure the activity of $\mathrm{Nb}-93 \mathrm{~m}$ in the niobium foils.

To accomplish this task, the University of Missouri Research Reactor was enlisted to do this experiment because of the availability of high resolution low energy germanium detectors. The following is a description of how the results contained in Appendix A were attained.

\section{EQUIPMENT}

The basic equipment used in this experiment is contained in the following table.

\begin{tabular}{|c|c|c|}
\hline Name of Equipment & Model Number & Serial Number \\
\hline $\begin{array}{c}\text { Digital VAX station 3100 M38 } \\
\text { (w/ Digital Storage Expansion) }\end{array}$ & WS42A-DA* & AB023065II* \\
\hline ORTEC Bias Supply & ORTEC 459 & - \\
\hline ORTEC Amplifier & ORTEC 572 & - \\
\hline Nuclear Data ADC & ND 570 & - \\
\hline Nuclear Data AIM & ND 556 & - \\
\hline ORTEC Nim Bin & ORTEC 4001A BIN & - \\
\hline $\begin{array}{c}\text { EG\&G ORTEC Gamma-X HPGe } \\
\text { coaxial detector }\end{array}$ & GMX-30200-P & 31 -TN10688A \\
\hline $\begin{array}{c}\text { Analytics, calibrated Am-241 } \\
\text { Point Source }\end{array}$ & - & $49076-156$ \\
(HP-199)
\end{tabular}

Note: A blank was left if the model or serial number was unavailable or unknown.

\section{Table 1: Equipment}

\section{PROCEDURE}

\section{Calibration of the Detector:}

To begin the experiment certain materials were needed to calibrate the Gamma-X HPGe coaxial detector. The source purchased for low energy calibration was an Am-24l point source. This source was sealed in mylar tape and centered in the window of an aluminum plate. The MURR designation for this source is HP-199. Using this source and a mixed gamma source (HP-5) an efficiency curve from $10 \mathrm{keV}$ to $4000 \mathrm{keV}$ was derived in the POS 1 geometry (one inch from the face of the detector) as seen in Figure 1. Table 2 lists values used to define the efficiency curve. 


\begin{tabular}{|c|c|}
\hline $\begin{array}{c}\text { Gamma Energy } \\
\text { (keV) }\end{array}$ & Efficiency \\
\hline 10.00 & 0.0100 \\
13.90 & 0.0199 \\
26.35 & 0.0779 \\
33.21 & 0.0922 \\
59.54 & 0.0883 \\
59.90 & 0.0865 \\
88.00 & 0.0835 \\
122.00 & 0.0718 \\
$300.00^{\circ}$ & 0.0340 \\
662.00 & 0.0168 \\
1173.00 & 0.0095 \\
1332.00 & 0.0085 \\
$4000.00^{\circ}$ & 0.0030 \\
\hline Note: By interpolation the efficiency at $_{16.6 \mathrm{keV}}=$ & 0.02288 \\
$18.6 \mathrm{keV}=$ & 0.02574 \\
\hline
\end{tabular}

*These efficiencies were interpolated or extrapolated

Table 2: Detector Efficiency in POS 1 Geometry for Gamma-X Detector

Counting the Foils:

Before the foil samples were counted. each sample was weighed on a digital scale (Mettler AT 261 Delta Range, calibrated 5-4-94). Extreme caution was taken not to touch the foils and to keep the handling time to a minimum. The foils were mounted at the center of sample cards, similar to the HP-199 source, with tape used to fix the sample in position. To verify that the tape did not attenuate the foil, measurements were made with tape on one side of the foil and then with tape on both sides of the foil. The resuits showed no statistical difference. Thus, all the foils were taped to the sample cards with tape on both sides. The foils were identified by a number and a letter. The number designated the vial they arrived in and the letter designated the individual foil from that vial. Each sample was then counted in the same position that the calibration source had been counted in (i.e. the POS 1 geometry). The foils were each counted for 15 minutes at this geometry. Attenuation Calculation:

Attenuation factors were determined by counting sample $2 \mathrm{~A}$ with and without a $0.03 \mathrm{~cm}$ attenuation foil. The amount of radiation attenuated through the $0.03 \mathrm{~cm}$ foil is correlated to a coefficient called an attenuation coefficient. This coefficient can be used to determine how much radiation is attenuated at any thickness through the source foil. The measurements with and without 
the attenuation foil were taken for 15 minutes. The following table shows the results from these measurements and the corresponding results from the attenuation coefficient calculations.

Data

\begin{tabular}{|c|c|c|c|}
\hline & $\begin{array}{c}\text { Energy } \\
\text { (keV) }\end{array}$ & Peak Area & $\begin{array}{c}\text { Correeted Live } \\
\text { Time (sec) }\end{array}$ \\
\hline Unattenuated & 16.6 & 187638 & \\
( Io ) & 18.6 & 45336 & 750 \\
\hline Attenuated & 16.6 & 5503 & \\
(I) & 18.6 & 2616 & 763 \\
\hline
\end{tabular}

Numerical

\begin{tabular}{|c|c|c|}
\hline $\begin{array}{c}\text { Energy } \\
(\mathbf{k e V})\end{array}$ & $\mathbf{I / I}$ & $\begin{array}{c}\mu(\mathbf{1} / \mathbf{c m}) \\
\ln (\mathbf{I} / \mathbf{l o}) /(-\mathbf{x})\end{array}$ \\
\hline 16.6 & 0.0288 & 118.21 \\
18.6 & 0.0567 & 95.65 \\
\hline
\end{tabular}

Table 3: Attenuation Coefficient Calculation using Foil $2 A$

The numerical calculation used the following formula to calculate $\mu$, the attenuation coefficient.

$$
\frac{I}{I_{0}}=\mathrm{e}^{-\mu x}
$$

$I_{o}=$ unattenuated counts per second (peak area / corrected live time)

$I$ = attenuated counts per second (peak area / corrected live time)

$\mu=$ attenuation coefficient

$\mathrm{x}=$ attenuation foil thickness $(0.03 \mathrm{~cm})$

See reference 1 .

Data Handling:

There was a possibility of Ta-182 induced fluorescence $\mathrm{x}$-rays causing interference and an error in the results. This possible interference needed to be considered. If the Ta-182 activity is less than $2 \%$ of the $\mathrm{Nb}-93 \mathrm{~m}$ activity in a $0.1 \mathrm{~mm}$ thick niobium foil, the correction of Ta- 182 induced fluorescence will be less than about $5 \%$. The correction is less for lighter foils and greater for heavier foils. ${ }^{2}$ Through measurement, it was determined the amount of Ta-182 activity in the samples, at the time of measurement, was less than $2 \%$ of the Nb-93m activity in the $\sim 0.3 \mathrm{~mm}$ thick foils. Thus, no correction was required for Ta-182 activity. A problem could also occur if Niobium metal has a Tantalum content greater than $5 \mathrm{ppm}$ before irradiation. ${ }^{2}$ From the chemical analysis information. of the Niobium foil. that was received with the attenuation foil, the Tantalum content was less than $5 \mathrm{ppm}$. Note, the attenuation foil was assumed to be from the same batch of Niobium foils that were irradiated. 
Another problem that had to be addressed was with how the computer software performed

its automatic peak search on low-energy peaks. The computer program did not always automatically find the correct peaks. The use of a manual interactive peak search was required to correct the problem.

Table 4 lists some of the information needed to calculate the $\mathrm{Nb}-93 \mathrm{~m}$ activity. One piece of information of interest from Table 4 is the assumed end of irradiation (E.O.I.). None of the information received to do this experiment explicitly gave an E.O.I. time. However, the time listed was assumed by using the delta time listed on the MicroVAX-RAYGUN output we received.

\begin{tabular}{|c|c|}
\hline $\begin{array}{l}\text { Assumed End of Irradiation } \\
\text { Date of Measurement at MURR } \\
\text { Decay Time (days) }\end{array}$ & $\begin{array}{c}11-\text { Oct-92 } \\
28-\text { Nov-94 } \\
778\end{array}$ \\
\hline $\begin{array}{l}\text { Half-life of Nb-93m } \\
\text { Decay Correction }\end{array}$ & $\begin{array}{c}16.13 \text { years } \\
1.096\end{array}$ \\
\hline $\begin{array}{l}\mathrm{K} \text { X-ray emission probability }{ }^{2} \\
\text { K-alpha energy of } \mathrm{Nb}-93 \mathrm{~m}^{2} \\
\text { K-beta energy of } \mathrm{Nb}-93 \mathrm{~m}^{2}\end{array}$ & $\begin{array}{c}0.115 \text { per decay } \\
16.6 \mathrm{keV} \\
18.6 \mathrm{keV}\end{array}$ \\
\hline Thickness of attenuation foil & $0.3 \mathrm{~mm}$ \\
\hline
\end{tabular}

\section{Table 4: Miscellaneous Information Used in this Project}

Assuming the $\mathrm{Nb}-93 \mathrm{~m}$ activity was homogeneously distributed throughout the foil. The overall attenuation was determined by integrating $\exp (-\mu \mathrm{x})$ across the thickness of the foil as the following derivation illustrates.

$$
\begin{aligned}
& \frac{I(x)}{I_{0}}=e^{-\mu x} \\
& I(x)=I_{0} \cdot e^{-\mu x} \\
& I(x) \cdot \int_{0}^{x} d x=I_{0} \cdot \int_{0}^{x} e^{-\mu x} d x \\
& I(x) \cdot x=I_{0} \cdot \frac{1}{\mu} \cdot\left(1-e^{-\mu x}\right. \\
& I_{0}=\frac{I(x) \cdot x \cdot \mu}{\left(1-e^{-\mu x}\right)}
\end{aligned}
$$

$\mathrm{I}_{\mathrm{o}}=$ unattenuated counts per second (peak area / corrected live time)

$I$ = attenuated counts per second (peak area / corrected live time)

$\mu=$ attenuation coefficient

$x=$ attenuation foil thickness $(0.03 \mathrm{~cm})$ 
This approach considers all possible attenuations at any thickness of the foil. Also, the equations in Appendix A show that the independent areas and efficiencies for each energy were taken into consideration when performing the activity at E.O.I. calculation.

\section{Repeatability of Measurements:}

The attenuated and unattenuated resuits must show repeatability. To demonstrate repeatability, 5 measurements, using sample $2 \mathrm{~A}$, were taken consecutively on the same day. Two of the measurements were unattenuated and 3 of the measurements were attenuated. After each completed measurement, the sample was removed and then placed back on the detector like a new sampie. This was done to keep all variables consistent with how the original measurements were performed. Table 5 shows the corresponding standard deviation and percent error of the attenuated and unattenuated peak area and corrected live time.

\begin{tabular}{|c|c|c|c|c|c|c|c|}
\hline Niobium Foil Source & $\begin{array}{c}\text { Energy } \\
(\mathrm{keV})\end{array}$ & $\begin{array}{c}\text { Peak } \\
\text { Area }\end{array}$ & $\begin{array}{c}\text { Standard } \\
\text { Deviation }\end{array}$ & $\begin{array}{c}\text { Percent } \\
\text { Error }\end{array}$ & $\begin{array}{c}\text { Corrected Live } \\
\text { Time (sec) }\end{array}$ & $\begin{array}{c}\text { Standard } \\
\text { Deviation }\end{array}$ & $\begin{array}{c}\text { Percent } \\
\text { Error }\end{array}$ \\
\hline Linattenuated Source & 16.6 & 188657 & 427 & $0.226 \%$ & 776 & 0.707 & $0.0911 \%$ \\
Measurement 1 & 18.6 & 45419 & 294 & $0.648 \%$ & & 0.707 & $0.0911 \%$ \\
\hline Cnattenuated Source & 16.6 & 188053 & 427 & $0.227 \%$ & 777 & 0.707 & $0.0910 \%$ \\
Measurement 2 & 18.6 & 45835 & 294 & $0.642 \%$ & & 0.707 & $0.0910 \%$ \\
\hline Attenuated Source & 16.6 & 5281 & 288 & $5.45 \%$ & 788 & 0.577 & $0.0733 \%$ \\
Measurement 1 & 18.6 & 2578 & 97 & $3.75 \%$ & & 0.577 & $0.0733 \%$ \\
\hline Attenuated Source & 16.6 & 4756 & 288 & $6.06 \%$ & 788 & 0.577 & $0.0733 \%$ \\
Measurement 2 & 18.6 & 2704 & 97 & $3.58 \%$ & & 0.577 & $0.0733 \%$ \\
\hline Attenuated Source & 16.6 & 5224 & 288 & $5.51 \%$ & 788 & 0.577 & $0.0733 \%$ \\
Measurement 3 & 18.6 & 2514 & 97 & $3.85 \%$ & & 0.577 & $0.0733 \%$ \\
\hline
\end{tabular}

Table 5: Standard Deviation and Percent Error of counts per second for Repeated Measurements

The percent error for the attenuated peak area was approximately $6 \%$ for the $\mathrm{K}$-alpha $\mathrm{x}$-ray and $4 \%$ for the K-beta $x$-ray. The unattenuated counts per second had an error of less than $1 \%$. These results demonstrate the repeatability of the measurements for the unattenuated source. They also show that the attenuated source measurements were repeatable to within approximately $6 \%$ error. The corrected live time had less than $1 \%$ error for both the attenuated and unattenuated source. Error Analysis:

The possibility of error was apparent in all areas of this experiment. The error most apparent in the efficiency curve calculation was due to uncertainities in the calibration source. The 
most important of these uncertainties was the Am-241 (HP-199) source. This source had an error of $4 \%$ in it's activity according to the certificate of calibration. The maximum uncertainty for the mixed gamma source (HP-5) was $5 \%$ according to it's certificate of calibration. From this information, it was concluded that the efficiency curve had an approximate $5 \%$ error.

The measurement of peak areas had an approximate error of $1 \%$ for an unattenuated source. This error was observed when determining if the tape used to tape the foil to the sample cards had any affect to the results returned. It was also observed when doing the repeatability measurements. This shows the reproducibility of the results were within $1 \%$. Although when the source was attenuated by a piece of niobium foil, the peak area had an approximate reproduction error of $6 \%$. This error was seen when trying to show the reproducibility of the measurements.

The corrected live time had an estimated error of $1 \%$ as seen when demonstrating reproducibility. The error due to mass was approximately $0.1 \%$. This was because the mass was measured to within a tenth of a milligram on the 5 -place balance. The $\mathrm{K} \mathrm{x}$-ray emission probability $\left(0.115 \pm 0.003\right.$ per decay) had an error of $3 \% .^{2}$ The half-life of Nb-93m $(16.13 \pm 0.15$ years $)$ had an error of $0.9 \% .^{2}$

The largest source of error was probably the attenuation coefficient. The attenuated and unattenuated counts per second have an approximate $6 \%$ and $1 \%$ error from the peak. This means the error for the attenuation coefficient was approximately $7 \%$. The following table lists these errors.

\begin{tabular}{|c|c|}
\hline Name of Error & ERROR \\
\hline Efficiency & $5 \%$ \\
\hline Peak Area (unattenuated / attenuated) & $1 \% / 6 \%$ \\
\hline Corrected Live Time & $1 \%$ \\
\hline Mass & $0.1 \%$ \\
\hline K x-ray Emission & $3 \%$ \\
\hline Half-Life of Nb-93m & $0.9 \%$ \\
\hline Attenuation Coefficient & $7 \%$ \\
\hline
\end{tabular}

Table 6: Estimates of Error

Errors are additive when the factors in the equation are being multiplied or divided. The greater error is taken when adding terms. This means that the activity shown in Appendix A should have a total uncertainity of about $20 \%$. 
SUMMARY AND CONCLUSION

The activity of $\mathrm{Nb}-93 \mathrm{~m}$ in the niobium foils found in Appendix A, was determined by the use of a Gamma-X coaxial detector. The detector was calibrated by a calibrated Am- 241 point source (HP-199) and a calibrated mixed gamma source (HP-5). The calibration information, found in table 2, produced the calibration curve (see figure 1). The niobium foils were then weighed and mounted at the center of sample cards with tape; similar to how the calibration sources were mounted. The tape used to mount the samples, was verified not to attenuate the foil. Each sample was then counted for 15 minutes, 1 inch from the face of the detector. The same position the calibration sources used.

Then an attenuation coefficient was determined by counting sample $2 \mathrm{~A}$ with and without an attenuation foil $(0.03 \mathrm{~cm}$ thick). The coefficient was used to determine the amount of attenuated radiation through the source foil to correct the activity. After completing these measurements, two more possible data corrections were addressed. The possibility of Ta-182 induced fluorescence $x$ rays causing interference and the content of Tantalum in the Niobium foil before irradiation. Through measurement, the amount of Ta-182 activity was determined to be less than $2 \%$; therefore, no correction for Ta-182 induced fluorescence $\mathrm{x}$-rays was needed. The chemical analysis information provided showed that the Tantalum content was less than $5 \mathrm{ppm}$; therefore, no correction was needed.

The computer software automatic determination of peak areas for the low-energy peaks had a problem. The use of a manual interactive peak search was required. The corrected peak areas are contained in Appendix A.

The problem of repeatability was also addressed. To demonstrate the repeatability of the results. 5 measurements of foil sample $2 \mathrm{~A}$ were performed consecutively on the same day. Each measurement was treated like a measurement of a new sample. This was done to keep the results consistent with how the original measurements were performed. Two of the measurements were unattenuated and three of the measurements were attenuated. As seen in table 5, the peak area of the unattenuated measurements had an error of less than $1 \%$, while the attenuated measurements had an error of approximately $6 \%$ for the K-alpha x-ray. The corrected live time for both the attenuated and 
unattenuated measurements had an error of less than $1 \%$. This indicates that the results were

repeatable. Although, the larger attenuated measurement error might be reduced by counting the foil for more than 15 minutes or until the peak area was around 100,000 .

The estimate of total uncertainty was performed to show the accuracy of the activity at E.O.I. Table 6 shows the error for the various measured and given values. A conservative approach was taken when estimating the error of the various values. This makes the total uncertainty an overly conservative estimate.

The information contained in Appendix A is considered to be as accurate as reasonable. The equations contained in Appendix A show that the independent areas and efficiencies for each $\mathrm{x}$ ray energy are taken into consideration when performing the calculation of activity at the E.O.I. It is felt that these results are best results that can be found by the detection system used. 


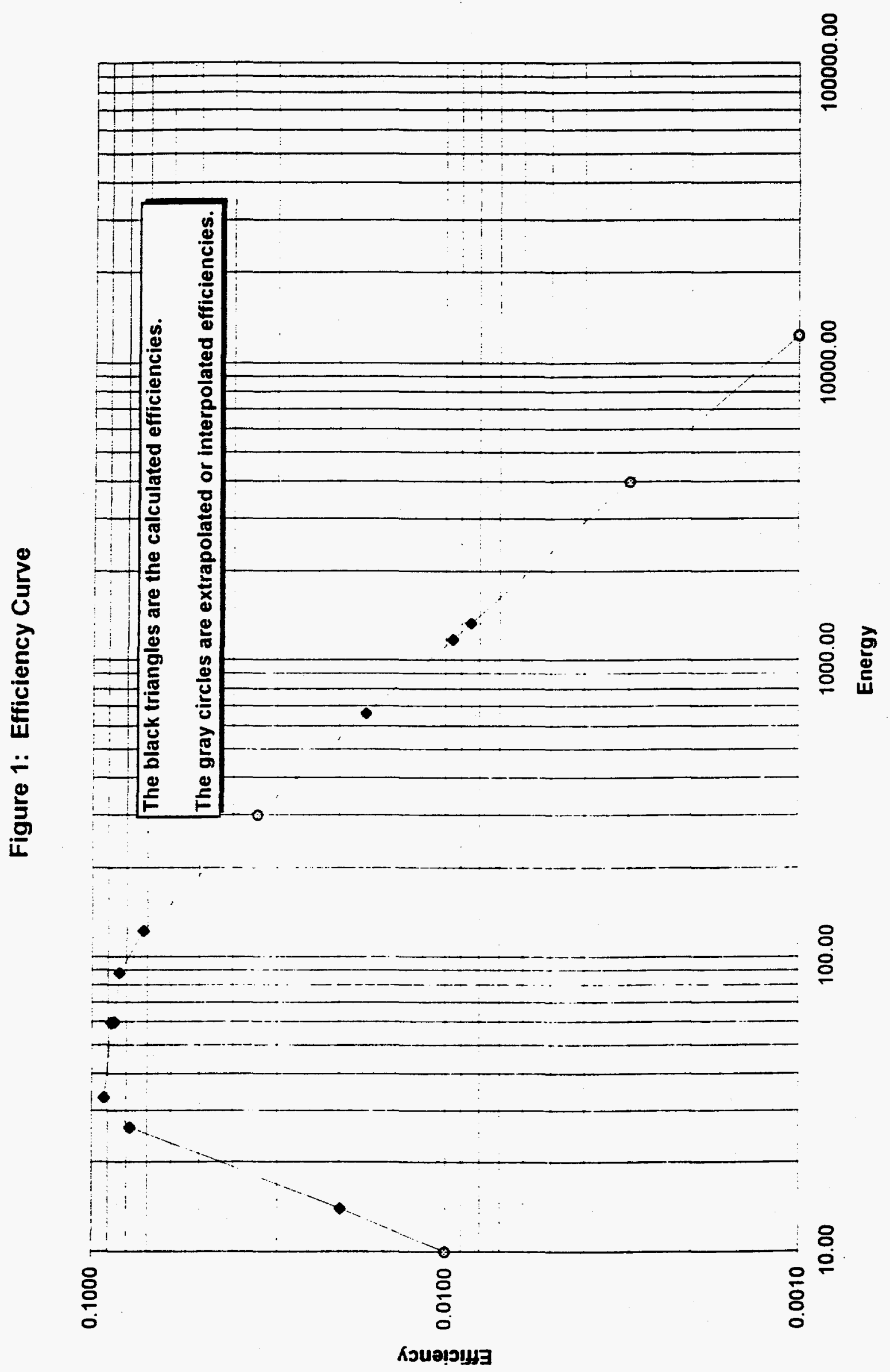


Appendix A

Activities measured

in

Niobium Foils 


\begin{tabular}{|c|c|c|c|c|c|c|c|}
\hline Sample & $\begin{array}{c}\text { Energy } \\
\text { (keV) }\end{array}$ & Efficiency & Peak Area & $\begin{array}{l}\text { Corrected Live } \\
\text { Time (sec) }\end{array}$ & $\begin{array}{l}\text { Mass } \\
\text { (mg) }\end{array}$ & $\begin{array}{c}\text { E.O.I. Activity } \\
(\mu \mathrm{C} / \mathrm{mg})\end{array}$ & $\begin{array}{l}\text { Estimated } \\
\% \text { Error }\end{array}$ \\
\hline \multirow[t]{2}{*}{$\overline{I A}$} & 16.6 & 0.0229 & 74452 & & & & \\
\hline & 18.6 & 0.0257 & 18194 & 854 & 16.80 & 0.252 & $20 \%$ \\
\hline \multirow[t]{2}{*}{ 1B } & 16.6 & 0.0229 & 73202 & & & & \\
\hline & 18.6 & 0.0257 & 18487 & 852 & 17.04 & 0.246 & $20 \%$ \\
\hline \multirow[t]{2}{*}{$\overline{I C}$} & 16.6 & 0.0229 & 69560 & & & & \\
\hline & 18.6 & 0.0257 & 18971 & 854 & 17.08 & 0.236 & $20 \%$ \\
\hline \multirow[t]{2}{*}{$2 A$} & 16.6 & 0.0229 & 185966 & & & & \\
\hline & 18.6 & 0.0257 & 45104 & 728 & 16.88 & 0.734 & $20 \%$ \\
\hline \multirow[t]{2}{*}{$3 \mathrm{~A}$} & 16.6 & 0.0229 & 110704 & & & & \\
\hline & 18.6 & 0.0257 & 26848 & 819 & 17.42 & 0.376 & $20 \%$ \\
\hline \multirow[t]{2}{*}{$3 \mathrm{~B}$} & 16.6 & 0.0229 & 110802 & & & & \\
\hline & 18.6 & 0.0257 & 27144 & 819 & 14.28 & 0.460 & $20 \%$ \\
\hline \multirow[t]{2}{*}{$3 \mathrm{C}$} & 16.6 & 0.0229 & 112841 & & & & \\
\hline & 18.6 & 0.0257 & 27603 & 818 & 16.85 & 0.398 & $20 \%$ \\
\hline \multirow[t]{2}{*}{$\overline{4 A}$} & 16.6 & 0.0229 & 54910 & & & & \\
\hline & 18.6 & 0.0257 & 14901 & 840 & 17.30 & 0.187 & $20 \%$ \\
\hline \multirow[t]{2}{*}{$4 \mathrm{~B}$} & 16.6 & 0.0229 & 58587 & & & & \\
\hline & 18.6 & 0.0257 & 14864 & 829 & 17.33 & 0.199 & $20 \%$ \\
\hline \multirow[t]{2}{*}{$\overline{4 C}$} & 16.6 & 0.0229 & 57501 & & & & \\
\hline & 18.6 & 0.0257 & 14687 & 843 & 16.73 & 0.199 & $20 \%$ \\
\hline \multirow[t]{2}{*}{$5 \mathrm{~A}$} & 16.6 & 0.0229 & 138254 & & & & \\
\hline & 18.6 & 0.0257 & 33798 & 806 & 16.97 & 0.491 & $20 \%$ \\
\hline \multirow[t]{2}{*}{$5 B$} & 16.6 & 0.0229 & 137468 & & & & \\
\hline & 18.6 & 0.0257 & 33656 & 808 & 16.79 & 0.492 & $20 \%$ \\
\hline \multirow[t]{2}{*}{$5 C$} & 16.6 & 0.0229 & 137152 & & & & \\
\hline & 18.6 & 0.0257 & 33702 & 807 & 16.94 & 0.488 & $20 \%$ \\
\hline \multirow[t]{2}{*}{$6 \mathrm{~A}$} & $\overline{16.6}$ & 0.0229 & 99268 & & & & \\
\hline & 18.6 & 0.0257 & 24208 & 832 & 16.93 & 0.342 & $20 \%$ \\
\hline \multirow[t]{2}{*}{$6 \mathrm{~B}$} & 16.6 & 0.0229 & 99418 & & & & \\
\hline & 18.6 & 0.0257 & 24110 & 832 & 16.91 & 0.343 & $20 \%$ \\
\hline \multirow[t]{2}{*}{$6 \mathrm{C}$} & 16.6 & 0.0229 & 96401 & & & & \\
\hline & 18.6 & 0.0257 & 23597 & 835 & 16.75 & 0.335 & $20 \%$ \\
\hline
\end{tabular}

* Efficiencies are from table 1

Activity at E.O.I. $=\mathrm{e}^{\lambda t} \cdot \frac{\text { decays }}{\sec } \cdot \frac{1}{0.115} \cdot \frac{1}{\text { mass }} \cdot \frac{1}{37000}$

where:

$\frac{\text { decays }}{\sec }=\left[\left(\frac{\text { peak area }}{\varepsilon_{\mathrm{K}_{\mathrm{x}}}} \cdot \frac{\mu_{\mathrm{K}_{\mathrm{z}}} \cdot \mathrm{x}}{\left(1-\mathrm{e}^{-\mu_{\mathrm{K}_{\mathrm{\alpha}}} \cdot \mathrm{x}}\right)}\right)+\left(\frac{\text { peak area }}{\varepsilon_{\mathrm{K}_{\mathrm{\beta}}}} \cdot \frac{\mu_{\mathrm{K}_{\mathrm{\beta}}} \cdot \mathrm{x}}{\left(1-\mathrm{e}^{-\mu_{\mathrm{K}_{\mathrm{\beta}}} \cdot \mathrm{x}}\right)}\right)\right] \cdot \frac{1}{\text { corrected live time }}$

and

$\lambda=\ln (2) /$ half life (half life $=16.13$ years)

$\mathrm{t}=$ decay time since end of irradiation (E.O.I.) \{778 days $\}$

$\varepsilon=$ efficiency $\left(K_{\alpha}\right.$ is the $16.6 \mathrm{keV}$ and $\mathrm{K}_{\beta}$ is the $18.6 \mathrm{keV} \mathrm{x}$-ray)

$\mathrm{x}=$ thickness of attenuation foil $(0.3 \mathrm{~mm})$ 


\section{REFERENCES:}

1. Knoll, Glenn F., Radiation Detection and Measurement, John Wiley and Sons, Inc., New York, 1989.

2. ASTM - E 1297 - 89, Measuring Fast-Neutron Reaction Rates by Radioactivation of Niobium, 1990 ASTM Annual Book of Standards. 


\section{APPENDIX II}

Figures for Beam History, Differential Neutron Flux, and Neutron Energy Flux 


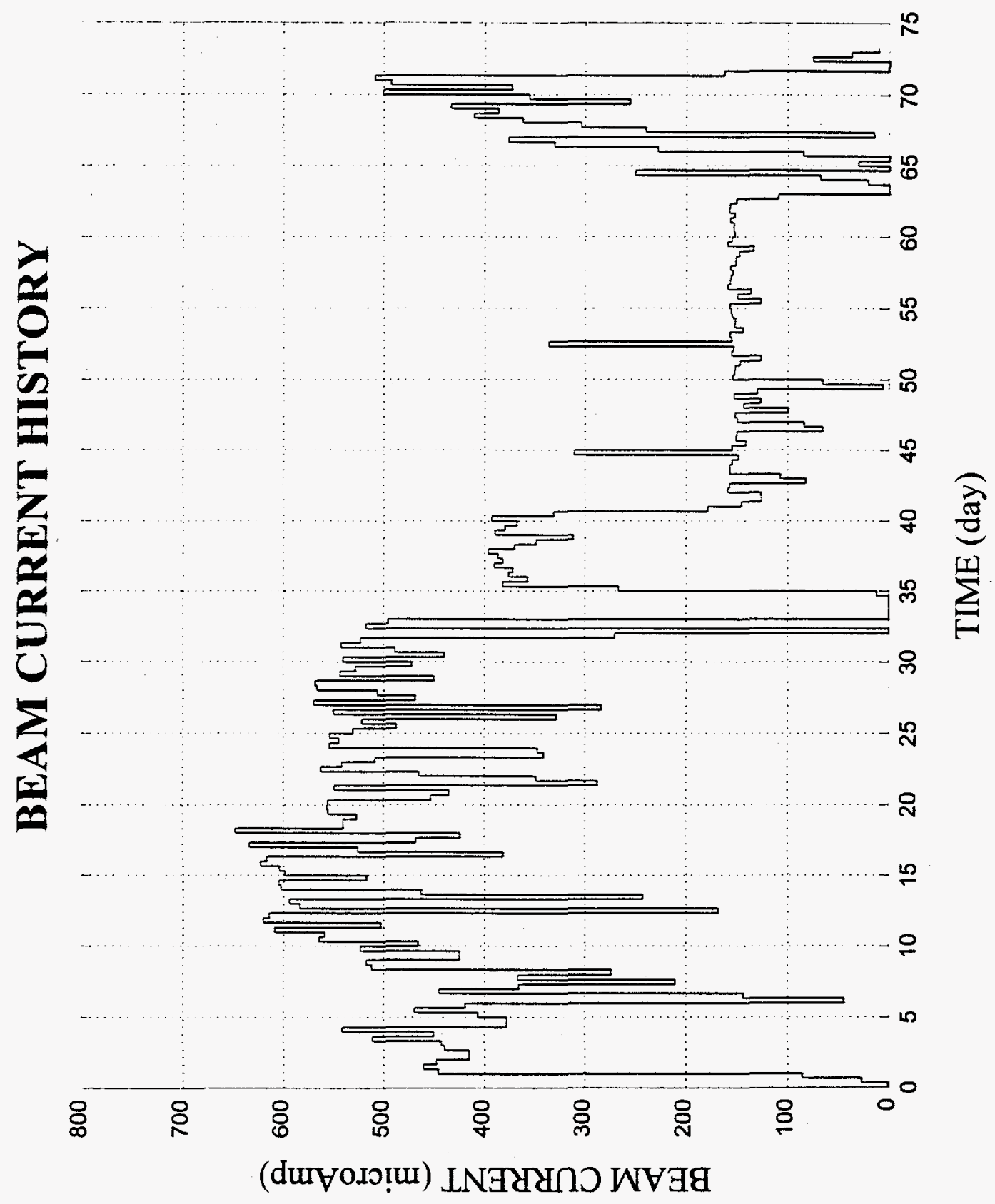




\section{1-1 TO 1-5 DIFFERENTIAL NEUTRON FLUX}

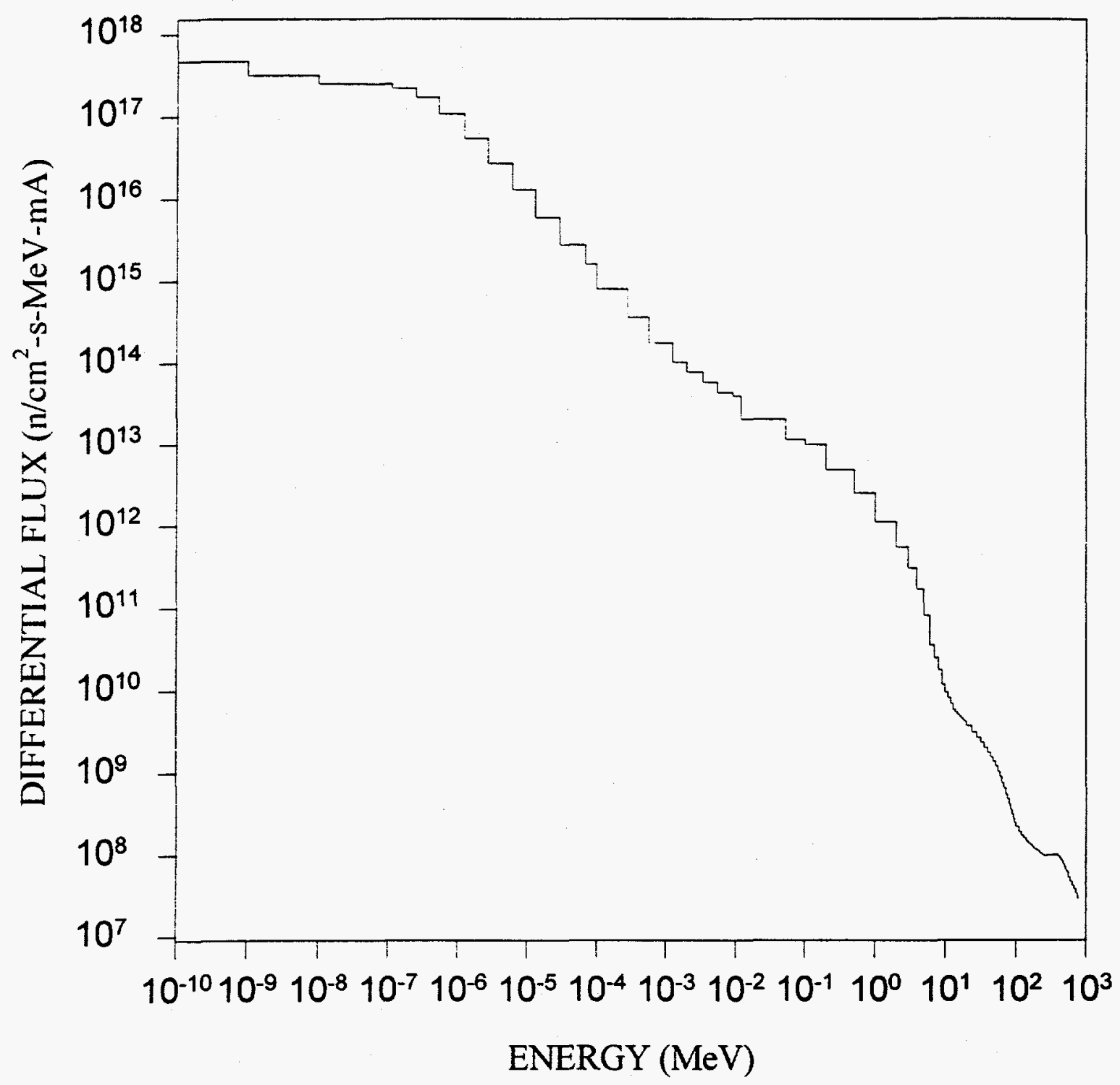




\section{1-1 TO 1-5 NEUTRON FLUX}

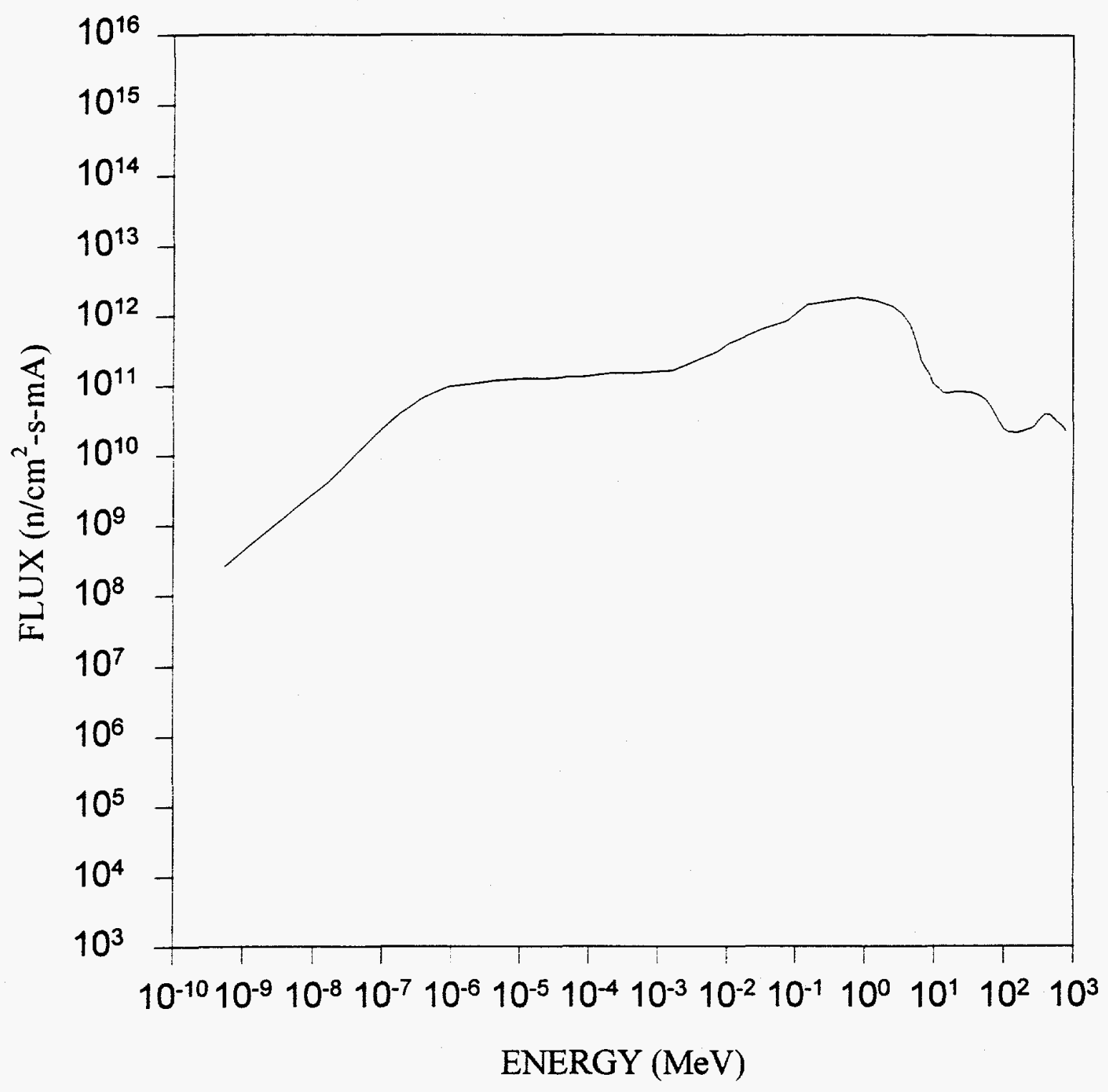




\section{2-1 TO 2-5 DIFFERENTIAL NEUTRON FLUX}

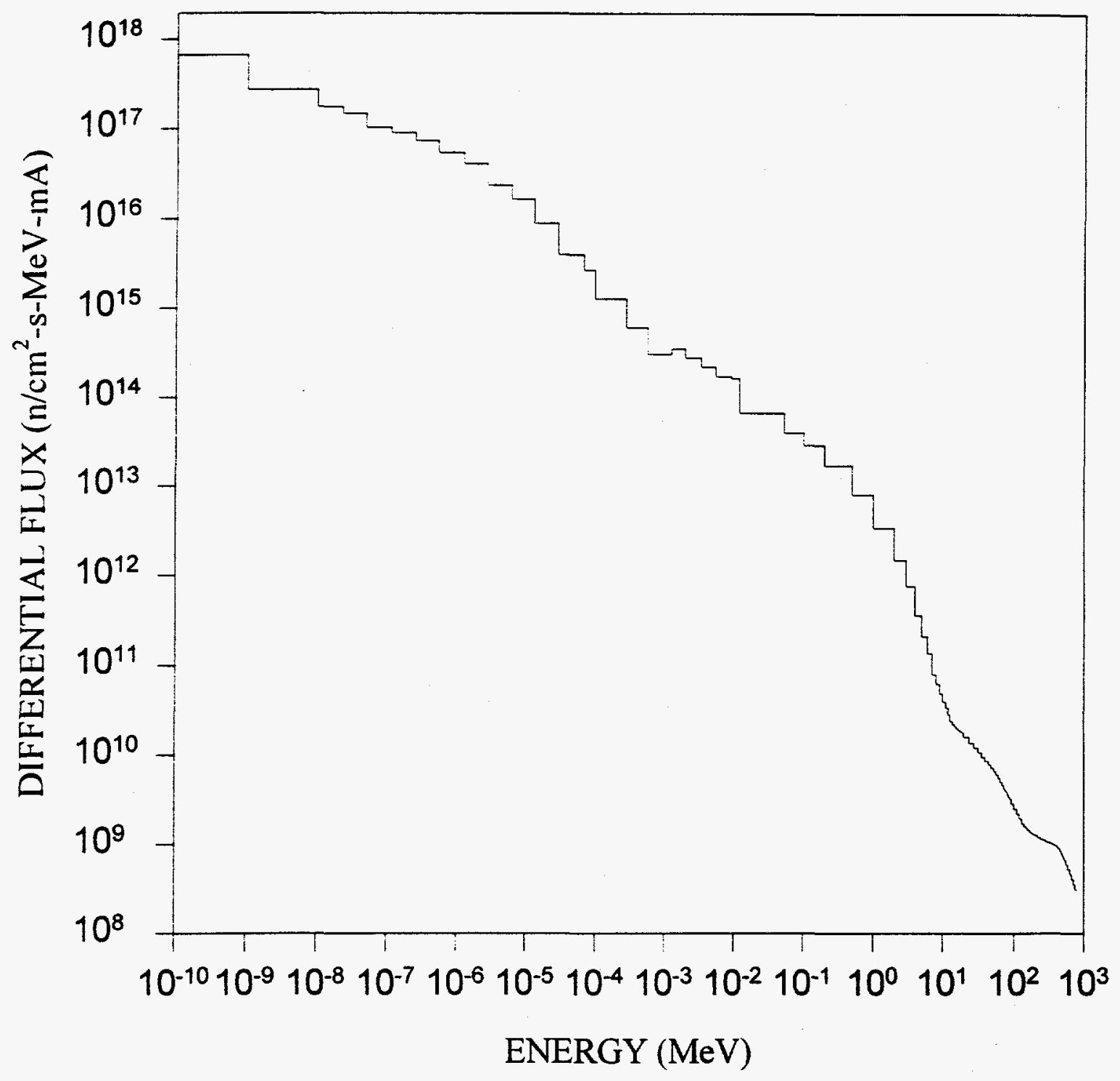




\section{2-1 TO 2-5 NEUTRON FLUX}

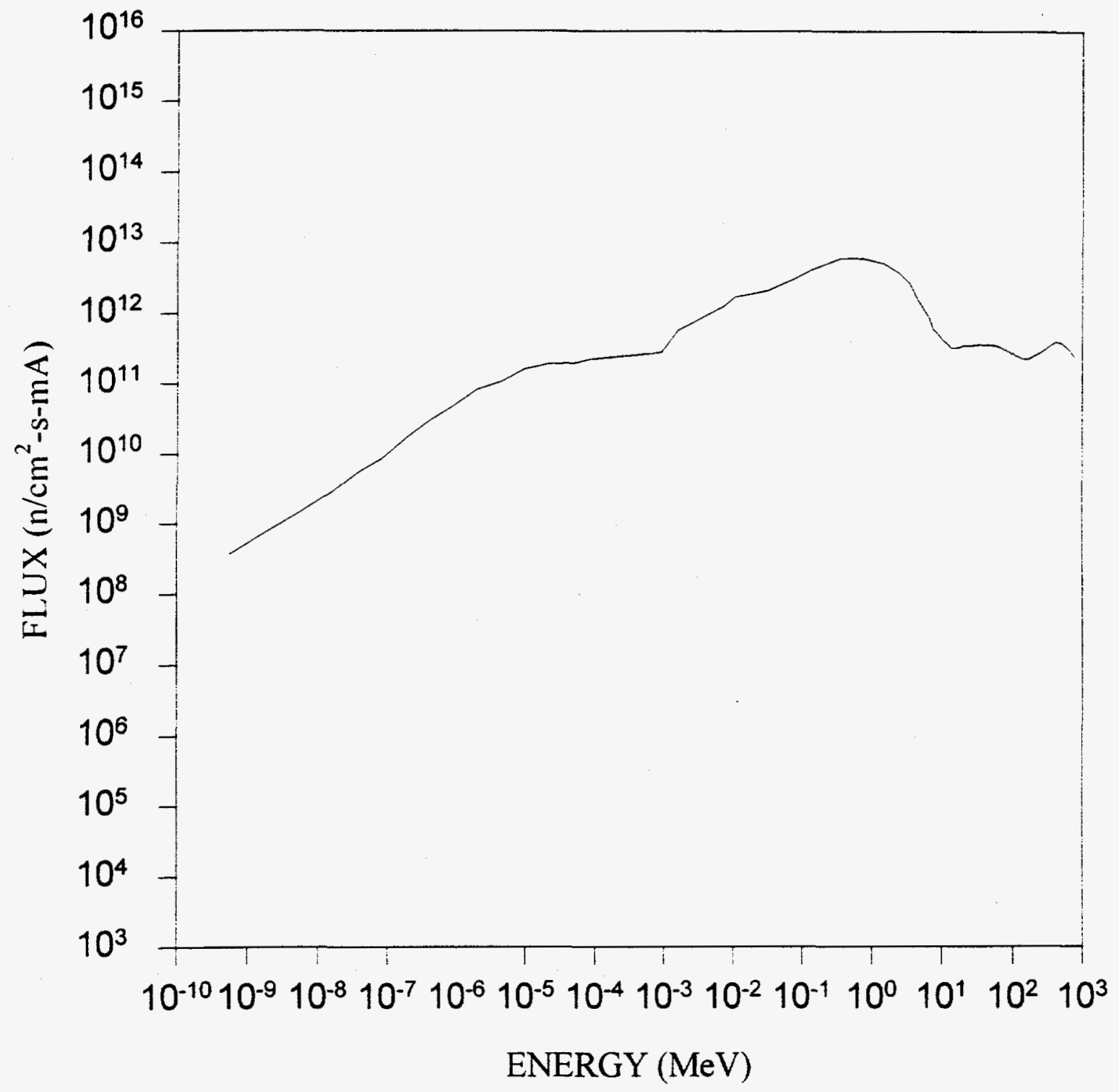




\section{3-1 TO 3-5 DIFFERENTIAL NEUTRON FLUX}

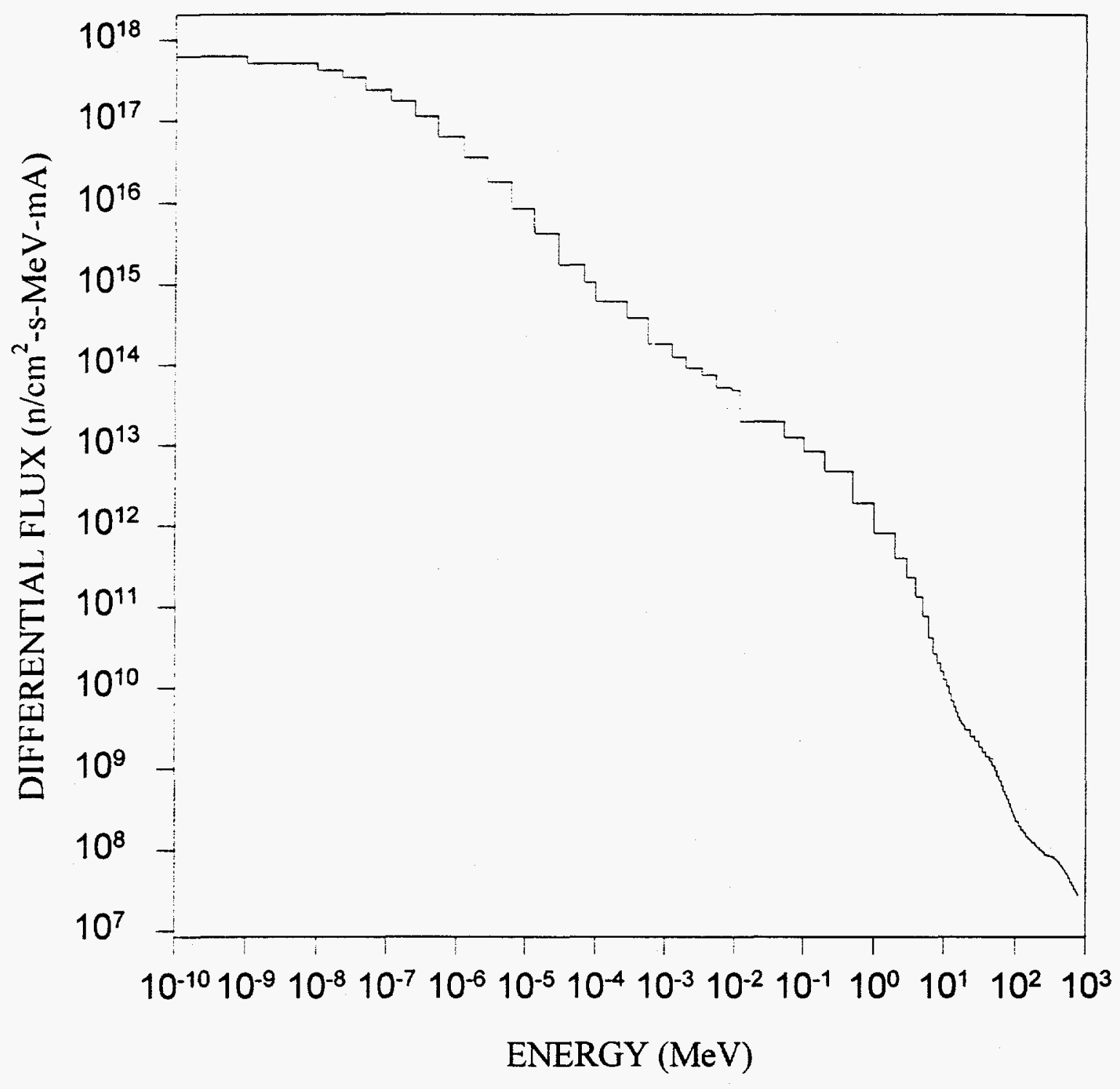




\section{3-1 TO 3-5 NEUTRON FLUX}

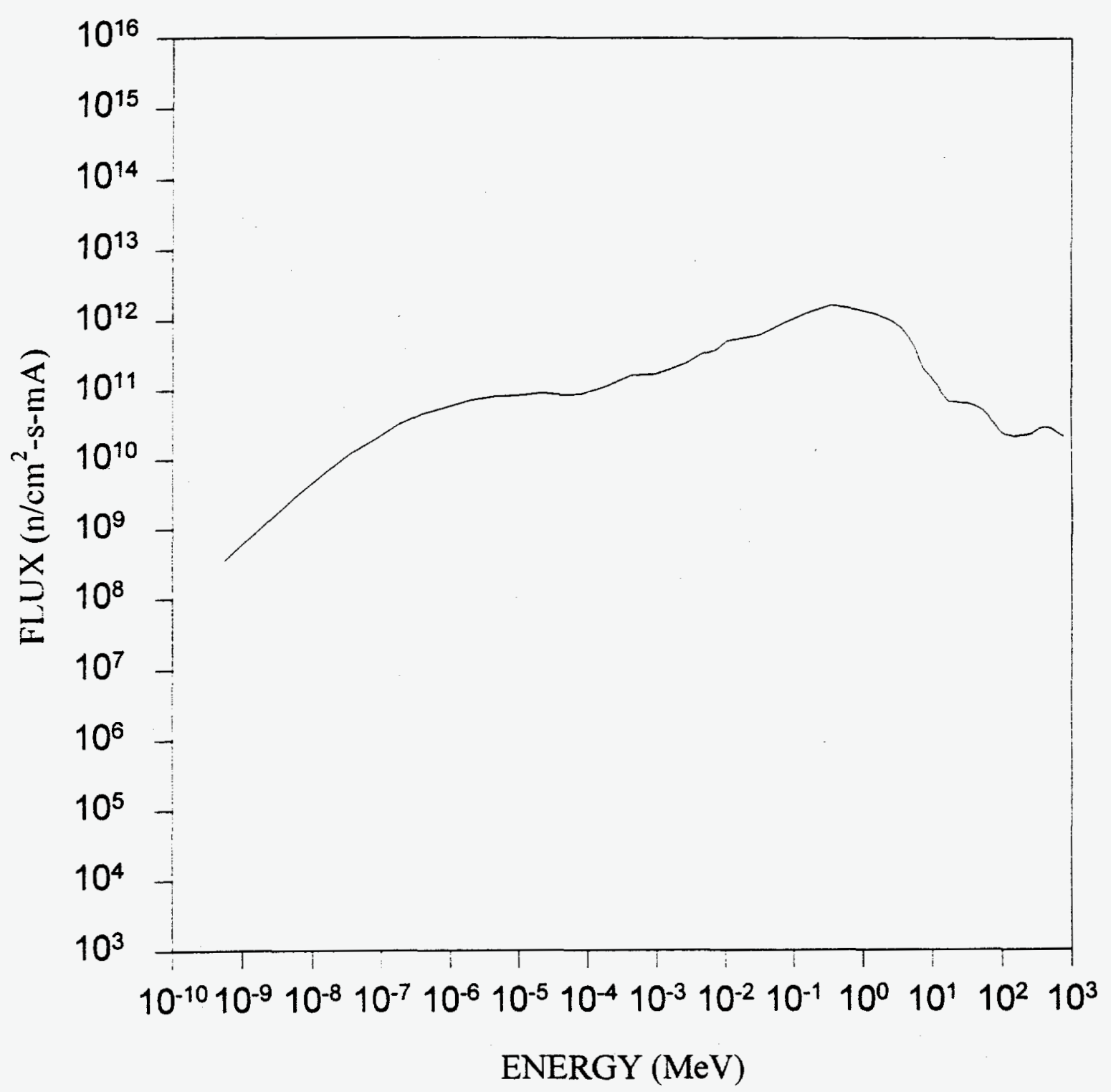




\section{4-1 TO 4-5 DIFFERENTIAL NEUTRON FLUX}

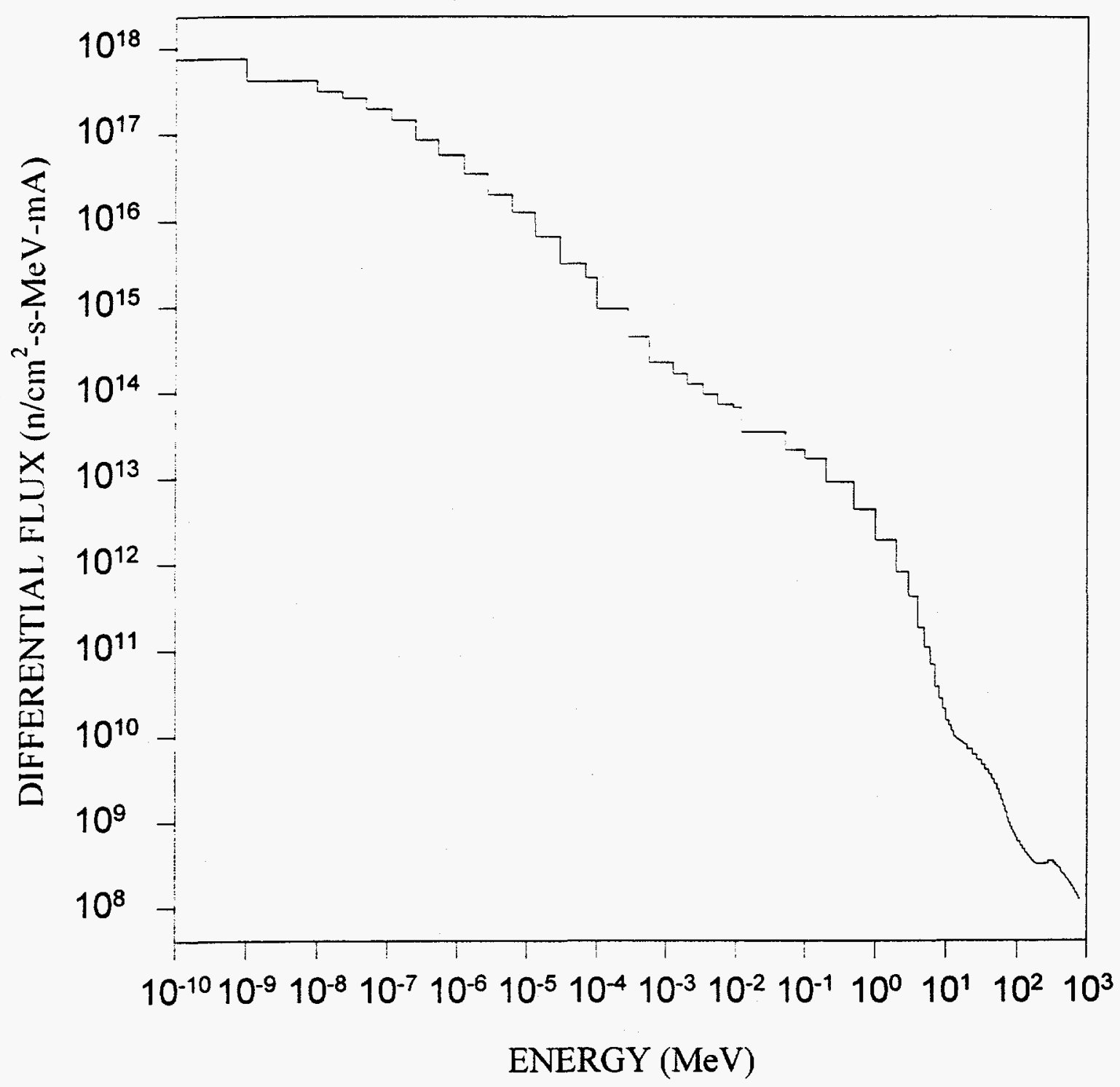




\section{4-1 TO 4-5 NEUTRON FLUX}

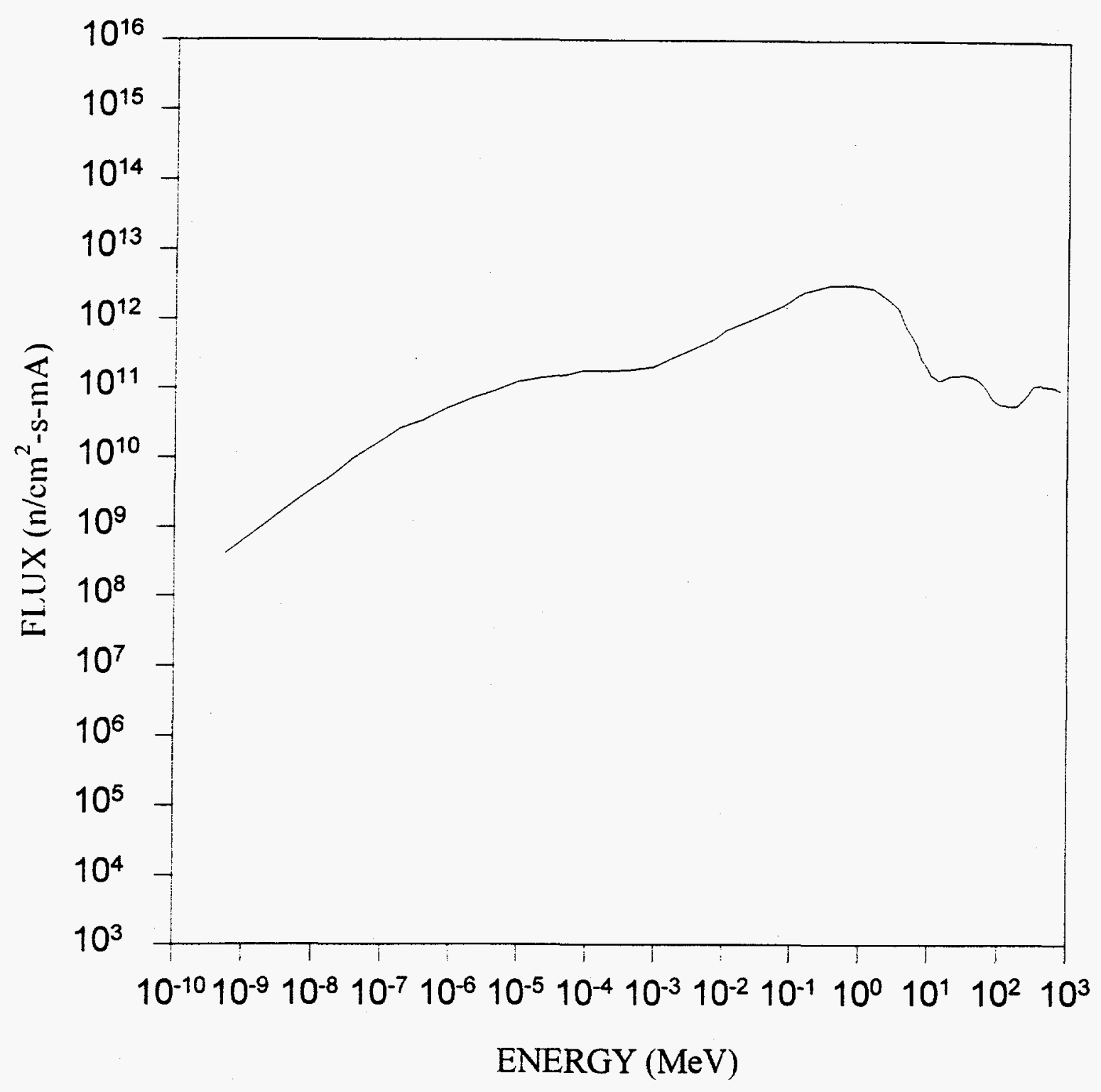


5-1 TO 5-5 DIFFERENTIAL NEUTRON FLUX

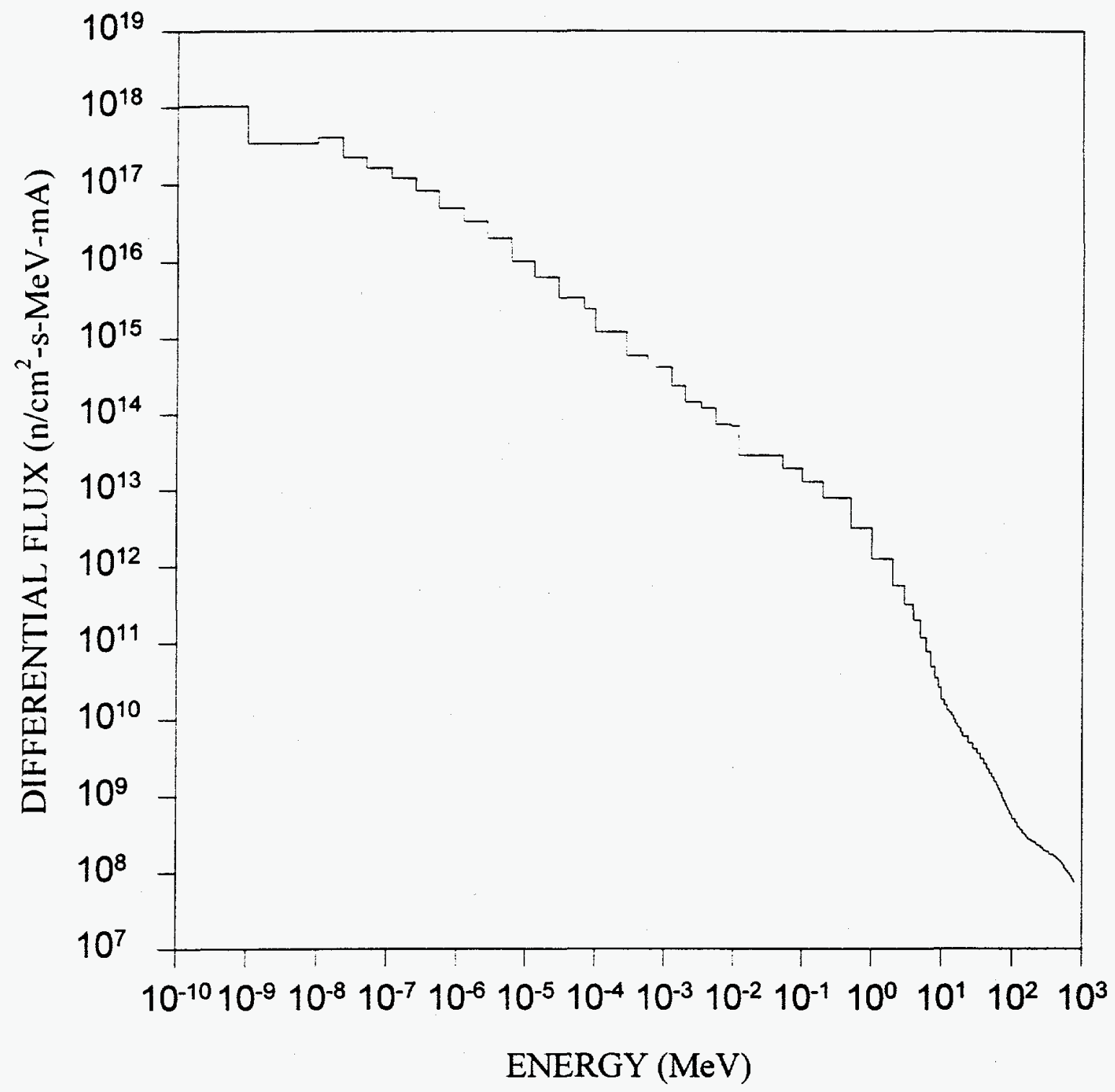


5-1 TO 5-5 NEUTRON FLUX

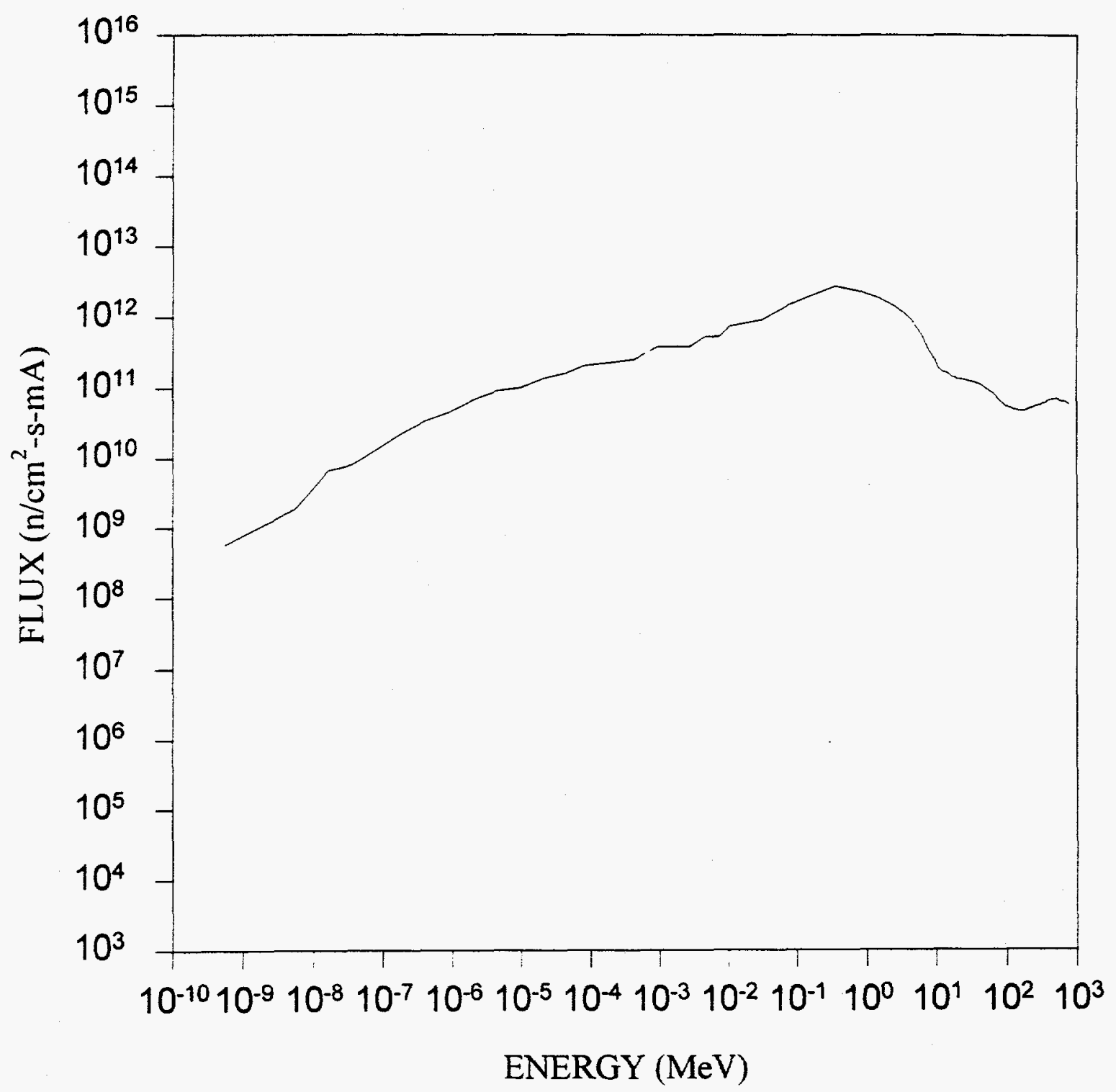




\section{6-1 TO 6-5 DIFFERENTIAL NEUTRON FLUX}

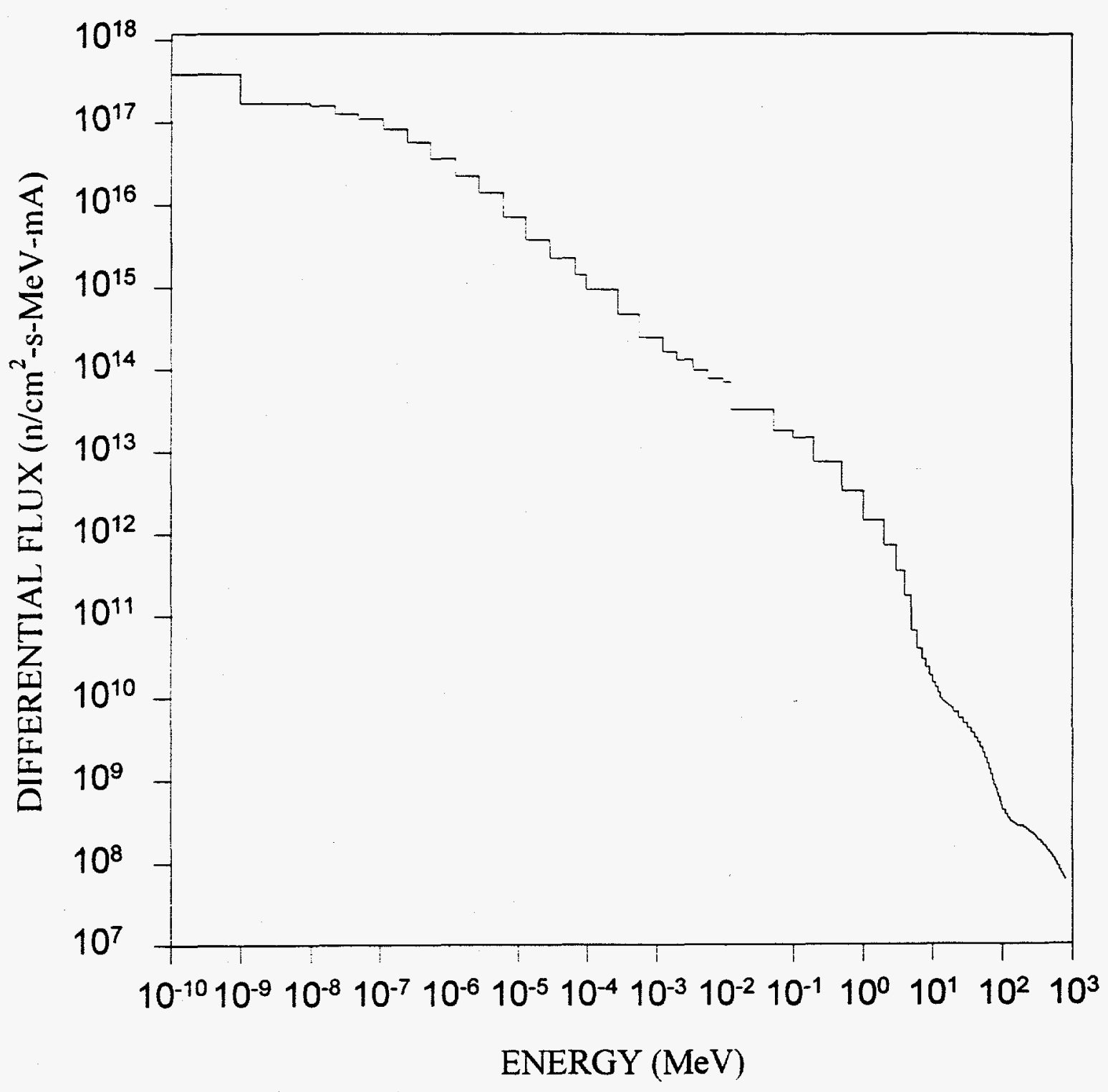




\section{6-1 TO 6-5 NEUTRON FLUX}

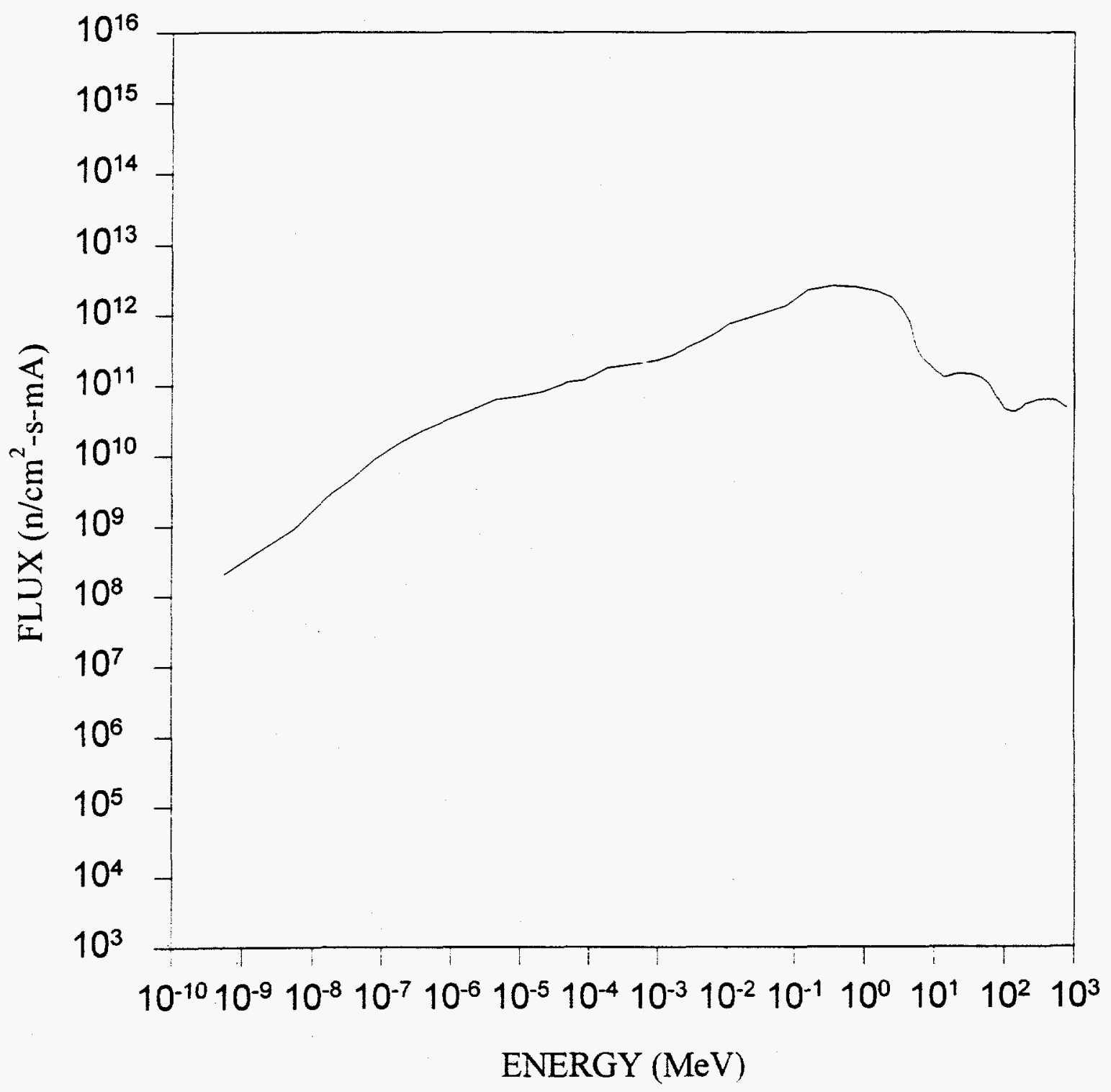




\section{APPENDIX III}

Reactions, Corrected Saturated Activities, and Integrated Group Energy Fluxes and Fluences 


\section{SUMMARY CALCULATIONS}

FOR 1-1 TO 1-5

\begin{tabular}{|c|c|c|c|c|c|c|c|c|c|c|}
\hline \multicolumn{11}{|c|}{ DOSIMETRY ACTIVITIES } \\
\hline & MEASURED & $+1-8$ & BEFORE & DIF\& & AFTER & DIF\& & $\mathrm{CHI}$ & REACTION & 908 & LIMITS \\
\hline 1 & $4.27 E+11$ & 10.0 & $4.89 E+11$ & -14.6 & $4.71 E+11$ & -10.5 & .7 & $\Xi E 54(\mathrm{~N}, *) \mathrm{MN} 54$ & $2.00 \mathrm{E}+00$ & $3.80 E+02$ \\
\hline 2 & $2.58 E+11$ & 10.0 & $2.44 E+11$ & 5.6 & $2.45 E+11$ & 5.2 & .2 & ZE58 (N, G)FE59 & $1.00 \mathrm{E}-08$ & $5.25 \mathrm{E}-02$ \\
\hline 3 & $5.72 E+10$ & 10.0 & $5.35 E+10$ & 5.5 & $5.33 E+10$ & 5.8 & .2 & $\operatorname{Co5} 9(N, 2 N) \operatorname{Co5} 8$ & $1.20 \mathrm{E}+01$ & $3.60 E+01$ \\
\hline 4 & $1.85 E+10$ & 10.0 & $1.42 \mathrm{E}+10$ & 23.3 & $1.45 E+10$ & 21.7 & .6 & $\cos 9(N, 3 N) \cos 7$ & $2.00 \mathrm{E}+01$ & $5.20 \mathrm{E}+01$ \\
\hline 5 & $7.15 E+12$ & 15.0 & $8.12 E+12$ & -13.5 & $8.21 E+12$ & -14.8 & .2 & $\operatorname{Co59}(\mathrm{N}, \mathrm{G}) \mathrm{CO} 60$ & $1.00 \mathrm{E}-08$ & $1.00 E-04$ \\
\hline 6 & $1.55 E+10$ & 15.0 & $1.77 E+10$ & -14.5 & $1.67 E+10$ & -7.5 & .3 & $\because: I 60(N, P) C 060$ & $5.00 E+00$ & $2.80 \mathrm{E}+01$ \\
\hline 7 & $1.36 E+09$ & 10.0 & $1.08 \mathrm{E}+09$ & 20.6 & $1.14 E+09$ & 16.4 & 1.0 & $C U(N, *) 54 M N$ & $6.80 E+01$ & $6.80 E+02$ \\
\hline 8 & $4.67 E+08$ & 25.0 & $8.82 E+07$ & 81.1 & $9.19 E+07$ & 80.3 & 10.2 & $C U\left(N,{ }^{*}\right) 59 F E$ & $5.60 \mathrm{E}+01$ & $6.80 E+02$ \\
\hline 9 & $7.04 E+08$ & 10.0 & $8.03 E+08$ & -14.0 & $8.22 E+08$ & -16.7 & .5 & $\mathrm{CU}(\mathrm{N}, *) 56 \mathrm{CO}$ & $6.00 E+01$ & $6.40 E+02$ \\
\hline 10 & $3.83 E+09$ & 10.0 & $4.16 \mathrm{E}+09$ & -8.7 & $4.23 E+09$ & -10.5 & .4 & $\theta(N, *) 57 C 0$ & $4.00 E+01$ & $5.60 E+02$ \\
\hline 11 & $6.99 \mathrm{E}+09$ & 10.0 & $6.61 E+09$ & 5.5 & $6.73 E+09$ & 3.3 & $\therefore$ & $z(\mathrm{~N}, *) 58 \mathrm{CO}$ & $2.80 E+01$ & $5.20 E+02$ \\
\hline 12 & $5.25 E+09$ & 25.0 & $1.22 E+09$ & 76.7 & $1.25 E+09$ & 76.2 & 9.3 & $\mathrm{CU}(\mathrm{N}, *) 60 \mathrm{CO}$ & $3.20 E+01$ & $6.00 E+02$ \\
\hline 13 & $5.45 E+11$ & 20.0 & $5.41 E+11$ & .6 & $5.07 E+11$ & 7.0 & .0 & $\operatorname{SB9} 3\left(\mathrm{~N}, \mathrm{~N}^{\prime}\right) \mathrm{NB} 93 \mathrm{M}$ & $5.00 \mathrm{E}-01$ & $5.00 \mathrm{E}+00$ \\
\hline
\end{tabular}

STD. DEV. $=34.6634 .05$

CHISQ $=37.28 \quad 34.22$

SUMMARY OF INTEGRATED GROUP ENERGY FUUXES AND FLUENCES

\begin{tabular}{|c|c|c|c|c|c|}
\hline $\begin{array}{c}\text { ENERGY } \\
\text { MeV }\end{array}$ & $\begin{array}{c}\text { FLUX } \\
\mathrm{n} / \mathrm{cm} 2-\mathrm{s}-\mathrm{mA}\end{array}$ & $\begin{array}{l}\text { FLUX } \\
\mathrm{n} / \mathrm{cm} 2-\mathrm{s}\end{array}$ & $\begin{array}{r}\text { EUUENCE } \\
n / \mathrm{cm} 2\end{array}$ & \multicolumn{2}{|c|}{ DEVIATION } \\
\hline$<1.0$ & $2.836 \mathrm{E}+12$ & $8.712 E+11$ & $5.520 \mathrm{E}+18$ & $+1-8.22$ & 32.98 \\
\hline $0.1-1.0$ & $3.451 E+12$ & $1.060 \mathrm{E}+12$ & 6. $: 28 \mathrm{E}+18$ & $+1-5.46$ & 40.14 \\
\hline $1.0-10.0$ & $2.096 \mathrm{E}+12$ & $6.438 \mathrm{E}+11$ & $4.379 E+18$ & $+1-8.90$ & 24.37 \\
\hline $10.0-20.0$ & $5.524 E+10$ & $1.694 \mathrm{E}+10$ & $1.573 E+17$ & $+/-11.21$ & .64 \\
\hline $20.0-40.0$ & $5.548 \mathrm{E}+10$ & $1.704 \mathrm{E}+10$ & 1. $280 E+17$ & $+/-12.12$ & .65 \\
\hline $40.0-100.0$ & $4.596 \mathrm{E}+10$ & $1.412 \mathrm{E}+10$ & $8.947 E+16$ & $+/-15.94$ & .53 \\
\hline $100.0-200.0$ & $1.396 \mathrm{E}+10$ & $4.289 \mathrm{E}+09$ & $2.77 E+16$ & $+1-23.59$ & .16 \\
\hline$>200.0$ & $4.107 E+10$ & $1.262 \mathrm{E}+10$ & $7.594 \mathrm{E}+16$ & $+1-22.22$ & .48 \\
\hline TOTAL & $8.598 \mathrm{E}+12$ & $2.641 \mathrm{E}+12$ & 1. $574 \mathrm{E}+19$ & $+1-6.51$ & \\
\hline
\end{tabular}

THE PERCENTAGE OF NEUTRONS ABOVE $20 \mathrm{YeV}$ IS -1.82 


\section{SUMMARY CALCULATIONS}

FOR 2-1 TO 2-5

\begin{tabular}{|c|c|c|c|c|c|c|c|c|c|c|}
\hline & & & & & NOS IIRE & $R Y A C$ & ITIE & & & \\
\hline & MEASURED & $+1-8$ & 3EFORE & DIF 8 & AFTER & DIF 8 & $\mathrm{CHI}$ & EEACTION & 908 & LIMITS \\
\hline$i$ & $1.29 E+12$ & 15.0 & $9.92 E+11$ & 23.1 & $9.78 E+11$ & 24.2 & 1.7 & 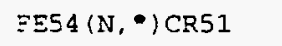 & $2.80 E+01$ & $6.40 E+02$ \\
\hline 2 & $2.01 E+12$ & 10.0 & $2.26 E+12$ & -12.4 & $2.23 E+12$ & -11.2 & .5 & EE54 (N, *)MN54 & $2.00 E+00$ & $5.20 E+02$ \\
\hline 3 & $4.98 E+11$ & 10.0 & $4.27 E+11$ & 14.4 & $4.66 \mathrm{E}+11$ & 6.5 & .6 & EE58 (N, G)FE59 & $5.00 E-08$ & $2.00 E-01$ \\
\hline 4 & $2.42 \Xi+11$ & 10.0 & $2.37 E+11$ & 2.3 & $2.31 E+11$ & 4.6 & .0 & $\cos 9(N, 2 N) \cos 8$ & $1.20 E+01$ & $4.00 \mathrm{E}+01$ \\
\hline 5. & $9.00 \mathrm{E}+10$ & 10.0 & $5.38 E+10$ & 23.6 & $6.77 E+10$ & 24.8 & .7 & $\cos 9(\mathrm{~N}, 3 \mathrm{~N}) \cos 7$ & $2.00 E+01$ & $6.00 \mathrm{E}+01$ \\
\hline 6 & $1.56 E+10$ & 10.0 & $2.48 E+10$ & 5.2 & $1.45 E+10$ & 7.0 & .0 & $\operatorname{co5} 9(\mathrm{~N}, 4 \mathrm{~N}) \operatorname{co5} 6$ & $3.20 E+01$ & $1.50 \mathrm{E}+02$ \\
\hline 7 & $1.29 E+13$ & 15.0 & $1.08 E+13$ & 16.3 & $1.22 E+13$ & 5.1 & .1 & $=559(\mathrm{~N}, \mathrm{G}) \mathrm{C060}$ & $2.30 \mathrm{E}-08$ & $31.00 \mathrm{E}-04$ \\
\hline 8 & $6.49 E+10$ & 15.0 & $7.21 E+10$ & -11.1 & $7.03 E+10$ & -8.2 & .2 & $N I 50(N, P) C 060$ & $5.00 \mathrm{E}+00$ & $1.20 \mathrm{E}+02$ \\
\hline 9 & $1.34 E+10$ & 10.0 & $1.15 E+10$ & 13.8 & $1.17 \mathrm{E}+10$ & 12.7 & .4 & $\mathrm{CU}(\mathrm{N}, *) 54 \mathrm{MN}$ & $8.80 E+01$ & $6.80 E+02$ \\
\hline 10 & $2.88 E+09$ & 25.0 & $9.09 E+08$ & 68.5 & $9.15 E+08$ & 68.3 & 7.1 & $\mathrm{CU}(\mathrm{N}, *) 59 \mathrm{FE}$ & $6.40 E+01$ & $6.80 E+02$ \\
\hline 11 & $6.90 E+09$ & 10.0 & $8.26 E+09$ & -19.8 & $8.23 E+09$ & -19.3 & .8 & $\mathrm{CU}: \mathrm{N}, *) 56 \mathrm{CO}$ & $6.40 \mathrm{E}+01$ & $6.40 E+02$ \\
\hline 12 & $3.00 E+10$ & 10.0 & $3.51 E+10$ & -17.0 & $3.48 E+10$ & -15.9 & .9 & $\mathrm{CU}: \mathrm{N}, * 157 \mathrm{CO}$ & $4.00 E+01$ & $6.00 \mathrm{E}+02$ \\
\hline 13 & $4.50 E+10$ & 10.0 & $4.82 E+10$ & -7.2 & $4.77 E+10$ & -6.0 & .2 & $\mathrm{CU}(\mathrm{N}, *) 58 \mathrm{CO}$ & $2.80 \mathrm{E}+01$ & $6.00 \mathrm{E}+02$ \\
\hline 14 & $2.56 \mathrm{E}+10$ & 25.0 & $9.94 E+09$ & 61.2 & $9.87 E+09$ & 61.5 & 5.8 & $\mathrm{CU}: \mathrm{N}, *) 60 \mathrm{CO}$ & $3.60 E+01$ & $6.40 \mathrm{E}+02$ \\
\hline 15 & $1.58 \mathrm{E}+12$ & 20.0 & $2.58 E+12$ & .1 & $1.59 E+12$ & -.1 & .0 & N393 (N, N') NB93M & $5.00 \mathrm{E}-01$ & $6.00 \mathrm{E}+00$ \\
\hline & & & .33 & & & & & & & \\
\hline & CHISQ & $=$ & 6.35 & & & & & & & \\
\hline
\end{tabular}

SUMMARY OF INTEGRATED GROUP ENERGY FLUXES AND FLUENCES

$\begin{array}{cccccr}\begin{array}{c}\text { ENERGY } \\ \mathrm{MeV}\end{array} & \begin{array}{c}\text { FLUX } \\ \mathrm{n} / \mathrm{Cm} 2-\mathrm{s}-\mathrm{mA}\end{array} & \begin{array}{c}\text { FLUX } \\ \mathrm{n} / \mathrm{Cm} 2-\mathrm{s}\end{array} & \begin{array}{r}\text { ELUENCE } \\ \mathrm{a \textrm {Cm } 2}\end{array} & \begin{array}{c}\text { STANDARD } \\ \text { DEVIATION }\end{array} & \\ & & & & & \\ <1.0 & 8.636 \mathrm{E}+12 & 2.653 \mathrm{E}+12 & 1.581 \mathrm{E}+19 & +1-8.29 & 29.95 \\ 0.1-1.0 & 1.223 \mathrm{E}+13 & 3.757 \mathrm{E}+12 & 2.381 \mathrm{E}+19 & +1-5.55 & 42.41 \\ 1.0-10.0 & 6.571 \mathrm{E}+12 & 2.019 \mathrm{E}+12 & 1.279 \mathrm{E}+19 & +1-9.18 & 22.78 \\ 10.0-20.0 & 2.376 \mathrm{E}+11 & 7.300 \mathrm{E}+10 & 4.625 \mathrm{E}+17 & +1-11.93 & .82 \\ 20.0-40.0 & 2.436 \mathrm{E}+11 & 7.485 \mathrm{E}+10 & 4.742 \mathrm{E}+17 & +1-12.21 & .84 \\ 40.0-100.0 & 2.935 \mathrm{E}+11 & 9.016 \mathrm{E}+10 & 5.722 \mathrm{E}+17 & +1-16.66 & 1.02 \\ 100.0-200.0 & 1.584 \mathrm{E}+11 & 4.866 \mathrm{E}+10 & 3.083 \mathrm{E}+17 & +1-21.95 & .55 \\ >200.0 & 4.252 \mathrm{E}+11 & 1.306 \mathrm{E}+11 & 8.277 \mathrm{E}+17 & +1-20.34 & 1.47 \\ \text { TOTAL } & 2.884 \mathrm{E}+13 & 8.860 \mathrm{E}+12 & 5.624 \mathrm{E}+19 & +1-6.63 & \end{array}$

THE PERCENTAGE OF NEUTRONS ABOVE $20 \mathrm{MeV}$ IS - 3.89 


\section{SUMMARY CALCULATIONS}

FOR 3-1 TO 3-5

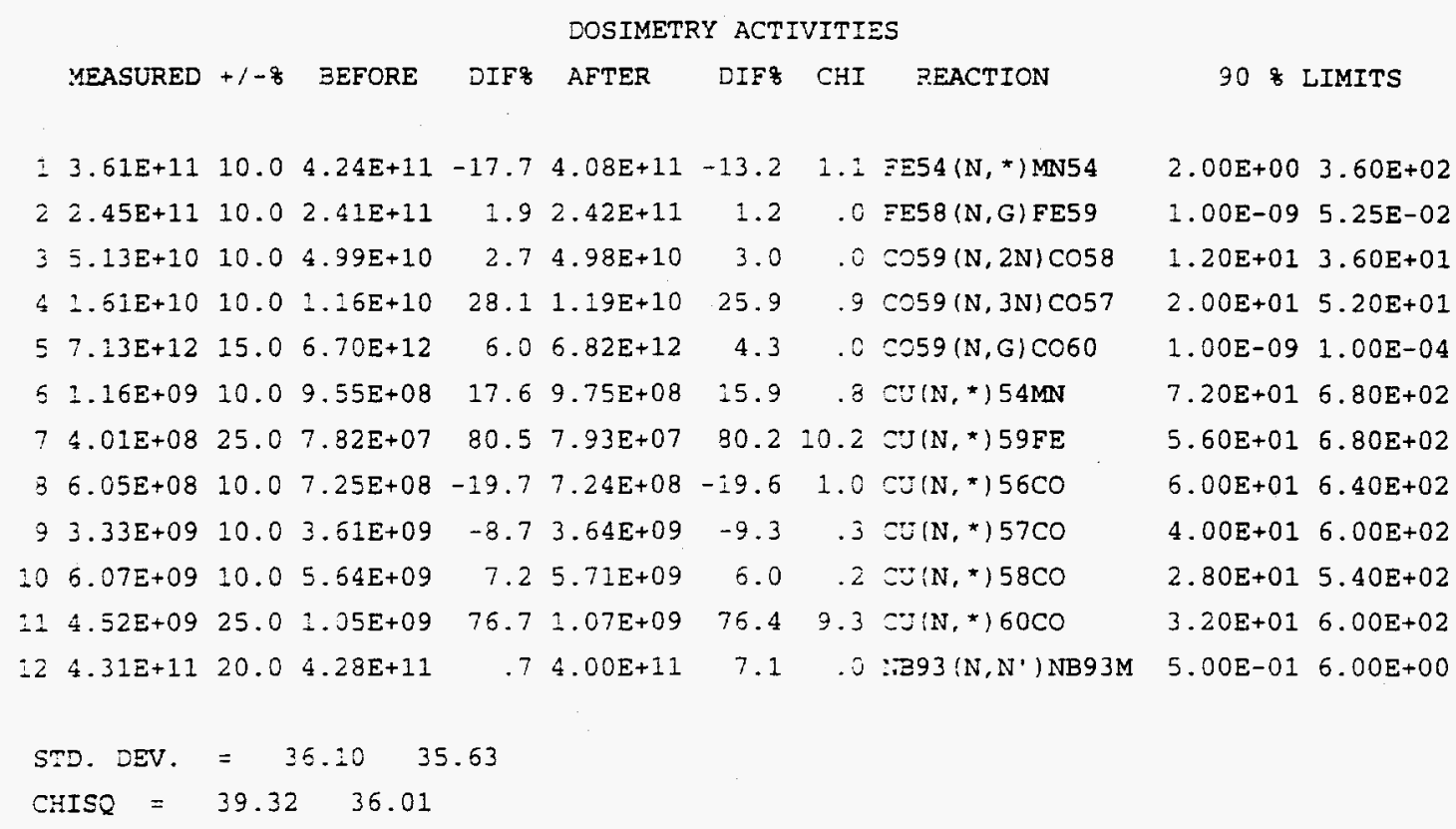

SUMMARY OF INTEGRATED GROUP ENERGY FLUXES AND FLUENCES

\begin{tabular}{|c|c|c|c|c|c|}
\hline $\begin{array}{c}\text { ENERGY } \\
\text { MeV }\end{array}$ & $\begin{array}{l}\text { FLUX } \\
\mathrm{n} / \mathrm{cm} 2-\mathrm{s}-\mathrm{mA}\end{array}$ & $\begin{array}{l}\text { FLUX } \\
\mathrm{n} / \mathrm{cm} 2-\mathrm{s}\end{array}$ & $\begin{array}{r}\text { ELUENCE } \\
\quad \mathrm{z} / \mathrm{cm} 2\end{array}$ & \multicolumn{2}{|c|}{$\begin{array}{l}\text { STANDARD } \\
\text { DEVIATION }\end{array}$} \\
\hline$<1.0$ & $2.809 E+12$ & $8.630 \mathrm{E}+11$ & $5.468 E+18$ & $+1-8.58$ & 36.34 \\
\hline $0.1-1.0$ & $3.088 \mathrm{E}+12$ & $9.488 \mathrm{E}+11$ & $6.52 E+18$ & $+1-5.88$ & 39.95 \\
\hline $1.0-10.0$ & $1.634 \mathrm{E}+12$ & $5.021 E+11$ & $3 .: 32 E+18$ & $+/-10.14$ & 21.14 \\
\hline $10.0-20.0$ & $6.158 \mathrm{E}+10$ & $1.892 \mathrm{E}+10$ & $1 .: 99 \mathrm{E}+17$ & $+1-12.98$ & .80 \\
\hline $20.0-40.0$ & $4.596 E+10$ & $1.412 \mathrm{E}+10$ & $8.947 E+16$ & $+/-12.54$ & .59 \\
\hline $40.0-100.0$ & $3.890 \mathrm{E}+10$ & $1.195 \mathrm{E}+10$ & $7 . \equiv: 3 E+16$ & $+1-16.43$ & .50 \\
\hline $00.0-200.0$ & $1.424 \mathrm{E}+10$ & $4.376 E+09$ & $2.773 E+16$ & $+1-22.09$ & .18 \\
\hline$>200.0$ & $3.355 E+10$ & $1.031 E+10$ & $6.531 E+16$ & $+1-22.42$ & .43 \\
\hline TOTAL & $7.730 \mathrm{E}+12$ & $2.375 E+12$ & $2.305 E+19$ & $+1-7.08$ & \\
\hline
\end{tabular}

THE PERCENTAGE OF NEUTRONS ABOVE $20 \mathrm{MeV}$ IS -1.72 


\section{SUMMARY CALCULATIONS \\ FOR 4-1 TO 4-5}

\begin{tabular}{|c|c|c|c|c|c|c|c|c|c|}
\hline & & & & & DOSIMETF & RY $A C^{\prime}$ & ITIE & & \\
\hline & MEASURED & $+1-8$ & BEFORE & DIF $\%$ & AFTER & $D I F \&$ & CHI & BEACTION & $90 \%$ LIMITS \\
\hline 1 & $3.15 E+11$ & 10.0 & $9.12 E+11$ & -11.9 & $9.01 \mathrm{E}+11$ & -10.5 & .5 & $\Xi \Xi 54(\mathrm{~N}, *) \mathrm{MN} 54$ & $2.00 E+00 \quad 5.00 E+02$ \\
\hline 2 & $3.20 \mathrm{E}+11$ & 10.0 & $2.91 \Xi+11$ & 8.9 & $3.08 E+11$ & 3.8 &. $\bar{z}$ & $\equiv \Xi 58(N, G) F E 59$ & $1.00 \mathrm{E}-092.00 \mathrm{E}-01$ \\
\hline 3 & 1. $\pm 0 E+11$ & 10.0 & $1.04 E+11$ & 5.7 & $1.04 E+11$ & 5.5 & $\therefore$ & $\cos 9(N, 2 N) \cos 8$ & $1.20 \mathrm{E}+01 \quad 3.60 \mathrm{E}+01$ \\
\hline 4 & $3.54 E+10$ & 10.0 & $2.93 E+10$ & 19.6 & $2.93 E+10$ & 19.6 & .4 & $\cos 9(N, 3 N) \operatorname{Cos} 7$ & $2.00 E+01 \quad 5.60 \mathrm{E}+01$ \\
\hline 5 & $9.50 E+12$ & 15.0 & $8.82 \Sigma+12$ & 7.1 & $9.47 \mathrm{E}+12$ & .3 & $\hat{\therefore}$ & $=059(N, G) \cos 0$ & $1.00 E-09 \quad 1.00 E-04$ \\
\hline 6 & $2.96 E+10$ & 15.0 & $3.12 \Xi+10$ & -5.3 & $3.07 E+10$ & -3.8 & .1 & $:: I 60(N, P) \cos 0$ & $5.00 \mathrm{E}+00 \quad 4.80 \mathrm{E}+01$ \\
\hline 7 & $3.912+09$ & 10.0 & $3.44 E+09$ & 12.0 & $3.47 \pm+09$ & 11.3 & .2 & $\sigma U(N, *) 54 M N$ & $8.80 E+01 \quad 7.20 E+02$ \\
\hline 8 & $1.06 \mathrm{E}+09$ & 25.0 & $2.71 \Xi+08$ & 74.4 & $2.71 \mathrm{E}+08$ & 74.4 & 8.5 & $\sigma(N, *) 59 F E$ & $6.00 \mathrm{E}+01 \quad 7.20 \mathrm{E}+02$ \\
\hline 9 & $2.03 \Xi+09$ & 10.0 & $2.37 E+09$ & -17.1 & $2.34 E+09$ & -15.4 & $\therefore$ & $\theta U(N, *) 56 C O$ & $6.40 \mathrm{E}+01 \quad 6.80 \mathrm{E}+02$ \\
\hline 10 & $9.755+09$ & 10.0 & $1.09 E+10$ & -11.4 & $1.07 \pm+10$ & -9.4 & .6 & $\approx(N, *) 57 C 0$ & $4.00 \mathrm{E}+01 \quad 6.40 \mathrm{E}+02$ \\
\hline 11 & $1.60 \mathrm{E}+10$ & 10.0 & $1.61 E+10$ & -.5 & $1.59 \mathrm{E}+10$ & .7 & .0 & $\mathrm{CU}(\mathrm{N}, *) 58 \mathrm{CO}$ & $2.80 \mathrm{E}+01 \quad 5.00 \mathrm{E}+02$ \\
\hline 2 & $1.04 \mathrm{E}+10$ & 25.0 & $3.16 E+09$ & 69.5 & $3.13 E+09$ & 59.8 & 7.6 & $\mathrm{CU}(\mathrm{N}, *) 60 \mathrm{CO}$ & $3.20 \mathrm{E}+01 \quad 6.40 \mathrm{E}+02$ \\
\hline 13 & $8.57 \Sigma+11$ & 20.0 & $8.55 E+11$ & .3 & $8.45 E+11$ & 1.5 & .0 & $: \operatorname{IB9} 3\left(N, N^{\prime}\right) N B 93 M$ & $5.00 \mathrm{E}-015.00 \mathrm{E}+00$ \\
\hline & 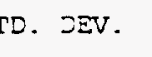 & $=$ & 31.15 & & & & & & \\
\hline & GISQ & $=$ & 28.99 & & & & & & \\
\hline
\end{tabular}

SUMMARY OF INTEGRATED GROUP ENERGY ZIUXES AND FLUENCES

\begin{tabular}{|c|c|c|c|c|c|}
\hline $\begin{array}{c}\text { ENERGY } \\
\text { MeV }\end{array}$ & $\begin{array}{c}=\mathrm{LUX} \\
\mathrm{n} / \mathrm{cm} 2-\mathrm{s}-\mathrm{mA}\end{array}$ & $\begin{array}{l}\text { ELUX } \\
\mathrm{n} / \mathrm{cm} 2-\mathrm{s}\end{array}$ & $\begin{array}{r}\text { FUENCE } \\
\mathrm{n} / \mathrm{cm} 2\end{array}$ & \multicolumn{2}{|c|}{$\begin{array}{l}\text { STANDARD } \\
\text { DEVIATION }\end{array}$} \\
\hline$<1.0$ & $4.537 E+12$ & $1.394 \mathrm{E}+12$ & $8.332 E+18$ & $+1-8.28$ & 30.08 \\
\hline $0.1-1.0$ & $6.524 E+12$ & $2.004 \mathrm{E}+12$ & $1.270 E+19$ & $+/-5.65$ & 43.25 \\
\hline $1.0-10.0$ & $3.530 \mathrm{E}+12$ & $1.084 \mathrm{E}+12$ & $6.371 \mathrm{E}+18$ & $+1-9.19$ & 23.40 \\
\hline $10.0-20.0$ & $1.022 \mathrm{E}+11$ & $3.141 E+10$ & 1. $990 \mathrm{E}+17$ & $+/-11.75$ & .68 \\
\hline $20.0-40.0$ & $1.118 \mathrm{E}+11$ & $3.434 E+10$ & $2 .: 76 \Xi+17$ & $+1-12.60$ & .74 \\
\hline $40.0-100.0$ & $9.480 \mathrm{E}+10$ & $2.912 E+10$ & I. $345 E+17$ & $+/-17.81$ & .63 \\
\hline $00.0-200.0$ & $3.787 E+10$ & $1.164 E+10$ & $7.372 \equiv+16$ & $+1-24.70$ & .25 \\
\hline$>200.0$ & $1.292 \mathrm{E}+11$ & $3.969 \mathrm{E}+10$ & $2.515 E+17$ & $+1-20.54$ & .86 \\
\hline TOTAL & $1.508 \mathrm{E}+13$ & $4.634 \mathrm{E}+12$ & $2.336 E+19$ & $+1-6.69$ & \\
\hline
\end{tabular}

THE PERCENTAGE OF NEUTRONS ABOVE $20 \mathrm{ueV}=\mathrm{s}-2.48$ 


\section{SUMMARY CALCULATIONS \\ FOR 5-1 TO 5-5}

\begin{tabular}{|c|c|c|c|c|c|c|c|c|}
\hline & & & & DOSIMET & RY ACT & IVITI: & & \\
\hline MEASURED & $+1-8$ & BEFORE & $\operatorname{SIF} 8$ & AFTER & DIF 8 & $\mathrm{CHI}$ & EEACTION & 90 LIMITS \\
\hline$I 5.95 \mathrm{E}+11$ & 10.0 & $7.57 E+11$ & -27.3 & $7.36 E+11$ & -23.8 & 2.8 & FE54(N, *) MN54 & $2.00 E+00 \quad 4.40 E+02$ \\
\hline $25.37 E+10$ & 10.0 & $2.25 \Xi+10$ & 58.2 & $2.31 E+10$ & 57.0 & 10.0 & $\cos 9(N, 3 N) \cos 7$ & $2.00 \mathrm{E}+01 \quad 5.20 \mathrm{E}+01$ \\
\hline $32.04 \mathrm{E}+13$ & 15.0 & $1.02 \Xi+13$ & 2.3 & $9.81 E+12$ & 5.7 & .0 & $\operatorname{Co5} 9(N, G) \operatorname{Co6} 0$ & $1.00 \mathrm{E}-08 \quad 1.00 \mathrm{E}-04$ \\
\hline $43.79 E+10$ & 15.0 & $3.47 E+10$ & 3.4 & $3.35 E+10$ & 12.5 & .3 & $N I 60(N, P) C 060$ & $5.00 E+00 \quad 2.80 E+01$ \\
\hline $52.85 E+09$ & 10.0 & $2.23 \Xi+09$ & 21.5 & $2.27 E+09$ & 20.5 & 1.3 & $\mathrm{CU}(\mathrm{N}, * 154 \mathrm{MN}$ & $3.00 \mathrm{E}+017.20 \mathrm{E}+02$ \\
\hline $66.64 \mathrm{E}+09$ & 10.0 & $7.73 \equiv+09$ & -16.5 & $7.61 E+09$ & -14.7 & .9 & $C v(N, * 157 C 0$ & $4.00 \mathrm{E}+016.40 \mathrm{E}+02$ \\
\hline $71.31 E+10$ & 25.0 & $2.25 \Xi+09$ & 82.8 & $2.23 E+09$ & 32.9 & 10.9 & $C U: 2 *^{* 160 C O}$ & $3.20 \mathrm{E}+016.40 \mathrm{E}+02$ \\
\hline $81.06 \mathrm{E}+12$ & 20.0 & $6.53 E+11$ & 38.7 & $5.17 E+11$ & $\div 2.0$ & .4 & NB93 $\{\mathrm{N}, \mathrm{N} \cdot \mid \mathrm{NB9} 3 \mathrm{M}$ & $5.00 \mathrm{E}-01 \quad 6.00 \mathrm{E}+00$ \\
\hline D. DEV. & $=$ & 43.58 & 3.52 & & & & & \\
\hline CHISQ & $=$ & 53.71 & 0.64 & & & & & \\
\hline
\end{tabular}

SUMMARY OF INTEGRATED GROUP ENERGY FLUXES AND ELUENCES

\begin{tabular}{|c|c|c|c|c|c|}
\hline $\begin{array}{c}\text { ENERGY } \\
\text { MeV }\end{array}$ & $\begin{array}{c}\text { FLUX } \\
\mathrm{n} / \mathrm{cm} 2-\mathrm{s}-\mathrm{mA}\end{array}$ & $\begin{array}{l}\text { ELUX } \\
n / \operatorname{cm} 2-5\end{array}$ & $\begin{array}{r}\text { SUENCE } \\
\quad=/ \operatorname{cm} 2\end{array}$ & \multicolumn{2}{|c|}{$\begin{array}{l}\text { STANDARD } \\
\text { DEVIATION }\end{array}$} \\
\hline$<1.0$ & $4.292 \mathrm{E}+12$ & 1. $318 E+12$ & $8.354 \Xi+18$ & $+/-11.01$ & 34.88 \\
\hline $0.1-1.0$ & $5.105 E+12$ & 1. $568 \mathrm{E}+12$ & $9.9375+18$ & $+1-6.20$ & 41.49 \\
\hline $1.0-10.0$ & $2.509 E+12$ & $7.709 E+11$ & $4.884 \bar{E}+18$ & $+1-10.01$ & 20.39 \\
\hline $10.0-20.0$ & $1.119 E+11$ & $3.437 E+10$ & $2.177 \equiv+17$ & $+/-14.66$ & .91 \\
\hline $20.0-40.0$ & $9.094 E+10$ & $2.794 E+10$ & $1.770 \Xi+17$ & $+/-18.08$ & .74 \\
\hline $40.0-100.0$ & $7.411 E+10$ & $2.2775+10$ & $1.443 \Xi+17$ & $+1-20.43$ & .50 \\
\hline $00.0-200.0$ & $3.022 E+10$ & $9.285 E+09$ & $5.883 \Xi+16$ & $+/-26.14$ & .25 \\
\hline$>200.0$ & $8.014 E+10$ & $2.462 \mathrm{E}+10$ & $1.550 \equiv+17$ & $+1-24.56$ & .05 \\
\hline TOTAL & $1.230 E+13$ & $3.780 \Xi+12$ & $2 \cdot 395 \equiv+19$ & $+1-8.07$ & \\
\hline
\end{tabular}

THE PERCENTAGE OF NEUTRONS ABOVE $20 \mathrm{MeV}=\$-2.24$

NOTE: The measured gamma-ray data from RAYGUN and SPECANL indicate that the identification of the peaks may be ambiguous. Thus, the results of this foil package should be used with caution. 


\section{SUMMARY CALCULATIONS}

FOR 6-1 TO 6-5

\begin{tabular}{|c|c|c|c|c|c|c|c|c|c|}
\hline & & & & & DOSIMETE & RY ACTI & VITI & & \\
\hline & MEASURED & $+1-3$ & BEFORE & $D I F$ \% & AFTER & DIF & $\mathrm{CHI}$ & REACTION & 90 LIMITS \\
\hline 1 & $7.26 E+11$ & 10.0 & $7.80 E+11$ & -3.9 & $7.50 E+11$ & -4.6 & .2 & FE54 (N, *) MN54 & $2.00 \mathrm{E}+00 \quad 4.20 \mathrm{E}+02$ \\
\hline 2 & $2.72 E+11$ & 10.0 & $2.70 E+11$ & .7 & $2.69 E+11$ & .9 & .0 & $F E 58(N, G) F E 59$ & $2.30 E-08 \quad 2.00 E-01$ \\
\hline 3 & $9.49 E+10$ & 10.0 & $1.01 E+11$ & -7.0 & $9.52 E+10$ & -.3 & .0 & $\cos 9(\mathrm{~N}, 2 \mathrm{~N}) \cos 8$ & $1.20 E+01 \quad 3.60 E+01$ \\
\hline 4 & $3.54 \mathrm{E}+10$ & 10.0 & $2.77 E+10$ & 22.0 & $2.59 E+10$ & 27.0 & .7 & $\cos 9(N, 3 N) \cos 7$ & $2.00 E+015.20 E+01$ \\
\hline 5 & $8.00 E+12$ & 15.0 & $7.80 E+12$ & 2.5 & $7.84 E+12$ & 2.1 & .0 & $\cos 9(\mathrm{~N}, \mathrm{G}) \cos 6$ & $2.30 \mathrm{E}-08 \quad 1.00 \mathrm{E}-04$ \\
\hline 6 & $2.54 E+10$ & 15.0 & $2.78 E+10$ & -9.8 & $2.62 \mathrm{E}+10$ & -3.2 & .1 & :II $60(\mathrm{~N}, \mathrm{P}) \mathrm{CO} 60$ & $5.00 E+00 \quad 4.00 E+01$ \\
\hline 7 & $2.93 E+09$ & 10.0 & $2.26 E+09$ & 22.8 & $2.49 E+09$ & 15.1 & 1.2 & $\mathrm{CU}(\mathrm{N}, *) 54 \mathrm{MN}$ & $7.60 \mathrm{E}+01 \quad 6.80 \mathrm{E}+02$ \\
\hline 8 & $8.53 E+08$ & 25.0 & $1.84 E+08$ & 78.5 & $1.98 E+08$ & 75.8 & 9.4 & $\sigma U(\mathrm{~N}, * / 59 \mathrm{FE}$ & $5.60 E+01 \quad 6.80 E+02$ \\
\hline 9 & $2.56 \mathrm{E}+09$ & 10.0 & $1.69 E+09$ & -8.1 & $1.78 E+09$ & -14.1 & .3 & $\mathrm{CU}(\mathrm{N}, *) 56 \mathrm{CO}$ & $6.40 E+01 \quad 6.40 E+02$ \\
\hline 10 & $7.72 \mathrm{E}+09$ & 10.0 & $8.50 E+09$ & -10.1 & $8.54 E+09$ & -10.6 & .4 & $\mathrm{CU}(\mathrm{N}, *) 57 \mathrm{CO}$ & $4.00 E+01 \quad 6.00 E+02$ \\
\hline 1 & $\pm .32 \mathrm{E}+10$ & 10.0 & $1.33 E+10$ & -.9 & $1.31 \mathrm{E}+10$ & .9 & .0 & $\mathrm{CU}(\mathrm{N}, *) 58 \mathrm{CO}$ & $2.80 \mathrm{E}+015.40 \mathrm{E}+02$ \\
\hline 2 & $8.97 E+09$ & 25.0 & $2.49 E+09$ & 72.3 & $2.50 E+09$ & 72.2 & 8.2 & $\mathrm{CU}(\mathrm{N}, *) 60 \mathrm{CO}$ & $3.20 \mathrm{E}+01 \quad 6.00 \mathrm{E}+02$ \\
\hline 3 & 7. $40 E+11$ & 20.0 & $7.33 E+11$ & .9 & $7.08 \mathrm{E}+11$ & 4.3 & .0 & NB9 $3\left(N, N^{\prime}\right)$ NB9 3M & $5.00 \mathrm{E}-015.00 \mathrm{E}+00$ \\
\hline & 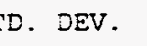 & $=$ & 32.03 & 2.19 & & & & & \\
\hline & ZHISQ & $=$ & 31.62 & & & & & & \\
\hline
\end{tabular}

SUMMARY OF INTEGRATED GROUP ENERGY FLUXES AND FLUENCES

\begin{tabular}{|c|c|c|c|c|c|}
\hline $\begin{array}{c}\text { ENERGY } \\
\text { MeV }\end{array}$ & $\begin{array}{c}\text { FLUX } \\
\mathrm{n} / \mathrm{cm} 2-\mathrm{s}-\mathrm{mA}\end{array}$ & $\begin{array}{l}\text { FLUX } \\
\mathrm{n} / \mathrm{cm} 2-\mathrm{s}\end{array}$ & $\begin{array}{r}\text { ELUENCE } \\
n / \mathrm{cm} 2\end{array}$ & \multicolumn{2}{|c|}{$\begin{array}{l}\text { STANDARD } \\
\text { DEVIATION }\end{array}$} \\
\hline$<1.0$ & $4.197 E+12$ & $1.289 \mathrm{E}+12$ & $8 .: 70 E+18$ & $+/-7.91$ & 31.99 \\
\hline $0.1-1.0$ & $5.591 E+12$ & 1. $718 \mathrm{E}+12$ & $1.088 E+19$ & $+/-5.47$ & 42.61 \\
\hline $1.0-10.0$ & $2.921 E+12$ & $8.974 \mathrm{E}+11$ & $5.686 \mathrm{E}+18$ & $+1-9.00$ & 22.25 \\
\hline $10.0-20.0$ & $9.752 \mathrm{E}+10$ & $2.996 \mathrm{E}+10$ & $1.898 E+17$ & $+/-11.42$ & .74 \\
\hline $20.0-40.0$ & $9.934 E+10$ & $3.052 \mathrm{E}+10$ & $1.234 E+17$ & $+/-11.68$ & .76 \\
\hline $40.0-100.0$ & $8.390 E+10$ & $2.578 E+10$ & $1.633 \mathrm{E}+17$ & $+/-15.88$ & .64 \\
\hline $00.0-200.0$ & $3.318 \mathrm{E}+10$ & $1.019 \mathrm{E}+10$ & $6.459 E+16$ & $+/-22.33$ & .25 \\
\hline$>200.0$ & $8.996 E+10$ & $2.754 \mathrm{E}+10$ & $2.751 E+17$ & $+/-21.87$ & .59 \\
\hline TOTAL & $1.312 \mathrm{E}+13$ & $4.031 E+12$ & $2.554 E+19$ & $+1-6.52$ & \\
\hline
\end{tabular}

THE PERCENTAGE OF NEUTRONS ABOVE $20 \mathrm{MeV}$ IS - 2.33 


\section{APPENDIX IV}

STAY'SL Output Calculations 


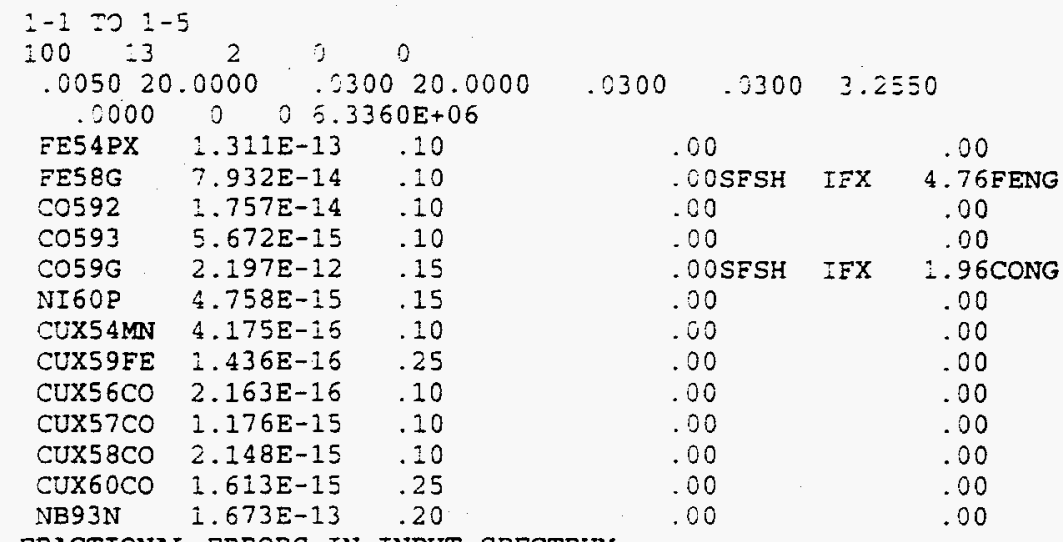

0 FRACTIONAL ERRORS IN INPUT SPECTRUM

$161.000 E+00$

$1.000 \mathrm{E}-11 \quad 1.000 \mathrm{E}-07 \quad 1.000 \mathrm{E}-05 \quad 1.000 \mathrm{E}-03 \quad 1.000 \mathrm{E}-02 \quad 1.020 \mathrm{E}-01 \quad 4.000 \mathrm{E}-01 \quad 1.000 \mathrm{E}+00$ $4.000 E+001.000 E+01 \quad 2.000 E+01 \quad 4.400 E+01 \quad 1.000 E+02 \quad 4.200 E+02 \quad 8.000 E+02 \quad 0.000 E+00$ 5.000E-01 4.000E-01 2.800E-01 2.000E-01 1.500E-01 1.:20E-01 8.000E-02 8.000E-02 $2.500 \mathrm{E}-013.000 \mathrm{E}-01 \quad 3.500 \mathrm{E}-01 \quad 4.000 \mathrm{E}-01 \quad 5.000 \mathrm{E}-01 \quad 6.200 \mathrm{E}-01 \quad 0.000 \mathrm{E}+00 \quad 0.000 \mathrm{E}+00$

1 INPUT SPECTRUM

$1001.000 E+00$

$\begin{array}{lllllllll}4.678 \mathrm{E}+17 & 3.151 \mathrm{E}+17 & 2.489 \mathrm{E}+17 & 2.471 \mathrm{E}+17 & 2.446 \mathrm{E}+17 & 2.274 \mathrm{E}+17 & 1.673 \mathrm{E}+17 & 1.060 \mathrm{E}+17\end{array}$

$5.290 \mathrm{E}+16 \quad 2.640 \mathrm{E}+16 \quad 1.284 \mathrm{E}+16 \quad 5.377 \mathrm{E}+15 \quad 2.735 \mathrm{E}+15 \quad 1.527 \mathrm{E}+15 \quad 7.962 \mathrm{E}+14 \quad 3.566 \mathrm{E}+14$

$\begin{array}{llllllll}1.748 E+14 & 1.026 E+14 & 7.664 E+13 & 5.731 E+13 & 4.285 E+13 & 3.384 E+13 & 2.025 E+13 & 1.131 E+13\end{array}$

$9.898 \mathrm{E}+12 \quad 4.824 \mathrm{E}+12 \quad 2.507 \mathrm{E}+12 \quad 1.109 \mathrm{E}+12 \quad 5.560 \mathrm{E}+11 \quad 3.555 \mathrm{E}+11 \quad 1.700 \mathrm{E}+11 \quad 8.141 \mathrm{E}+10$

$3.594 \mathrm{E}+10 \quad 2.498 \mathrm{E}+10 \quad 2.757 \mathrm{E}+10 \quad 1.186 \mathrm{E}+10 \quad 9.622 \mathrm{E}+09 \quad 8.2 \mathrm{2} 2 \mathrm{E}+09 \quad 6.925 \mathrm{E}+09 \quad 5.856 \mathrm{E}+09$

$5.399 \mathrm{E}+09 \quad 5.099 \mathrm{E}+09 \quad 4.831 \mathrm{E}+09 \quad 4.589 \mathrm{E}+09 \quad 4.394 \mathrm{E}+09 \quad 4.228 \mathrm{E}+09 \quad 3.734 \mathrm{E}+09 \quad 3.163 \mathrm{E}+09$

$2.717 E+092.368 \mathrm{E}+092.052 \mathrm{E}+09 \quad 1.789 \mathrm{E}+09 \quad 1.575 \mathrm{E}+09 \quad 1.578 \mathrm{E}+09 \quad 1.201 \mathrm{E}+091.039 \mathrm{E}+09$

$9.009 E+08 \quad 7.643 E+08 \quad 6.569 E+08 \quad 5.532 E+08 \quad 4.840 E+08 \quad 4.223 E+08 \quad 3.569 E+08 \quad 3.165 E+08$

$2.788 \mathrm{E}+08 \quad 2.473 \mathrm{E}+08 \quad 2.218 \mathrm{E}+08 \quad 1.905 \mathrm{E}+08 \quad 1.742 \mathrm{E}+08 \quad 1 . \Sigma=1 \mathrm{E}+08 \quad 1.477 \mathrm{E}+08 \quad 1.390 \mathrm{E}+08$

$1.341 E+08 \quad 1.279 E+08 \quad 1.206 E+08 \quad 1.182 E+08 \quad 1.235 E+08 \quad 1.256 E+08 \quad 1.006 E+08 \quad 9.800 E+07$

$9.952 \mathrm{E}+07 \quad 9.951 \mathrm{E}+07 \quad 9.973 \mathrm{E}+07 \quad 1.018 \mathrm{E}+08 \quad 1.006 \mathrm{E}+08 \quad 1.2 \mathrm{0E}+08 \quad 9.693 \mathrm{E}+07 \quad 9.337 \mathrm{E}+07$

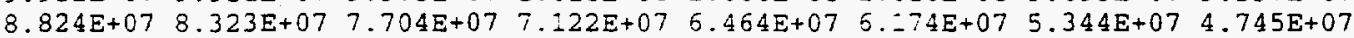

$4.228 E+07 \quad 3.782 E+07 \quad 3.343 E+07 \quad 2.919 E+07$

\begin{tabular}{|c|c|c|c|c|c|}
\hline \multirow{3}{*}{$\begin{array}{c}100 \quad \text { NFOIL }= \\
\text { REACTIONS }\end{array}$} & $\$ 3$ & ACTYYTY NORM= & \multicolumn{3}{|c|}{$3.255 \mathrm{E}+00$} \\
\hline & COVER & $\therefore$ CTIVITIES & & F-SHIF & DING \\
\hline & .00 & $\therefore .267 E-13$ & & & \\
\hline FE58 (N, G)FE59 & .00 & $=.582 E-13$ & IFX & EENG & 4.76 \\
\hline $\cos 9(N, 2 N) \cos 8$ & .00 & $\Xi .719 \mathrm{E}-14$ & & & .00 \\
\hline $\cos 9(\mathrm{~N}, 3 \mathrm{~N}) \cos 7$ & .00 & $=.846 \mathrm{E}-14$ & & & .00 \\
\hline $\cos 9(\mathrm{~N}, \mathrm{G}) \cos 0$ & .00 & $-.251 \mathrm{E}-12$ & IFX & CONG & 1.9 \\
\hline NI $60(\mathrm{~N}, \mathrm{P}) \mathrm{CO} 60$ & .00 & $\therefore 549 \mathrm{E}-14$ & & & .0 \\
\hline$C U(N, \bullet) 54 M N$ & .00 & $=.359 \mathrm{E}-15$ & & & .0 \\
\hline $\mathrm{CU}(\mathrm{N}, *) 59 \mathrm{FE}$ & .00 & $\therefore .674 \mathrm{E}-16$ & & & .0 \\
\hline $\mathrm{CU}(\mathrm{N}, *) 56 \mathrm{CO}$ & .00 & $-.341 E-16$ & & & .0 \\
\hline $\operatorname{CU}(\mathrm{N}, *) 57 \mathrm{CO}$ & .00 & $\Xi .828 \mathrm{E}-15$ & & & .0 \\
\hline $\mathrm{CU}(\mathrm{N}, *) 58 \mathrm{CO}$ & .00 & $\Xi .992 \mathrm{E}-15$ & & & .0 \\
\hline $\mathrm{CU}(\mathrm{N}, *) 60 \mathrm{CO}$ & .00 & $\equiv .250 \mathrm{E}-15$ & & & .0 \\
\hline $\operatorname{NB9} 3\left(\mathrm{~N}, \mathrm{~N}^{\prime}\right) \mathrm{NB} 93 \mathrm{M}$ & .00 & $\equiv .446 E-13$ & & & 0 \\
\hline
\end{tabular}

\section{COVARIANCE PARAMETERS}

ELUXES: WIDTH $=2.0000 E+01 \quad$ FCOV $=3.0000 E-02$

ACTIVITIES $=5.0000 \mathrm{E}-03$ CROSS SECTIONS $=3.6200 \mathrm{E}-02$

DETERM $=3.787 * 10 *-20.00$ ERROR $=-3.196 \mathrm{E}-14$

INPUT NORMALIZATION DATA

AK1 $=\quad .0000$ VAK $=\quad .00090 \mathrm{NORM}=0$ RENORM $=\quad .5568 \mathrm{cHI} 2=23.7512$

DOSIMETRY ACTIVITIES

MEASURED $+/-\frac{8}{3}$ BEFORE DIF\% AFTER DIF\& CHI EZACTION $\quad 90 \%$ LIMTTS

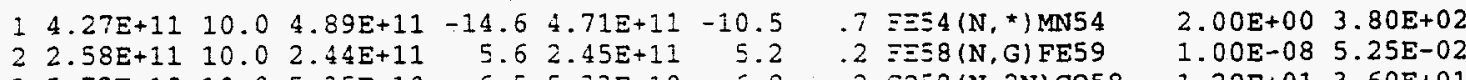

$35.72 \mathrm{E}+10 \quad 10.0 \quad 5.35 \mathrm{E}+10 \quad 6.5 \quad 5.33 \mathrm{E}+10 \quad 6.8 \quad .2 \cos (\mathrm{N}, 2 \mathrm{~N}) \operatorname{Co5} 8 \quad 1.20 \mathrm{E}+01 \quad 3.60 \mathrm{E}+01$ 
$41.85 E+10 \quad 10.0 \quad 1.42 E+10 \quad 23.3 \quad 1.45 E+10.21 .7$

$57.15 E+12 \quad 15.0 \quad 8.12 E+i 2-13.5 \quad 8.21 E+12-14.8$

$\begin{array}{lllllll}5 & 1.55 E+10 & 15.0 & 1.77 E+10 & -14.5 & 1.67 E+10 & -7.5\end{array}$

$71.35 E+09 \quad 10.0 \quad 1.08 E+09 \quad 20.6 \quad 1.14 E+09 \quad: 5.4$

$34.67=+0825.01 .08 E+09$

$97.04 E+08 \quad 10.0 \quad 8.03 E+08-14$

$103.83 E+09 \quad 10.0 \quad 4.16 E+09$

$116.99 E+0910.06 .61 E+09$

$125.25 E+0925.0 \quad 1.22 E+09$

$135.45 E+1120.0 \quad 5.41 E+11$

$$
\begin{aligned}
& \text { STD. SEV. = 34.56 } 34.05 \\
& \text { ZHISQ }=37.28 \quad 34.22
\end{aligned}
$$

DOSIMETRY DATA INPUT CORRELATION MATRIX

$$
\begin{aligned}
& 11000
\end{aligned}
$$

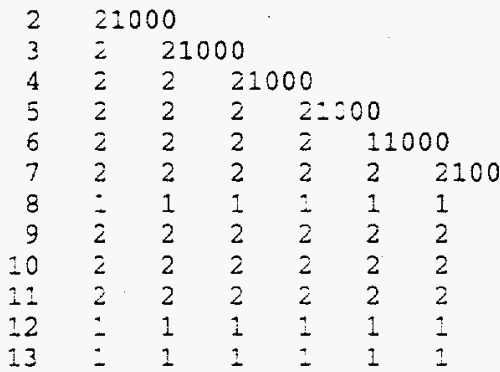

$.6 \operatorname{co5} 9(\mathrm{~N}, 3 \mathrm{~N}) \cos 7$

$.20059(N, G) C 060$

.3 NII $60(\mathrm{~N}, \mathrm{P}) \mathrm{CO} 60$

$1.0 \mathrm{CU}(\mathrm{N}, *) 54 \mathrm{MN}$

$0.2 \operatorname{CU}(\mathrm{N}, * 159 \mathrm{FE}$

$.6 \mathrm{CU}(\mathrm{N}, *) 56 \mathrm{CO}$

$.4 \operatorname{Cu}(\mathrm{N}, *) 57 \mathrm{CO}$

$.1 \mathrm{CU}\left(\mathrm{N},{ }^{*}\right) 58 \mathrm{CO}$

$9.3 \mathrm{CU}(\mathrm{N}, *) 50 \mathrm{CO}$

.0 NB93 (N, N') NB93M
$2.00 \mathrm{E}+01 \quad 5.20 \mathrm{E}+01$

1. $00 \mathrm{E}-08$ 1.00E-04

$5.00 \mathrm{E}+002.80 \mathrm{E}+01$

$5.80 E+01 \quad 6.80 E+02$

$5.60 E+01 \quad 6.80 E+02$

$5.00 E+01 \quad 6.40 E+02$

$4.00 \mathrm{E}+01 \quad 5.60 \mathrm{E}+02$

$2.80 E+01 \quad 5.20 E+02$

$3.20 E+016.00 E+02$

$5.00 E-01 \quad 5.00 E+00$

REIATIYE COV. MATRIX CE ACTIVITIES

3 COFRELATION MATRIX

125.221000

$225.19 \quad 2441000$

335.698131121000

$452.46 \quad 590 \quad 446431000$

$536.08 \quad 168715 \quad 03261000$

$6 \begin{array}{lllll}30.88 & 779 & 246 & 777 & 475 \quad 1651000\end{array}$

$\begin{array}{llllllll}7 & 44.69 & 322 & 18 & 13 & 158 & 12 & 981000\end{array}$

$\begin{array}{lllllllll}3 & 41.60 & 394 & 21 & 203 & 230 & 13 & 143 & 8061000\end{array}$

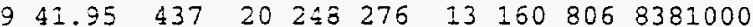

$\begin{array}{lllllllllll}10 & 36.66 & 643 & 32 & 524 & 505 & 19 & 344 & 682 & 754 & 8141000\end{array}$

$\begin{array}{lllllllllllll}11 & 35.31 & 738 & 43 & 681 & 610 & 25 & 471 & 555 & 641 & 706 & 8881000\end{array}$

$\begin{array}{lllllllllllll}12 & 35.52 & 671 & 36 & 569 & 530 & 22 & 384 & 660 & 733 & 790 & 905 & 8951000\end{array}$

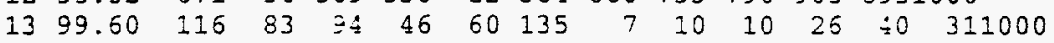

CONTRIBUTION DUE TO INPUT FLUX COV. MATRIX

CORRELATION MATRIX

123.351000

224.052601000

$3 \quad 33.7 i \quad 9181131000$

$437.83 \quad 873 \quad 549401000$

$\begin{array}{llllll}5 & 26.66 & 231 & 999 & 89 & 391000\end{array}$

$\begin{array}{lllllll}6 & 28.21 & 907 & 268 & 892 & 713 & 2331000\end{array}$

$\begin{array}{llllllll}7 & 39.97 & 379 & 12 & 152 & 239 & 9 & 1121000\end{array}$

$\begin{array}{lllllllll}8 & 37.54 & 459 & 14 & 239 & 347 & 11 & 165 & 9911000\end{array}$

$938.99 \quad 498 \quad 23 \quad 27 \equiv 406 \quad 20 \quad 180 \quad 9649901000$

$\begin{array}{lllllllllll}10 & 34.89 & 719 & 25 & 575729 & 17 & 387 & 795 & 869 & 9131000\end{array}$

$\begin{array}{llllllllllll}11 & 33.75 & 822 & 36 & 7 \div 5 & 877 & 26 & 530 & 643 & 734 & 788 & 9681000\end{array}$

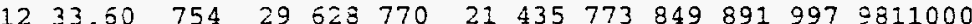

$\begin{array}{llllllllllllll}13 & 15.65 & 726 & 498 & 57 & 364 & 462 & 865 & 32 & 52 & 53 & 149 & 235 & 1791000\end{array}$

CONTRIBUTION DUE TO INPUT X-SEC. COV. MATRIX

$$
\text { S CORRELATION MATRIX }
$$

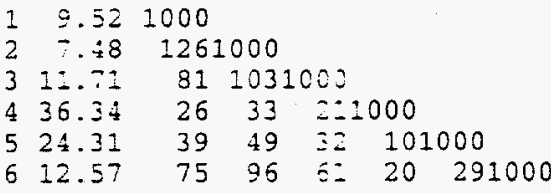




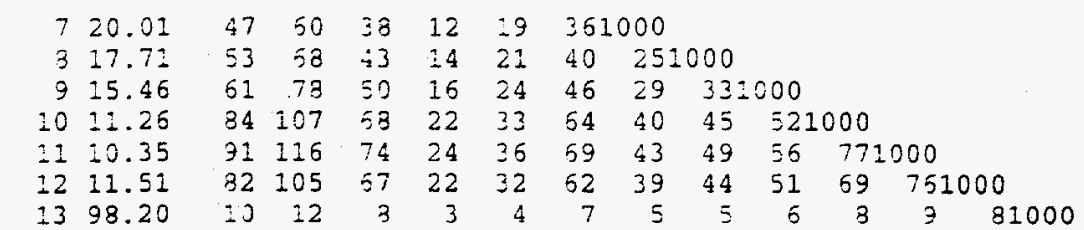

$\begin{array}{ccccc}\text { DIFFERENTIAL FLUXES } & \text { ZNPUT NORMALIZED } & \text { BY } & .9668 \\ \text { G ENERGY } & \text { NEW } & \text { OLD } & \text { RATIO } & \text { STD DEV }\end{array}$

i $\quad .000 \mathrm{E}-10 \quad 4.943 \mathrm{E}+17 \quad 4.523 \mathrm{E}+17$

$\therefore .000 E-09$

RATIO

9668

$1.093 \quad 30.88$

1.087

$2.601 E+17 \quad 2.406 E+17$

2. $300 \mathrm{E}-08$

5. $000 \mathrm{E}-08$

1. $150 \mathrm{E}-07$

2.571

i. 081

1. 075

2. $531 E+17 \quad 2.365 E+17$

2. $550 \mathrm{E}-07$

2. $233 \mathrm{E}+17$

2. $102 E+17$

1.070

1. 062

1. 053

1. $703 \mathrm{E}+17$ 1.617E+17

$5.500 \mathrm{E}-07$

?. $275 E-06$

$1.069 \mathrm{E}+17$

1. $025 \mathrm{E}+17$

1. 043

2. $515 E+16$

5. $114 \mathrm{E}+16$

1.034

2. $552 E+16$

6. $300 \mathrm{E}-06$

1. $350 \mathrm{E}-05$

3. $000 \mathrm{E}-05$

5. $722 \mathrm{E}+15$

1. $241 \mathrm{E}+16$

2. $541 E+15$

5. $682 \mathrm{E}+15$

.025

1.015

1.007

2. $544 \mathrm{E}+15$

1. $000 \mathrm{E}-04$

2. $800 E-04$

5. $750 E-04$

1. $540 E+15$

1. $554 \mathrm{E}+15$

$7.698 \mathrm{E}+14$

3. $370 E+14$

3. $448 \mathrm{E}+14$

$.542 E+14$

1. $690 \mathrm{E}+14$

1. $275 E-03$
2. $000 E-03$

3. $400 E-03$

7. $133 E+13$

9. $919 E+13$

5. $500 E-03$

5. $316 E+13$

7. $410 \mathrm{E}+13$

3. $965 \mathrm{E}+13$

$5.541 \mathrm{E}+13$

9. $200 \mathrm{E}-03$

I. $200 \mathrm{E}-02$

3. $586 E+13$

4. $143 E+13$

3. $755 E+13$

1. $875 \mathrm{E}+13 \quad 1.958 \mathrm{E}+13$

5.250 $\mathrm{E}-02$

1. $053 E+13$

1. $093 \mathrm{E}+13$

I. $000 \mathrm{E}-01$

9. $235 E+12$

9. $569 E+12$

5. $000 E-01$

2. $348 E+12$

4. $664 \mathrm{E}+12$

$\therefore .000 E+00$

1. $025 \mathrm{E}+12$

2. $424 \mathrm{E}+12$

2. $000 \mathrm{E}+00$

1. $072 \mathrm{E}+12$

$=.014 E+11$

$5.375 E+11$

3. $000 \mathrm{E}+00$

2. $704 \mathrm{E}+11$

2. $963 \mathrm{E}+11$

4.000E+00

$7.104 E+10$

$.644 \mathrm{E}+11$

999

.991

.984

972

967

.963

959

.957

.955

958

.963

965

.967

969

.956

.933

.912

.903

.903

$6.000 E+00$

3. $143 E+10$

$71 \mathrm{E}+10$

7. $000 E+00$

2. $194 \mathrm{E}+10$

2. $415 \mathrm{E}+10$

.905

908

8. $000 E+00$

1. $552 \mathrm{E}+10$

1. $699 \mathrm{E}+10$

1. $000 E+01$

8. $640 E+09$

1. $147 \mathrm{E}+10$

.914
.921

.929

i. $100 \mathrm{E}+01$

7. $444 \mathrm{E}+09$

$9.303 \mathrm{E}+09$

$7.939 \mathrm{E}+09$

6. $695 \mathrm{E}+09$

1. $200 E+01$

5. $342 \mathrm{E}+09$

5. $662 \mathrm{E}+09$

$400 \mathrm{E}+01$

5. $420 \mathrm{E}+09$

5. $220 \mathrm{E}+09$

$5.050 \mathrm{E}+09$

$4.930 \mathrm{E}+09$

$4.611 \mathrm{E}+09$

$4.671 \mathrm{E}+09$

1. $600 \mathrm{E}+01$

4. $419 E+09$

4. $437 \mathrm{E}+09$

1. $800 \mathrm{E}+01$

4. $266 \mathrm{E}+09$

$4.248 \mathrm{E}+09$

1. $900 \mathrm{E}+01$

4. $133 E+09$

$4.088 \mathrm{E}+09$

$3.671 E+09 \quad 3.610 E+09$

2. $000 E+01$

3. $124 \mathrm{E}+09$

3. $058 \mathrm{E}+09$

2. $800 \mathrm{E}+01$

2. $691 \mathrm{E}+09$

2. $627 \mathrm{E}+09$

3. $200 \mathrm{E}+01$

2. $349 E+09$

2. $289 E+09$

$3.600 \mathrm{E}+01$

2.035E+09

$1.984 \mathrm{E}+09$

1. $730 \mathrm{E}+09$

4. $400 \mathrm{E}+01$

1. $552 \mathrm{E}+09$

1. $521 \mathrm{E}+09$

1. $353 E+09$

1. $332 \mathrm{E}+09$

5. $200 \mathrm{E}+01$

1. $172 \mathrm{E}+09$

1. $161 \mathrm{E}+09$

$5.600 E+01$

1.007E+09

1. $005 \mathrm{E}+09$

8. $710 \mathrm{E}+08$

6. $000 E+01$
$6.400 E+01$

7. $275 E+08$

7. $389 \mathrm{E}+08$

$6.800 E+01$

5. $193 E+08$

6. $351 \mathrm{E}+08$

7. $200 E+01$

5. $165 \mathrm{E}+08$

5. $348 \mathrm{E}+08$

$7.600 E+01$

4. $476 E+08$

4. $679 \mathrm{E}+08$

.938

.947

.957

.967

.977

.987

.996

1.004

1.011

1.017

i. 022

1.025

1.026

1. 026

27.78

25.38

23.58

21.83

19.83

17.80

16.06

14.63

13.51

12.66

12.04

11.57

11. 37

11.15

10.84

10.55

10.23

9.92

9.55

9.17

8. 84

7.80

6.58

5.97

5.42

5.14

6.61

9.57

12.23

13.55

13.64

13.60

13.47

13.27

13.02

12.75

12.51

12.25

12.01

11.80

11.62

11.49

11. 41

11.39

11.42

11.64

12.02

12.43

12.88

13.39

$.024 \quad 13.94$

1. 021

14.62

15.40

1.009

16.22

17.08

994

17.95

18.83

19.70

20.56

21.37

$3.992 \mathrm{E}+08$

22.14

22.84

38100

(2)

NEW

OLD

RATIO

INT FLUX >+-8

$46.08 \quad 670 \quad 8.598 E+12$

$43.59 \quad .637 \quad 8.597 \mathrm{E}+12$

$41.96 \quad .605 \quad 8.594 \mathrm{E}+12$

$41.15 \quad .573 \quad 8.591 \mathrm{E}+12$

$40.22 \quad .543 \quad 8.584 \mathrm{E}+12$

$38.49 \quad .515 \quad 8.567 \mathrm{E}+12$

$35.24 \quad .491 \quad 8.536 \mathrm{E}+12$

$34.06 \quad .471 \quad 8.486 \mathrm{E}+12$

$32.00 \quad .457 \quad 8.408 \mathrm{E}+12$

$30.10 \quad .449 \quad 8.328 \mathrm{E}+12$

$28.35 \quad .446 \quad 8.236 \mathrm{E}+12$

$26.77 \quad .450 \quad 8.146 \mathrm{E}+12$

$25.25 \quad .458 \quad 8.051 E+12$

$24.17 \quad .470 \quad 7.948 \mathrm{E}+12$

$22.99 \quad .485 \quad 7.900 \mathrm{E}+12$

$21.59 \quad .502 \quad 7.764 \mathrm{E}+12$ 


$\begin{array}{rllll}54 & 3.800 E+01 & 2.357 E+08 & 3.060 E+08 & .334 \\ 55 & 9.200 E+01 & 2.502 E+08 & 2.695 E+08 & .928 \\ 56 & 9.500 E+01 & 2.211 E+08 & 2.391 E+08 & .925 \\ 57 & 1.500 E+02 & 1.979 E+08 & 2.144 E+08 & .923 \\ 58 & 1.200 E+02 & 1.699 E+08 & 1.342 E+08 & .322 \\ 59 & 1.200 E+02 & 1.557 E+08 & 1.684 E+08 & .324 \\ 70 & 1.300 E+02 & 1.445 E+08 & 1.558 E+08 & .928 \\ 71 & 1.400 E+02 & 1.333 E+08 & 1.428 E+08 & .934 \\ 72 & 1.500 E+02 & 1.265 E+08 & 1.344 E+08 & .941 \\ 73 & 1.500 E+02 & 1.232 E+08 & 1.296 E+08 & .950 \\ 74 & 1.7 j 0 E+02 & 1.188 E+08 & 1.237 E+08 & .951 \\ 75 & 1.300 E+02 & 1.335 E+08 & 1.166 E+08 & .973 \\ 76 & 1.300 E+02 & 1.127 E+08 & 1.143 E+08 & .987 \\ 77 & 2.000 E+02 & 1.098 E+08 & 1.097 E+08 & 1.001 \\ 78 & 2.200 E+02 & 1.037 E+08 & 1.021 E+08 & 1.016 \\ 79 & 2.400 E+02 & 1.004 E+08 & 9.726 E+07 & 1.032 \\ 80 & 2.600 E+02 & 9.928 E+07 & 9.475 E+07 & 1.048 \\ 81 & 2.800 E+02 & 1.024 E+08 & 9.622 E+07 & 1.064 \\ 82 & 3.000 E+02 & 1.039 E+08 & 9.621 E+07 & 1.080 \\ 83 & 3.200 E+02 & 1.056 E+08 & 9.642 E+07 & 1.095 \\ 84 & 3.500 E+02 & 1.093 E+08 & 9.842 E+07 & 1.120 \\ 85 & 3.500 E+02 & 1.093 E+08 & 9.726 E+07 & 1.124 \\ 86 & 3.500 E+02 & 1.110 E+08 & 9.765 E+07 & 1.137 \\ 87 & 4.500 E+02 & 1.072 E+08 & 9.371 E+07 & 1.144 \\ 88 & 4.200 E+02 & 1.034 E+08 & 9.027 E+07 & 1.146 \\ 89 & 4.400 E+02 & 9.764 E+07 & 8.531 E+07 & 1.145 \\ 90 & 4.600 E+02 & 9.185 E+07 & 8.047 E+07 & 1.141 \\ 91 & 4.800 E+02 & 8.466 E+07 & 7.448 E+07 & 1.137 \\ 92 & 5.000 E+02 & 7.782 E+07 & 6.886 E+07 & 1.130 \\ 93 & 5.200 E+02 & 7.014 E+07 & 6.249 E+07 & 1.122 \\ 94 & 5.400 E+02 & 6.647 E+07 & 5.969 E+07 & 1.114 \\ 95 & 5.600 E+02 & 5.680 E+07 & 5.167 E+07 & 1.099 \\ 96 & 6.000 E+02 & 4.956 E+07 & 4.588 E+07 & 1.080 \\ 97 & 6.400 E+02 & 4.338 E+07 & 4.088 E+07 & 1.061 \\ 98 & 6.300 E+02 & 3.812 E+07 & 3.656 E+07 & 1.043 \\ 99 & 7.230 E+02 & 3.312 E+07 & 3.232 E+07 & 1.025 \\ 100 & 7.500 E+02 & 2.844 E+07 & 2.822 E+07 & 1.008\end{array}$

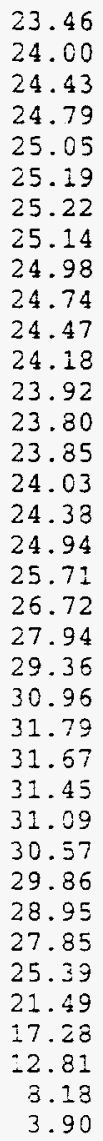

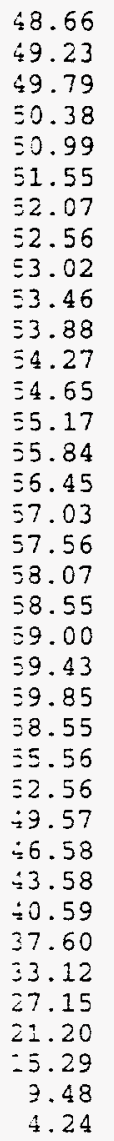

.482
.487
.491
.492
.491
.489
.484
.478
.471
.463
.454
.446
.438
.431
.427
.426
.428
.433
.443
.456
.474
.494
.517
.543
.570
.598
.627
.656
.685
.713
.741
.767
.791
.815
.838
.863
.920
.

$6.204 \mathrm{E}+10$

14.82

$5.090 \mathrm{E}+10$

15.19

$24.43 \quad 49.79 \quad .491 \quad 5.990 \mathrm{E}+10$

15.51

$5.901 E+10$

15.80

$91 \quad 5.704 \mathrm{E}+10 \quad 16.46$

$\begin{array}{lll}.489 & 5.534 E+10 \quad 17.05\end{array}$

$5.378 E+10 \quad 17.62$
$.478-5.234 E+10 \quad 18.15$

$478 \quad 5.234 \mathrm{E}+10 \quad 18.15$

$5.100 E+10 \quad 18.65$

$463 \quad 4.974 E+10 \quad 19.12$

$446 \quad 4.732 E+10 \quad 19.97$

$438 \quad 4.618 \mathrm{E}+10 \quad 20.34$

$431 \quad 4.506 \mathrm{E}+10 \quad 20.68$

$427 \quad 4.286 E+10 \quad 21.31$

$\begin{array}{lll}4.078 E+10 & 21.89\end{array}$

$428 \quad 3.878 \mathrm{E}+10 \quad 22.41$

$\begin{array}{lll}.433 & 3.679 \mathrm{E}+10 \quad 22.88\end{array}$

$443 \quad 3.474 \mathrm{E}+10 \quad 23.30$
.456

$\begin{array}{lll}.456 & 3.267 \mathrm{E}+10 \quad 23.64\end{array}$

$474 \quad 3.055 \mathrm{E}+10 \quad 23.88$

$.494 \quad 2.837 \mathrm{E}+10 \quad 24.00$

$.517 \quad 2.618 \mathrm{E}+10 \quad 23.94$

$\begin{array}{lll}.543 & 2.396 \mathrm{E}+10 & 23.65\end{array}$

$\begin{array}{lll}570 & 2.182 \mathrm{E}+10 & 23.17\end{array}$

$\begin{array}{lll}598 & 1.975 E+10 & 22.55\end{array}$

$\begin{array}{lll}627 & 1.780 \mathrm{E}+10 & 21.80\end{array}$

$.656 \quad 1.596 \mathrm{E}+10 \quad 20.92$

$\begin{array}{lll}685 & 1.427 \mathrm{E}+10 & 19.92\end{array}$

$\begin{array}{lll}.713 & 1.271 \mathrm{E}+10 & 18.81\end{array}$

$\begin{array}{lll}741 & 1.131 \mathrm{E}+10 & 17.63\end{array}$

$\begin{array}{lll}.767 & 9.977 \mathrm{E}+09 & 16.33\end{array}$

$7917.705 \mathrm{E}+09 \quad 13.74$

$815 \quad 5.723 \mathrm{E}+09 \quad 11.13$

$\begin{array}{lll}.838 & 3.988 \mathrm{E}+09 \quad 8.54\end{array}$

$2.463 E+09 \quad 6.05$

INTEGRALE OF SPECTRA

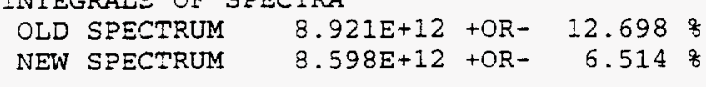

\begin{tabular}{|c|c|c|c|c|c|}
\hline ENERGY & ELUX & SDEV & ENERGY & FLUX & SDEV \\
\hline 1.000E-10 & $4.943 E+17$ & 30.88 & $3.600 E+01$ & $2.035 E+09$ & 13.39 \\
\hline I. $000 \mathrm{E}-09$ & $3.310 E+17$ & 27.78 & $4.000 E+01$ & $1.771 E+09$ & 13.94 \\
\hline I. $000 \mathrm{E}-08$ & $2.601 E+17$ & 25.38 & $4.400 \mathrm{E}+01$ & $1.552 \mathrm{E}+09$ & 14.62 \\
\hline $2.300 E-08$ & $2.571 \mathrm{E}+17$ & 23.58 & $4.800 E+01$ & $1.353 \mathrm{E}+09$ & 15.40 \\
\hline $5.000 E-08$ & $2.531 E+17$ & 21.83 & $5.200 E+0 i$ & $1.172 \mathrm{E}+09$ & 16.22 \\
\hline $1.250 \mathrm{E}-07$ & $2.233 E+17$ & 19.83 & $5.600 E+0:$ & $1.007 \mathrm{E}+09$ & 17.08 \\
\hline $2.550 \mathrm{E}-07$ & $1.703 E+17$ & 17.80 & $6.000 E+01$ & $8.654 E+08$ & 17.95 \\
\hline $5.500 \mathrm{E}-07$ & $1.069 \mathrm{E}+17$ & 16.06 & $6.400 E+0 i$ & $7.275 E+08$ & 18.83 \\
\hline $1.275 \mathrm{E}-06$ & $5.287 E+16$ & 14.63 & $6.800 E+0:$ & $6.193 E+08$ & 19.70 \\
\hline $2.800 \mathrm{E}-06$ & $2.615 E+16$ & 13.51 & $7.200 \mathrm{E}+0 \pm$ & $5.165 E+08$ & 20.56 \\
\hline $6.300 \mathrm{E}-06$ & $1.261 E+16$ & 12.66 & $7.600 E+03$ & $4.476 E+08$ & 21.37 \\
\hline I. 3500E-05 & $5.722 E+15$ & 12.04 & $8.000 E+0 I$ & $3.784 E+08$ & 22.14 \\
\hline $3.000 E-05$ & $2.641 E+15$ & 11.57 & $8.400 E+01$ & $3.244 E+08$ & 22.84 \\
\hline $6.900 \mathrm{E}-05$ & $1.540 E+15$ & 11.37 & $8.800 E \div 01$ & $2.857 E+08$ & 23.46 \\
\hline 1. $200 \mathrm{E}-04$ & $7.573 E+14$ & 11.16 & $9.200 E+0:$ & $2.502 E+08$ & 24.00 \\
\hline $2.800 E-04$ & $3.370 \mathrm{E}+14$ & 10.84 & $9.600 E+01$ & $2.211 E+08$ & 24.43 \\
\hline $5.750 \mathrm{E}-04$ & 1. $642 \mathrm{E}+14$ & 10.55 & $1.000 E+02$ & $1.979 E+08$ & 24.79 \\
\hline $1.275 \mathrm{E}-03$ & $9.590 \mathrm{E}+13$ & 10.23 & $1.100 E+02$ & $1.699 E+08$ & 25.05 \\
\hline $2.000 E-03$ & $7.133 E+13$ & 9.92 & $1.200 E+02$ & $1.557 E+08$ & 25.19 \\
\hline $3.400 \mathrm{E}-03$ & $5.316 E+13$ & 9.55 & $1.300 E+02$ & $1.445 E+08$ & 25.22 \\
\hline $5.500 \mathrm{E}-03$ & $3.965 E+13$ & 9.17 & $1.400 E+02$ & $1.333 E+08$ & 25.14 \\
\hline $9.200 \mathrm{E}-03$ & $3.586 E+13$ & 8.84 & $1.500 \mathrm{E}+02$ & $1.265 E+08$ & 24.98 \\
\hline $1.200 \mathrm{E}-02$ & $1.875 E+13$ & 7.80 & $1.600 \mathrm{E}+02$ & $1.232 \mathrm{E}+08$ & 24.74 \\
\hline $5.250 \mathrm{E}-02$ & $1.053 E+13$ & 6.58 & $1.700 E+02$ & $1.188 E+08$ & 24.47 \\
\hline $1.200 \mathrm{E}-01$ & $9.235 E+12$ & 5.97 & $1.800 \mathrm{E}+02$ & $1.135 \mathrm{E}+08$ & 24.18 \\
\hline 2. $000 \mathrm{E}-01$ & $4.512 E+12$ & 5.42 & $1.900 \mathrm{E}+02$ & $1.127 \mathrm{E}+08$ & 23.92 \\
\hline 5. $000 \mathrm{E}-01$ & $2.348 E+12$ & 5.14 & $2.000 E+02$ & $1.098 E+08$ & 23.80 \\
\hline 1. $000 \mathrm{E}+00$ & 1. $025 E+12$ & 6.61 & $2.200 E+02$ & $1.037 E+08$ & 23.85 \\
\hline $2.000 \mathrm{E}+00$ & $5.014 E+11$ & 9.57 & $2.400 E+02$ & $1.004 \mathrm{E}+08$ & 24.03 \\
\hline $3.000 E+00$ & $2.704 \mathrm{E}+11$ & 12.23 & $2.600 E+02$ & $9.928 E+07$ & 24.38 \\
\hline
\end{tabular}




\begin{tabular}{|c|c|c|c|c|c|}
\hline $4.000 E+00$ & i. $483 \mathrm{E}+11$ & 23.55 & $2.800 E+02$ & $1.024 E+08$ & 24.94 \\
\hline$\Xi .000 E+00$ & T. $154 \mathrm{E}+10$ & 13.54 & $\therefore .000 E+02$ & $1.039 E+08$ & 25.71 \\
\hline $5.000 E+00$ & $3.143 E+10$ & .3 .60 & 3. $200 E+02$ & $1.056 \mathrm{E}+08$ & 26.72 \\
\hline $7.000 E+00$ & $2.194 \mathrm{E}+10$ & $: 3.47$ & $3.400 E+02$ & $1.093 E+08$ & 27.94 \\
\hline $3.000 E+00$ & $\vdots .552 \mathrm{E}+10$ & 12.27 & $3.500 E+02$ & $1.093 E+08$ & 29.36 \\
\hline $9.000 E+00$ & $=.056 \mathrm{E}+10$ & 13.02 & $3.800 E+02$ & $1.110 E+08$ & 30.96 \\
\hline$\therefore .000 E+01$ & $3.640 \mathrm{E}+09$ & $\$ 2.76$ & $4.000 E+02$ & $1.072 E+08$ & 31.79 \\
\hline$\therefore 100 E+01$ & $7.444 E+09$ & 12.51 & $4.200 E+02$ & $1.034 E+08$ & 31.67 \\
\hline $1.200 E+01$ & $5.342 E+09$ & 12.25 & $4.400 E+02$ & $9.764 E+07$ & \\
\hline$=.300 E+01$ & $5.420 E+09$ & $=2.01$ & $4.600 E+02$ & $9.185 \mathrm{E}+07$ & 31.09 \\
\hline$\therefore .400 E+01$ & $5.050 E+09$ & 11.80 & $4.800 E+02$ & $8.466 E+07$ & 30.57 \\
\hline$\therefore .500 E+01$ & $4.319 E+09$ & 11.62 & $5.000 E+02$ & $7.782 E+07$ & 29.86 \\
\hline i. $600 \mathrm{E}+01$ & $4.611 E+09$ & 11.49 & $5.200 E+02$ & $7.014 E+07$ & 28.95 \\
\hline $1.700 E+01$ & 4. $419 E+09$ & 11.41 & $5.400 E+02$ & $6.647 E+07$ & 27.85 \\
\hline $\pm .800 \mathrm{E}+01$ & $4.266 \bar{E}+09$ & 11.39 & $5.600 E+02$ & $5.680 E+07$ & 25.39 \\
\hline $1.900 E+01$ & $4.133 E+09$ & 11.42 & $5.000 E+02$ & $4.956 \mathrm{E}+07$ & 21.49 \\
\hline $2.000 E+01$ & $3.671 E+09$ & -1.64 & $5.400 E+02$ & $4.338 E+07$ & 17.28 \\
\hline $2.400 E+01$ & 3. $124 E+09$ & 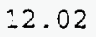 & $5.800 E+02$ & $3.812 \mathrm{E}+07$ & 12.81 \\
\hline $2.800 E+01$ & $2.691 \mathrm{E}+09$ & 12.43 & $7.200 E+02$ & $3.312 \mathrm{E}+07$ & 8.18 \\
\hline $3.200 E+01$ & $2.349 E+09$ & $: 2.88$ & $7.600 \mathrm{E}+02$ & $2.844 \mathrm{E}+07$ & \\
\hline
\end{tabular}

SUMMARY OF BROAD-GROUP FLUXES, FUUENCES, AND UNCERTAINTIES

\begin{tabular}{|c|c|c|c|c|}
\hline ENERGY & ELUX & EIUENCE & & SDEV \\
\hline OTAL & $8.598 E+12$ & $1.674 \mathrm{E}+19$ & $+1-$ & 5.51 \\
\hline $\begin{array}{l}1.000 E-10 \\
1.150 E-07 \\
3.350 E-05 \\
\therefore .000 E-01 \\
-.000 E+00 \\
. .000 E+01 \\
2.000 E+01 \\
4.000 E+01 \\
-.000 E+02 \\
2.000 E+02\end{array}$ & $\begin{array}{l}3.020 E+10 \\
4.219 E+11 \\
2.384 E+12 \\
3.451 E+12 \\
2.096 E+12 \\
5.514 E+10 \\
5.548 E+10 \\
4.596 E+10 \\
1.396 E+10 \\
4.107 E+10\end{array}$ & $\begin{array}{l}5.878 \mathrm{E}+16 \\
8.213 \mathrm{E}+17 \\
4.640 \mathrm{E}+18 \\
6.718 \mathrm{E}+18 \\
4.079 \mathrm{E}+18 \\
1.073 \mathrm{E}+17 \\
1.080 \mathrm{E}+17 \\
8.947 \mathrm{E}+16 \\
2.7: 7 \mathrm{E}+16 \\
7.994 \mathrm{E}+16\end{array}$ & $\begin{array}{l}+1- \\
-1- \\
+1- \\
-1- \\
+1- \\
-1- \\
-1- \\
+1- \\
-1- \\
+1-\end{array}$ & $\begin{array}{r}23.21 \\
-4.63 \\
3.22 \\
3.46 \\
8.90 \\
-1.21 \\
-1.12 \\
-2.12 \\
25.94 \\
23.59 \\
22.22\end{array}$ \\
\hline
\end{tabular}

SUMMARY OF INTEGRATED GROUP ENERGY FLUXES AND ILUENCES

\begin{tabular}{|c|c|c|c|c|c|}
\hline $\begin{array}{l}\text { ENERGY } \\
\text { MeV }\end{array}$ & $\begin{array}{c}\text { ELUX } \\
\mathrm{n} / \mathrm{cm} 2-\mathrm{s}-\mathrm{mA}\end{array}$ & $\begin{array}{c}\text { FIUX } \\
\mathrm{a} / \mathrm{cm} 2-\mathrm{s}\end{array}$ & $\begin{aligned} \text { ELUENCE } \\
\text { n/Cn2 }\end{aligned}$ & $\begin{array}{l}\text { STANDARD } \\
\text { DEVIATION }\end{array}$ & 8 \\
\hline $\begin{array}{c}<1.0 \\
0.1-1.0 \\
1.0-10.0 \\
10.0-20.0 \\
20.0-40.0 \\
40.0-100.0 \\
100.0-200.0 \\
>200.0 \\
\text { TOTAL }\end{array}$ & $\begin{array}{l}2.836 \mathrm{E}+12 \\
3.451 \mathrm{E}+12 \\
2.096 \mathrm{E}+12 \\
5.514 \mathrm{E}+10 \\
5.548 \mathrm{E}+10 \\
4.596 \mathrm{E}+10 \\
1.396 \mathrm{E}+10 \\
4.107 \mathrm{E}+10 \\
8.598 \mathrm{E}+12\end{array}$ & $\begin{array}{l}3.712 E+11 \\
1.060 E+12 \\
5.438 E+11 \\
1.694 E+10 \\
1.704 E+10 \\
1.412 E+10 \\
4.289 E+09 \\
1.262 E+10 \\
2.641 E+12\end{array}$ & $\begin{array}{l}5.520 \Xi+18 \\
6.718 E+18 \\
4.079 E+18 \\
1.073 E+17 \\
1.080 E+17 \\
8.947 E+16 \\
2.717 E+16 \\
7.994 E+16 \\
1.674 E+19\end{array}$ & $\begin{array}{l}-8.22 \\
-i-5.46 \\
-1-3.90 \\
-i-11.21 \\
-1-12.12 \\
-1-15.94 \\
-i-23.59 \\
-i-22.22 \\
-1-5.51\end{array}$ & $\begin{array}{r}32.98 \\
40.14 \\
24.37 \\
.64 \\
.65 \\
.53 \\
.16 \\
.48\end{array}$ \\
\hline
\end{tabular}

THE PERCENTAGE OF NEUTRONS ABOVE $20 \mathrm{MeV}$ IS - 1.82

RELATIVE COVARIANCES $(10 \times 10)$

$\begin{array}{rrrrrrrrrr}1000 & 843 & -21 & -208 & -208 & 7 & 108 & 63 & 7 & 12 \\ 1000 & 405 & 80 & 13 & 4 & 73 & 62 & 21 & 19 & \\ 1000 & 879 & 784 & 156 & -151 & -68 & 65 & 46 & & \\ 1000 & 941 & 313 & -189 & -147 & 72 & 70 & & \\ 1000 & 501 & -165 & -209 & 75 & 96 & & & \\ 1000 & 531 & -65 & -136 & 97 & & & & \\ 1000 & 608 & -221 & -217 & & & & & \\ 1000 & 298 & -554 & & & & & & & \\ 1000 & -161 & & & & & & & & \\ 1000 & & & & & & & & \end{array}$




\begin{tabular}{|c|c|c|c|c|c|c|c|}
\hline \multicolumn{8}{|c|}{$.0050 \quad 20.0000 \quad .0300^{0} 20.0000$} \\
\hline .0000 & 0 & 5 . 5. & $60 E+06$ & & & & \\
\hline EE54AX & $3.963 \mathrm{E}$ & $\varepsilon-13$ & .25 & & 00 & & .00 \\
\hline FE5 4PX & $5.173 E$ & $E-13$ & .10 & & 00 & & .00 \\
\hline EE58G & $1.531 \mathrm{E}$ & E-13 & .20 & & OOSESH & IFX & $4.76 \mathrm{FENG}$ \\
\hline $\cos 92$ & $7.447 \mathrm{E}$ & $E-14$ & .10 & & 00 & & .00 \\
\hline $\cos 93$ & $2.766 \mathrm{E}$ & $E-14$ & .20 & & 00 & & .00 \\
\hline $\cos 94$ & $4.783 \mathrm{E}$ & $E-15$ & .10 & & 00 & & .00 \\
\hline $\cos 9 G$ & $3.954 \mathrm{E}$ & $e-12$ & .25 & & OOSFSH & IFX & $\therefore . \exists 6 \mathrm{CONG}$ \\
\hline NI60P & $1.995 \mathrm{E}$ & $E-14$ & .15 & & 00 & & .00 \\
\hline $\operatorname{CUX} 54 \mathrm{MN}$ & $4.102 E$ & $2-15$ & .10 & & 00 & & .30 \\
\hline CUX59EE & $8.857 \mathrm{E}$ & $8-15$ & .25 & & 00 & & .00 \\
\hline $\operatorname{cux} 56 \mathrm{Co}$ & $2.119 \mathrm{E}$ & $\varepsilon-15$ & .10 & & 00 & & .00 \\
\hline cux57co & $\vartheta .223 \mathrm{E}$ & $2-15$ & .10 & & 00 & & .30 \\
\hline CUX58CO & $1.382 \mathrm{E}$ & $E-14$ & .10 & & 00 & & .00 \\
\hline CUX60CO & $7.870 \mathrm{E}$ & -15 & .25 & & 00 & & .00 \\
\hline NB93N & $4.867 \mathrm{E}$ & $8-13$ & .20 & & 00 & & .00 \\
\hline
\end{tabular}

2 FRACTIONAL ERRORS IN INPUT SPECTRUM

16. $1.000 \mathrm{E}+00$

$1.000 \mathrm{E}-11 \quad 1.000 \mathrm{E}-07 \quad 2.000 \mathrm{E}-05 \quad 1.000 \mathrm{E}-03 \quad 1.000 \mathrm{E}-02 \quad 2.300 \mathrm{E}-01 \quad 4.000 \mathrm{E}-01 \quad 1.000 \mathrm{E}+00$

$4.000 \mathrm{E}+001.000 \mathrm{E}+01 \quad 2.000 \mathrm{E}+01 \quad 4.400 \mathrm{E}+01 \quad 1.000 \mathrm{E}+02 \quad 4.000 \mathrm{E}+02 \quad 8.000 \mathrm{E}+02 \quad 0.000 \mathrm{E}+00$

5.000E-01 4.000E-01 2.800E-01 2.000E-01 1.500E-01 1. .00E-01 8.000E-02 8.000E-02

$2.500 E-013.000 E-01 \quad 3.500 E-01 \quad 4.000 E-01 \quad 5.000 E-01 \quad 5.300 E-01 \quad 0.000 E+00 \quad 0.000 E+00$

: INPUT SPECTRUM

$1001.000 E+00$

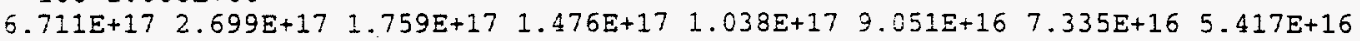
$4.063 E+16 \quad 2.354 E+16 \quad 1.650 E+16 \quad 8.976 E+15 \quad 4.037 E+15 \quad 2.587 E+15 \quad 1.291 E+15 \quad 6.190 E+14$ $3.096 \mathrm{E}+14 \quad 3.571 \mathrm{E}+14 \quad 2.803 \mathrm{E}+14 \quad 2.203 \mathrm{E}+14 \quad 1.728 \mathrm{E}+14 \quad \mathrm{i} .534 \mathrm{E}+14 \quad 6.687 \mathrm{E}+13 \quad 4.054 \mathrm{E}+13$ $2.922 E+13 \quad 1.724 E+13 \quad 8.080 E+12 \quad 3.411 E+12 \quad 1.502 E+12 \quad 7.5 \pm 2 E+11 \quad 3.658 E+11 \quad 2.122 E+11$

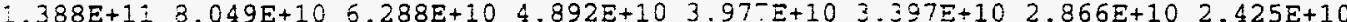
$2.238 \mathrm{E}+10 \quad 2.117 \mathrm{E}+10 \quad 2.012 \mathrm{E}+10 \quad 1.921 \mathrm{E}+10 \quad 1.853 \mathrm{E}+10 \quad 1.302 \mathrm{E}+10 \quad 1.607 \mathrm{E}+10 \quad 1.376 \mathrm{E}+10$ $1.202 E+10 \quad 1.072 E+10 \quad 9.557 E+09 \quad 8.634 E+09 \quad 7.895 E+09 \quad 7.237 E+09 \quad 6.626 E+09 \quad 6.065 E+09$ $5.593 \mathrm{E}+09 \quad 5.066 \mathrm{E}+09 \quad 4.665 \mathrm{E}+09 \quad 4.220 \mathrm{E}+09 \quad 3.971 \mathrm{E}+09 \quad 3.645 \mathrm{E}+09 \quad 3.387 \mathrm{E}+09 \quad 3.221 \mathrm{E}+09$ $2.849 \mathrm{E}+092.863 \mathrm{E}+09 \quad 2.496 \mathrm{E}+09 \quad 2.197 \mathrm{E}+09 \quad 1.946 \mathrm{E}+09 \quad 1.704 \mathrm{E}+09 \quad 1.591 \mathrm{E}+09 \quad 1.491 \mathrm{E}+09$ $\therefore .427 \mathrm{E}+09 \quad 1.367 \mathrm{E}+09 \quad 1.323 \mathrm{E}+09 \quad 1.292 \mathrm{E}+09 \quad 1.250 \mathrm{E}+09 \quad 1.33 \mathrm{E}+09 \quad 1.152 \mathrm{E}+09 \quad 1.123 \mathrm{E}+09$

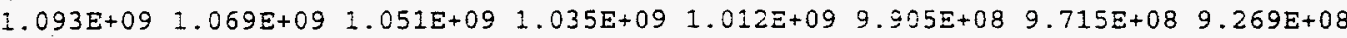
$3.947 E+08 \quad 8.445 E+08 \quad 8.004 E+08 \quad 7.490 E+08 \quad 6.980 E+08 \quad 6.540 E+08 \quad 5.891 E+08 \quad 5.205 E+08$ $4.601 E+08 \quad 4.005 E+08 \quad 3.524 E+08 \quad 3.113 E+08$

\begin{tabular}{|c|c|c|c|c|c|}
\hline \multirow{3}{*}{$\begin{aligned} \text { NGROUP } & =100 \text { NFOIL }= \\
& = \pm 54(\mathrm{NEACTIONS} \\
& \approx) \mathrm{CR} 51 .\end{aligned}$} & 15 & ACTIVITY NORM= & \multicolumn{3}{|c|}{$3.255 E+00$} \\
\hline & COVER & ACTIVITIES & & F-SHI & DING \\
\hline & .00 & $\pm .290 \mathrm{E}-12$ & & & .00 \\
\hline FE54(N, *) MN5 4 & .00 & $2.009 \Xi-12$ & & & 8 \\
\hline$\equiv \equiv 58(N, G)$ FE5 9 & .00 & $4.983 E-13$ & IFX & FENG & 4.7 \\
\hline $\cos 9(\mathrm{~N}, 2 \mathrm{~N}) \operatorname{Co5} 8$ & .00 & $2 . \div 24 E-13$ & & & .0 \\
\hline $\cos 9(\mathrm{~N}, 3 \mathrm{~N}) \operatorname{Co5} 7$ & .00 & $\Xi .003 E-14$ & & & $\cdot$ \\
\hline $\cos 9(\mathrm{~N}, 4 \mathrm{~N}) \cos 6$ & .00 & $\therefore .557 E-14$ & & & .6 \\
\hline $\operatorname{Co5} 9(N, G) \operatorname{co6} 0$ & .00 & $=.287 E-11$ & IFX & CONG & 1.9 \\
\hline NI $60(N, P) C 060$ & .00 & $\Xi .494 E-14$ & & &. \\
\hline $\mathrm{CU}(\mathrm{N}, *) 54 \mathrm{MN}$ & .00 & $=.335 E-14$ & & & .0 \\
\hline $\mathrm{CU}(\mathrm{N}, *) 59 \mathrm{FE}$ & .00 & $2.883 E-15$ & & & .0 \\
\hline $\mathrm{CU}(\mathrm{N}, *) 56 \mathrm{CO}$ & .00 & $\Xi .897 E-15$ & & & .0 \\
\hline $\mathrm{CU}(\mathrm{N}, \star) 57 \mathrm{CO}$ & .00 & $\Xi .002 E-14$ & & & .0 \\
\hline$C U(N, *) 58 \mathrm{CO}$ & .00 & $\div 498 \mathrm{E}-14$ & & & .0 \\
\hline $\mathrm{CU}(\mathrm{N}, *) 60 \mathrm{CO}$ & .00 & $2.552 \mathrm{E}-14$ & & & .00 \\
\hline J.। NB93M & .00 & $\therefore .534 \mathrm{E}-12$ & & & 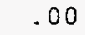 \\
\hline
\end{tabular}

\section{COVARIANCE PARAMETERS}

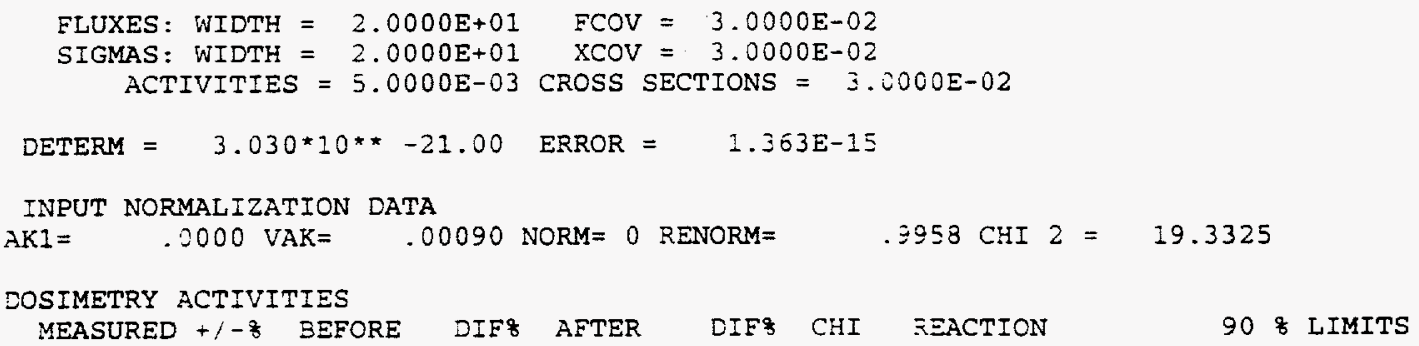




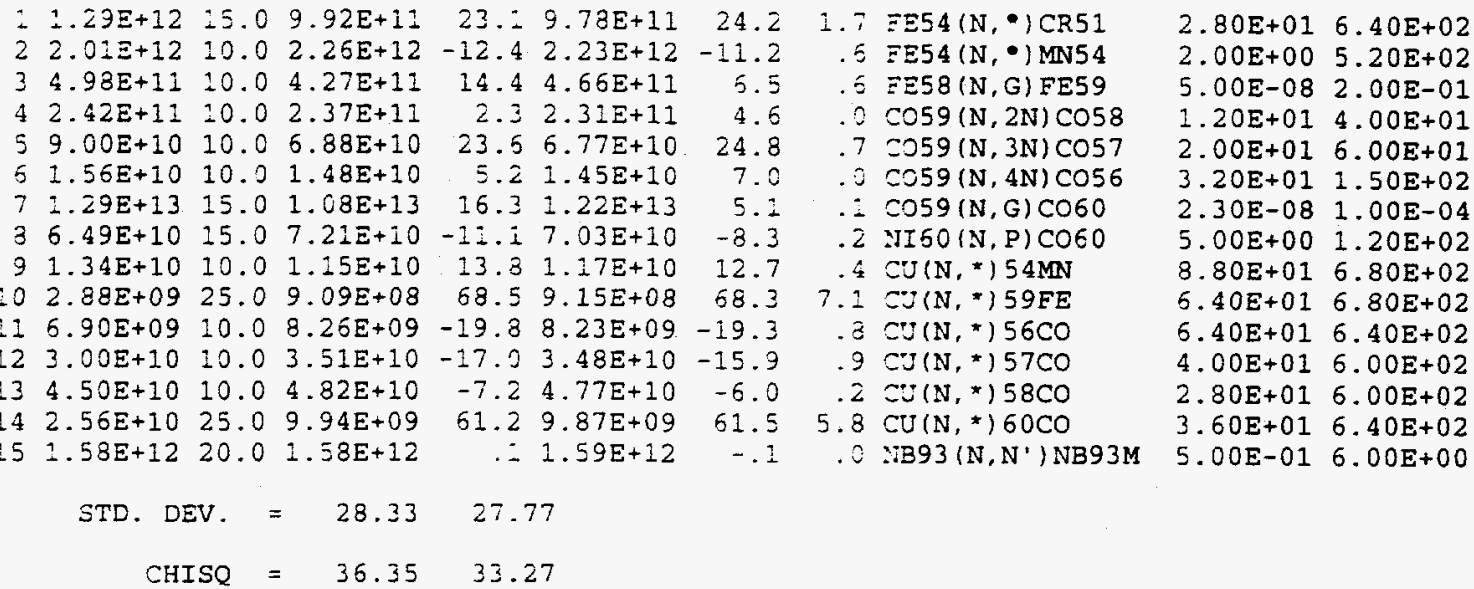

DOSIMETRY DATA INPUT CORRELATION MATRIX

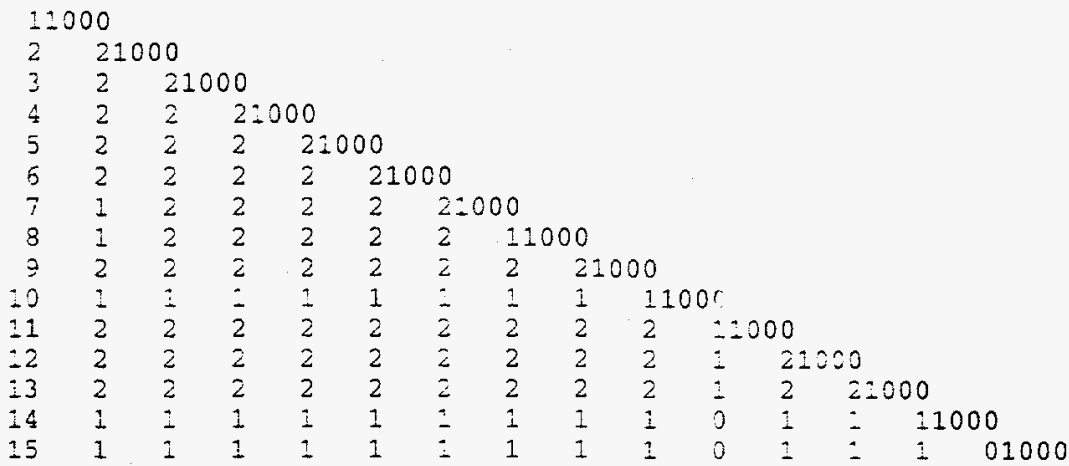

RELATIVE COV. MATRIX OF ACTIVITIES

\% CORRELATION MATRIX

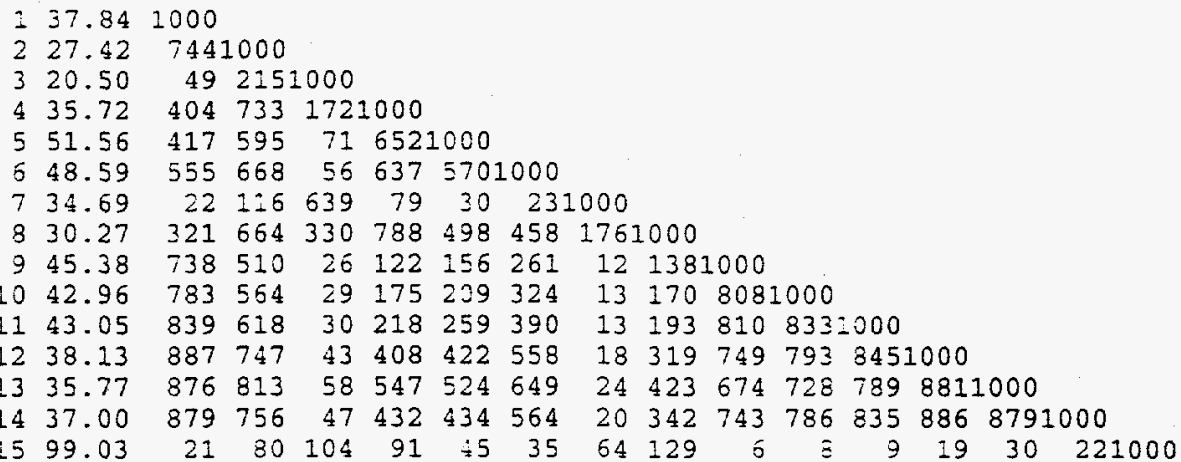

CONTRIBUTION DUE TO INPUT FLUX COV. MATRIX

8

CORREIATION MATRIX

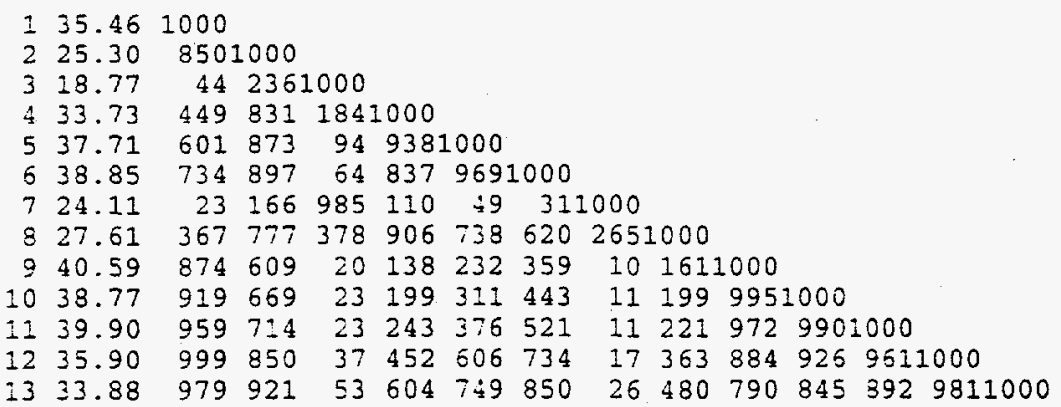


$\begin{array}{lllllllllllllll}44 & 34.61 & 996 & 866 & 42 & 482 & 628 & 748 & 20 & 391 & 682 & 924 & 955 & 999 & 9851000\end{array}$

i5 $16.13 \quad 119506 \quad 567 \quad 573 \quad 358 \quad 253 \quad 537 \quad 342 \quad 28 \quad 40 \quad 44108 \quad 175 \quad 1261000$

CONTRIBUTION DUE TO INPUT X-SEC. COV. MATRIX

CORREIATION MATRIX

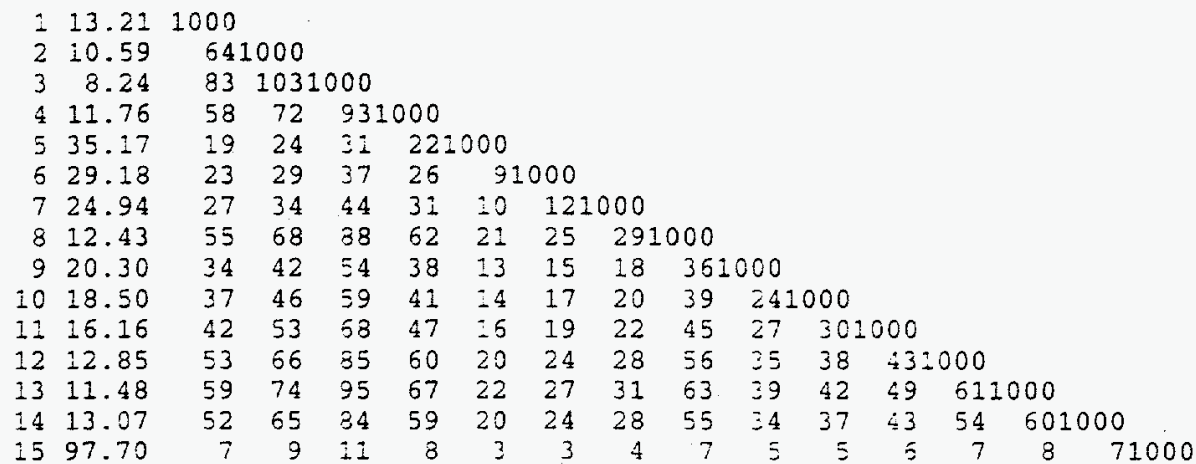

DIFEERENTIAL FLUXES INPUT NORMALIZED BY .9958

1 1. $1.000 E-10 \quad 8.227 E+17 \quad 5.583 E+17$

OE -09

1. $000 \mathrm{E}-08$

$2.300 \mathrm{E}-08$

5. $000 \mathrm{E}-08$

1. $150 \mathrm{E}-07$

2. 550E-07

5. $500 \mathrm{E}-07$

1. $275 E-06$

2. $800 \Xi-06$

6. $300 \mathrm{E}-06$

3. $301 E+17$

$2.151 E+17 \quad-.752 E+17$

1. $809 E+17$

1. $470 \mathrm{E}+17$

1. $273 E+17 \quad 2.034 E+17$

$1.105 \mathrm{E}+17 \quad 9.013 \mathrm{E}+16$

8. $881 E+167.305 E+16$

$5.498 E+16 \quad 5.395 E+16$

$4.825 E+16$

4. $046 E+16$

2. $767 E+16$

2. $344 E+16$

RATIO

ST DEV

:JEW

$\leq .231$

37.64

$\div 6.08$

OLD

RATIO

INT ELUX >+-

32.34

1.231

1. 226

1. 216

1. 205

1. 193

1. 180

1. 268

1. $919 E+1$

$.543 E+16$

$1 . \div 56$

3. $000 \mathrm{E}-05$

4. $596 \mathrm{E}+15$

$8.939 E+15$

$6.900 E-05$

3. $030 E+15 \quad 2.676 E+15$

1. 143

1.132

1. 121

i. 108

$2.800 E-04$

1. $441 \mathrm{E}+15$ 1. $286 \mathrm{E}+15$

$5.750 E-04$

$6.828 \mathrm{E}+14$

$.164 E+14$

$-.095$

2. 083

1.072

2. $000 \mathrm{E}-03$

3. $852 \mathrm{E}+14$

$3.556 E+14$

$3.400 E-03$

2. $993 E+14$

$2.791 E+14$

$5.500 E-03$

2. $329 E+14$

2. $194 \mathrm{E}+14$

9. 200E-03

1. $810 E+14$

1. $721 E+14$

1. 062

1. 052

1.043

1. $200 E-02$

$6.876 E+13$

1. $627 \mathrm{E}+1$

5. 250E-02

4. $129 E+13$

$6.659 \mathrm{E}+13$

1. 032

$\therefore .023$

1. $000 E-01$

2. $959 E+13$

$4.037 \mathrm{E}+13$

2. $000 \mathrm{E}-01$

1. $738 \mathrm{E}+13$

2. $910 E+13$

1. 017

1.012

$5.000 \mathrm{E}-01$

8. $115 \mathrm{E}+12$

$1.717 \mathrm{E}+13$

1. 009

$1.000 \mathrm{E}+00$

1. $502 \pm+1$

3. $397 E+12$

1. 007

1. 004

3. $000 E+00$

7. $568 \mathrm{E}+11$

1. $496 \mathrm{E}+12$

4. $000 E+00$

3. $611 E+11$

7. $580 E+11$

.998

.991

5. $000 E+00 \quad 2.082 E+11$

3. $643 E+11$

.985

6. $000 E+00 \quad 1.354 E+11 \quad 1.382 E+11$

$7.815 E+10 \quad 8.016 E+10$

7. $000 E+0$

6. $081 E+10$

$6.262 E+10$

.

4. $718 \mathrm{E}+10$

4. $872 E+10$

1. $000 \mathrm{E}+01$

3. $828 \mathrm{E}+10$

3. $960 \mathrm{E}+10$

1. $100 \mathrm{E}+01$

$267 E+10$

3. $383 \mathrm{E}+10$

2. $756 \mathrm{E}+10 \quad 2.854 \mathrm{E}+10$

1. $300 \mathrm{E}+01$

2. $334 \mathrm{E}+10$

2. $415 E+10$

1. $400 E+01$

2. $157 \mathrm{E}+10$

2. $229 E+10$

$21.500 E+01$

2. $044 E+10$

2. $108 E+10$

1. $700 E+01$

$1.948 \mathrm{E}+1$

2. $004 \mathrm{E}+10$

$1.913 E+10$

$51.800 \mathrm{E}+01$

1. $803 E+10$

2. $845 \mathrm{E}+10$

$1.900 \mathrm{E}+01$

1. $758 E+10$

1. $795 \mathrm{E}+10$

7 2.000E+01

1. $572 E+10$

1. $600 \mathrm{E}+10$

$92.800 E+01$

1. $179 E+10$

$1.197 \mathrm{E}+10$

.980

.975
.971

.968

.967

.966

.966

.966

.968

.970

.972

.975

.977

.980

.982

.984

30.72
29.03

$\div 3.59$

.817

$2.884 \mathrm{E}+13$

6.63

6.63

29.03

$\div 1.15$

.771

$2.883 E+13$

6.63

$\div 0.22$

.746

$2.883 E+13$

6.63

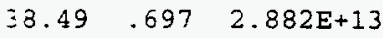

6.63

20.12

¿. 24

673

$2.880 \mathrm{E}+13$

6.63

$18.3 \hat{\jmath}$

2.00

628

2. $873 \mathrm{E}+13$

6. 64

16.75

$\div 3.10$

5.45

$\$ 4.33$

25.77

.608

2. $866 E+13$

6.65

6.65

13.52

25.25

.577

$842 E+13$

6.66

12.79

12.00

$2 \div .17$

$.5672 .825 E+13$

6.66

6.66

6.66

$11.34 \quad 20.33 \quad .558 \quad 2.752 \mathrm{E}+13$

$\pm 0.7 \equiv$

10.23

$\div .10$

.558

$752 \mathrm{E}+13$
$2.728 \mathrm{E}+13$

6.64

9.74

9.29

$\div 8.00$

.569

$2.700 E+13$

6.58

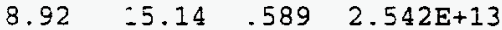

$\begin{array}{llll}7.87 & -3.20 & .596 & 2.495 \mathrm{E}+13\end{array}$

$\begin{array}{lllll}6.65 & \because .01 & .604 & 2.216 \mathrm{E}+13\end{array}$

$\begin{array}{llll}6.05 & 7.93 & .610 & 2.020 \mathrm{E}+13\end{array}$

5.52

5.26

6.84

3.98

3.54

615

1. $724 \mathrm{E}+13$

10.03

12.93

-1. 47

.616

1. $203 \mathrm{E}+13$

$-7.19$

583

$7.972 E+12$

4.44

25.73

.573

3. $049 \mathrm{E}+12$

14.64

25.77

.561

2. $292 \mathrm{E}+12$

4.58

$=7.67$

.547
.530

-. $931 E+12$

$5131.588 \mathrm{E}+12$

14. 41

$\div 9.18$

1. $588 \mathrm{E}+12$

4.14

29.83

.494

1. $449 E+12$

13.84

$\$ 0.46$

32.07

454

1. $401 \mathrm{E}+12$

13.16

12.80

12.46 


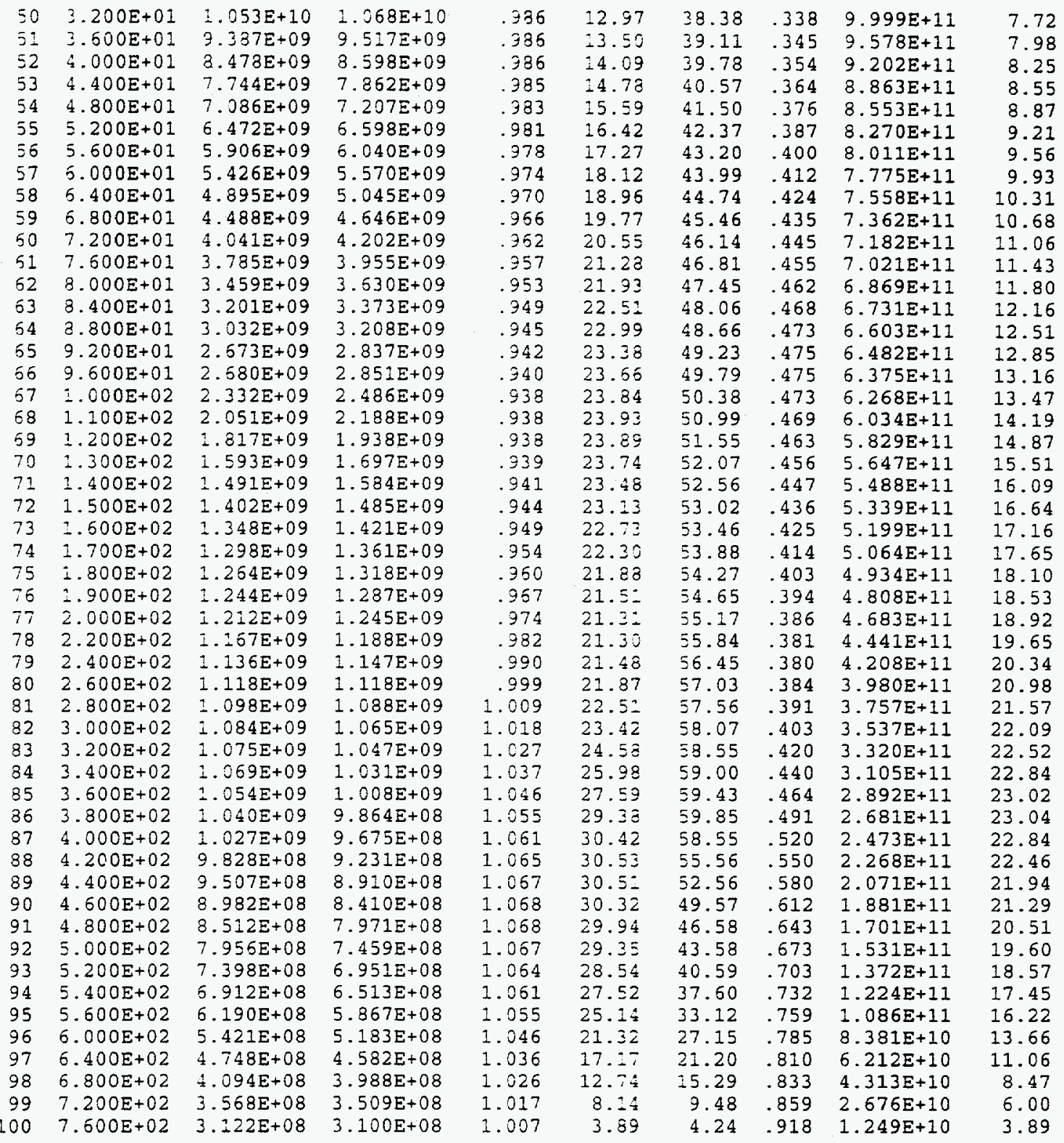

INTEGRALS OF SPECTRA

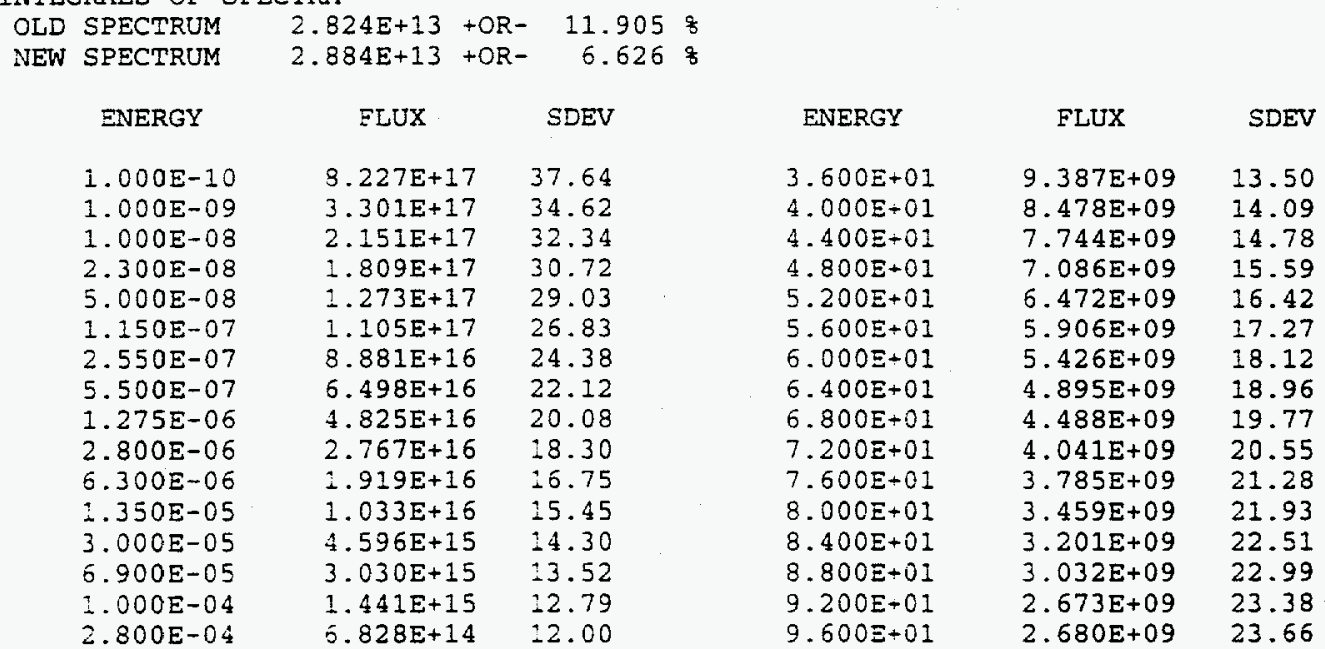




\begin{tabular}{|c|c|c|c|c|c|}
\hline $5.750 E-04$ & $3.377 \mathrm{E}+14$ & $=2.34$ & $\therefore .000 \Xi+02$ & $2.332 E+09$ & 23.84 \\
\hline $2.275 \mathrm{E}-03$ & $3.852 \mathrm{E}+14$ & $=0.75$ & $\therefore 200 \Xi+02$ & $2.051 E+09$ & 23.93 \\
\hline $2.000 E-03$ & $2.993 E+14$ & 10.23 & $\therefore 200 \Xi+02$ & $1.817 \mathrm{E}+09$ & 23.89 \\
\hline $3.400 \mathrm{E}-03$ & $2.329 E+14$ & 9.74 & $\therefore 300 E+02$ & $1.593 \mathrm{E}+09$ & 23.74 \\
\hline $5.500 E-03$ & $1.810 \mathrm{E}+14$ & 3.29 & $\therefore 400 E+02$ & 1. $491 E+09$ & 23.48 \\
\hline $9.200 E-03$ & $1.698 \mathrm{E}+14$ & 3.92 & $=.500 E+02$ & $1.402 E+09$ & 23.1 \\
\hline i. 200E-02 & $5.876 E+13$ & 7.87 & $\therefore 600 E+02$ & 1. $348 \mathrm{E}+09$ & 22.73 \\
\hline $5.250 E-02$ & 4. $129 E+13$ & 5.65 & $\therefore 700 E+02$ & I. $298 E+09$ & 22.3 \\
\hline $\pm .000 E-01$ & $2.959 E+13$ & 5.05 & $\therefore .800 E+02$ & $1.264 E+09$ & 21.8 \\
\hline $2.000 \mathrm{E}-01$ & $\pm .738 E+13$ & 5.52 & $\therefore .900 E+02$ & 1. $244 E+09$ & 21.5 \\
\hline $5.000 \mathrm{E}-01$ & 3. $115 E+12$ & 5.26 & $2.000 \bar{E}+02$ & $1.212 E+09$ & 21.3 \\
\hline $1.000 E+00$ & 3. $421 \mathrm{E}+12$ & 5.84 & $2.200 E+02$ & $1.167 E \div 09$ & 21.3 \\
\hline $2.000 E+00$ & 1. $502 \mathrm{E}+12$ & 20.03 & $2.400 \Xi+02$ & $1.136 \mathrm{E}+09$ & 21.48 \\
\hline $3.000 E+00$ & $7.568 E+11$ & \pm 2.93 & $2.500 \equiv-02$ & 1. $118 \mathrm{E}+09$ & 21.87 \\
\hline$\$ .000 E+00$ & $3.611 \mathrm{E}+11$ & $i 4.44$ & $2.800 \Xi-02$ & 1. $098 \mathrm{E}+09$ & 22.5 \\
\hline $5.000 E+00$ & $2.082 E+11$ & $\lcm{14.64}$ & $3.000 \equiv+02$ & 1. $084 \mathrm{E}+09$ & 23.4 \\
\hline $6.000 E+00$ & $1.354 E+1 I$ & 14.68 & $3.200 \equiv+02$ & $1.075 E+09$ & 24.5 \\
\hline $7.000 E+00$ & $7.815 E+10$ & \pm 4.59 & $3.400 \equiv+02$ & $1.069 E+09$ & 25.9 \\
\hline $8.000 E+00$ & $5.081 E+10$ & 14.41 & $3.600 \equiv+02$ & $1.054 \mathrm{E}+09$ & 27.5 \\
\hline $9.000 \mathrm{E}+00$ & $4.718 E+10$ & 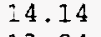 & $3.300 \equiv+02$ & $1.040 E+09$ & 29.3 \\
\hline 1.000E+01 & $3.828 E+10$ & $\div 3.84$ & $\div .000 \equiv-02$ & $1.027 E+09$ & 30.4 \\
\hline $1.100 E+01$ & $3.267 E+10$ & 13.51 & $4.200 \equiv+02$ & $9.828 E+08$ & 30.5 \\
\hline 1. $200 \mathrm{E}+01$ & $2.756 \mathrm{E}+10$ & 13.16 & $4.400 \equiv-02$ & $9.507 E+08$ & 30.5 \\
\hline 1. $300 E+01$ & $2.334 \mathrm{E}+10$ & 12.80 & $4.600 \equiv+02$ & $8.982 E+08$ & 30.3 \\
\hline $1.400 E+01$ & $2.157 \mathrm{E}+10$ & 12.46 & $4.80 C E \div 02$ & $8.512 E+08$ & 29.9 \\
\hline $1.500 E+01$ & $2.044 \mathrm{E}+10$ & \pm 2.16 & $\Xi .000 \Xi+02$ & $7.956 E+08$ & 29.3 \\
\hline $1.600 E+01$ & $1.948 E+10$ & 11.90 & $\equiv .20 c \equiv-02$ & $7.398 E+08$ & 28.5 \\
\hline 1. $700 E+01$ & $1.865 E+10$ & $\therefore .71$ & $\Xi .40 C \equiv-02$ & $6.912 E+08$ & 27.5 \\
\hline $1.800 \mathrm{E}+01$ & $1.803 E+10$ & 12.60 & $\Xi .600 \equiv-02$ & $6.190 \mathrm{E}+08$ & 25.1 \\
\hline $1.900 \mathrm{E}+01$ & 2. $758 E+10$ & 11.56 & $5.000 \equiv-02$ & $5.421 E+08$ & 21.3 \\
\hline $2.000 \mathrm{E}+01$ & 1. $572 \mathrm{E}+10$ & 1.74 & $5.400 \equiv-02$ & $4.748 E+08$ & 17.1 \\
\hline $2.400 E+01$ & 1. $348 E+10$ & $\div 2.09$ & $5.306 \equiv-02$ & $4.094 E+08$ & 12.7 \\
\hline $2.800 E+01$ & $1.179 E+10$ & 22.50 & $7.200 \equiv-02$ & $3.568 E+08$ & 8.1 \\
\hline $3.200 E+01$ & $1.053 E+10$ & 22.97 & $7.600 \equiv-02$ & $3.122 E+08$ & .8 \\
\hline
\end{tabular}

SUMMARY OF BROAD-GROUP FLUXES, FLUENCES, ANC WCERTAINTIES

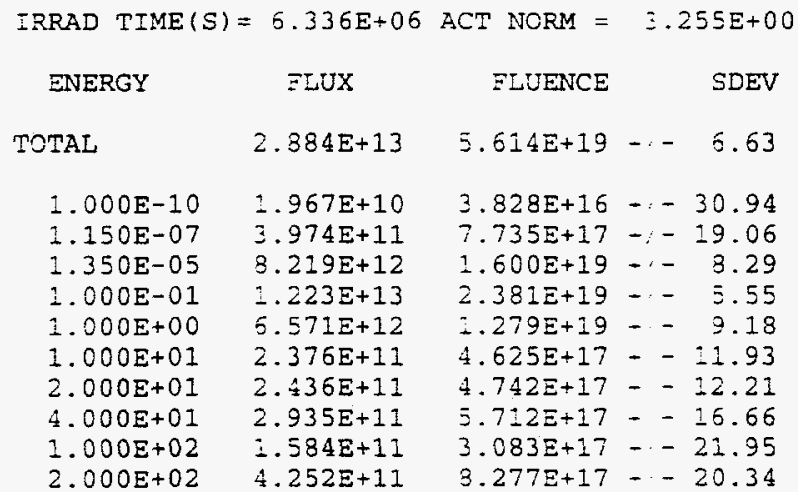

SUMMARY OF INTEGRATED GROUP ENERGY FLUXES AS:D FLUENCES

\begin{tabular}{|c|c|c|c|c|c|}
\hline $\begin{array}{l}\text { ENERGY } \\
\mathrm{MeV}\end{array}$ & $\begin{array}{c}\text { FLUX } \\
=/ \mathrm{cm} 2-\mathrm{s}-\mathrm{mA}\end{array}$ & $\begin{array}{l}=\operatorname{LUX} \\
\operatorname{2} / \mathrm{cm} 2-\mathrm{s}\end{array}$ & $\begin{array}{l}\text { FLUENCE } \\
\mathrm{n} / \mathrm{cm} 2\end{array}$ & $\begin{aligned} & \text { EAANDARD } \\
= & \text { JVIATION }\end{aligned}$ & 8 \\
\hline $\begin{array}{c}<1.0 \\
0.1-1.0 \\
1.0-10.0 \\
10.0-20.0 \\
20.0-40.0 \\
40.0-100.0 \\
100.0-200.0 \\
>200.0 \\
\text { TOTAL }\end{array}$ & $\begin{array}{l}8.636 E+12 \\
1.223 E+13 \\
6.571 E+12 \\
2.376 E+11 \\
2.436 E+11 \\
2.935 E+11 \\
1.584 E+11 \\
4.252 E+11 \\
2.884 E+13\end{array}$ & $\begin{array}{l}2.653 \mathrm{E}+12 \\
3.757 \mathrm{E}+12 \\
2.019 \mathrm{E}+12 \\
7.300 \mathrm{E}+10 \\
7.485 \mathrm{E}+10 \\
9.016 \mathrm{E}+10 \\
4.866 \mathrm{E}+10 \\
1.306 \mathrm{E}+11 \\
8.860 \mathrm{E}+12\end{array}$ & $\begin{array}{l}1.681 E+19 \\
2.381 E+19 \\
\text { 2. } 279 E+19 \\
4.625 E+17 \\
4.742 E+17 \\
5.712 E+17 \\
3.083 E+17 \\
8.277 E+17 \\
5.614 E+19\end{array}$ & $\begin{array}{l}-8.29 \\
--5.55 \\
--9.18 \\
--11.93 \\
--12.21 \\
-i-16.66 \\
--21.95 \\
--20.34 \\
-i-6.63\end{array}$ & $\begin{array}{r}29.95 \\
42.41 \\
22.78 \\
.82 \\
.84 \\
1.02 \\
.55 \\
1.47\end{array}$ \\
\hline
\end{tabular}

THE PERCENTAGE OF NEUTRONS ABOVE $20 \mathrm{MeV}=\Sigma-3.89$ 
RELATIVE COVARIANCES $(10 \times 10)$

$\begin{array}{rrrrrrrrrr}1000 & 381 & 121 & -113 & -153 & -40 & 72 & 45 & 5 & 12 \\ 500 & 459 & 124 & 23 & -28 & 53 & 50 & -5 & 15 & \\ 1000 & 390 & 793 & 237 & -108 & -51 & 57 & 53 & & \\ 500 & 943 & 402 & -132 & -119 & 58 & 51 & & & \\ 1000 & 582 & -105 & -173 & 56 & 71 & & & \\ 1000 & 517 & -103 & -128 & 92 & & & & \\ 1000 & 544 & -189 & -197 & & & & & \\ 1000 & 407 & -595 & & & & & & \end{array}$


$3-1$ ?о $3-5$

$100 \quad 12 \quad 2$

$.005020 .0000-030020.0000-.0300-3300=3550$ $.0000 \quad 0 \quad 0 \quad 5.3350 E+06$

$=554 \mathrm{PX} \quad 1.108 \mathrm{E}-13$

EE58G $7.540 E-14 \quad .10$

$\cos 92 \quad 1.577 \mathrm{E}-14 \quad .10$

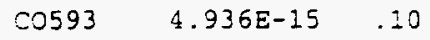

CO59G $2.190 \mathrm{E}-12 \quad .15$

CUX54MN $3.562 \mathrm{E}-16 \quad .10$

CUX59FE $1.232 \mathrm{E}-16 \quad .25$

CUX56CO $1.859 \mathrm{E}-16 \quad .10$

CUX57CO $1.022 \mathrm{E}-15 \quad .10$

CUX58CO $\quad 1.866 \mathrm{E}-15 \quad .10$

CUX60CO $1.390 E-15 \quad .25$

NB93N $1.323 E-13 \quad .20$

0 FRACTIONAL ERRORS IN INPUT SPECTRUM

$161.000 E+00$

1.000E-11 1.000E-07 1.000E-05 1.000E-03 1.000E-02:.000E-01 4.000E-01 1.000E+00 $4.000 E+00 \quad 1.000 E+012.000 E+01 \quad 4.400 E+01 \quad 1.000 E+02 \quad 4.000 E+02 \quad 8.000 E+02 \quad 0.000 E+00$ $5.000 E-01$ 4.000E-01 2.800E-01 2.000E-01 1.500E-01 ‥000E-01 8.000E-02 8.000E-02 $2.500 E-01$ 3.000E-01 3.500E-01 4.000E-01 5.000E-01 5.000E-01 $0.000 E+00 \quad 0.000 E+00$

: INPUT SPECTRUM

$1001.000 E+00$

$\begin{array}{llllllll}6.262 E+17 & 5.117 E+17 & 4.183 E+17 & 3.387 E+17 & 2.361 E+17 & =.733 E+17 & 1.118 E+17 & 6.228 E+16\end{array}$ $3.523 \mathrm{E}+16 \quad 1.784 \mathrm{E}+16 \quad 8.458 \mathrm{E}+15 \quad 4.182 \mathrm{E}+15 \quad 1.705 \mathrm{E}+15 \quad 1.246 \mathrm{E}+15 \quad 6.043 \mathrm{E}+14 \quad 3.812 \mathrm{E}+14$ $1.818 E+14 \quad 1.240 E+14 \quad 9.096 E+13 \quad 7.415 E+13 \quad 5.177 E+13 \quad 4.339 E+13 \quad 1.945 E+13 \quad 1.243 E+13$ $3.329 \mathrm{E}+12 \quad 4.720 \mathrm{E}+12 \quad 1.942 \mathrm{E}+12 \quad 8.184 \mathrm{E}+11 \quad 3.996 \mathrm{E}+11 \quad \overline{2} .295 \mathrm{E}+11 \quad 1.320 \mathrm{E}+11 \quad 7.717 \mathrm{E}+10$ $4.143 E+102.639 E+10 \quad 2.009 E+10 \quad 1.598 E+10 \quad 1.256 E+10 \quad-.351 E+10 \quad 8.433 E+09 \quad 6.818 E+09$ $5.840 E+09 \quad 5.026 E+09 \quad 4.340 E+09 \quad 3.911 E+09 \quad 3.679 E+09 \quad 3.477 E+09 \quad 3.065 E+09 \quad 2.511 E+09$ $2.187 E+09 \quad 1.871 E+09 \quad 1.616 E+09 \quad 1.408 E+09 \quad 1.233 E+09 \quad \therefore .284 E+09 \quad 9.565 E+08 \quad 8.180 E+08$ $\begin{array}{llllllll}7.125 E+08 & 6.221 E+08 & 5.258 E+08 & 4.692 E+08 & 4.197 E+08 & 3.702 E+08 & 3.334 E+08 & 3.014 E+08\end{array}$ $2.737 \mathrm{E}+08 \quad 2.541 \mathrm{E}+08 \quad 2.265 \mathrm{E}+08 \quad 1.984 \mathrm{E}+08 \quad 1.788 \mathrm{E}+08 \quad 2.559 \mathrm{E}+08 \quad 1.476 \mathrm{E}+08 \quad 1.396 \mathrm{E}+08$ $\begin{array}{llllllll}1.332 E+08 & 1.238 E+08 & 1.240 E+08 & 1.147 E+08 & 1.076 E+08 & 9.950 E+07 & 9.442 E+07 & 8.723 E+07\end{array}$ $\begin{array}{llllllll}8.675 E+07 & 8.381 E+07 & 8.262 E+07 & 8.164 E+07 & 7.807 E+07 & 7.473 E+07 & 7.243 E+07 & 6.853 E+07\end{array}$ $\begin{array}{llllllll}5.470 E+07 & 5.203 E+07 & 5.836 E+07 & 5.576 E+07 & 5.231 E+07 & \therefore .938 E+07 \quad 4.559 E+07 \quad 4.007 E+07\end{array}$ $3.656 \mathrm{E}+07 \quad 3.290 \mathrm{E}+07 \quad 3.022 \mathrm{E}+07 \quad 2.786 \mathrm{E}+07$

\begin{tabular}{|c|c|c|c|c|c|}
\hline \multirow{3}{*}{ 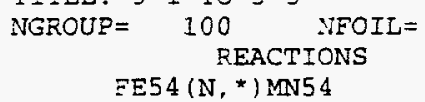 } & 12 & ACTZVITY NORM= & \multicolumn{3}{|c|}{$3.255 E+00$} \\
\hline & COVER & $\therefore$ CTIVITIES & & $E-S H I E$ & DING \\
\hline & .00 & $3.607 \mathrm{E}-13$ & & & 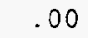 \\
\hline EE58 (N, G)FE59 & .00 & $2.454 \mathrm{E}-13$ & $I F X$ & EENG & 4.7 \\
\hline $\cos 9(\mathrm{~N}, 2 \mathrm{~N}) \cos 8$ & .00 & $\equiv .133 \mathrm{E}-14$ & & & .0 \\
\hline $\cos 9(\mathrm{~N}, 3 \mathrm{~N}) \cos 7$ & .00 & $\therefore 607 E-14$ & & & .0 \\
\hline $\cos 9(\mathrm{~N}, \mathrm{G}) \operatorname{co6} 0$ & .00 & $. .128 \mathrm{E}-12$ & IFX & CONG & 1.9 \\
\hline$C U(N, *) 54 \mathrm{MN}$ & .00 & $\therefore 159 \mathrm{E}-15$ & & & .0 \\
\hline $\mathrm{CU}(\mathrm{N}, *) 59 \mathrm{FE}$ & .00 & $\therefore .010 \mathrm{E}-16$ & & & .0 \\
\hline $\mathrm{CU}\left(\mathrm{N},{ }^{*}\right) 56 \mathrm{CO}$ & .00 & $5.051 \mathrm{E}-16$ & & & .0 \\
\hline $\mathrm{CU}\left(\mathrm{N},{ }^{*}\right) 57 \mathrm{CO}$ & .00 & $3.327 \mathrm{E}-15$ & & & .0 \\
\hline $\mathrm{CU}(\mathrm{N}, *) 58 \mathrm{CO}$ & .00 & $=.074 \mathrm{E}-15$ & & & .0 \\
\hline $\mathrm{CU}(\mathrm{N}, *) 60 \mathrm{CO}$ & .00 & $\div .524 \mathrm{E}-15$ & & & .00 \\
\hline $\mathrm{NB9} 3\left(\mathrm{~N}, \mathrm{~N}^{\prime}\right) \mathrm{NB} 93 \mathrm{M}$ & .00 & $\therefore 306 \mathrm{E}-13$ & & & 0 \\
\hline
\end{tabular}

COVARIANCE PARAMETERS

FLUXES: WIDTH $=2.0000 E+01$ ECOV $=3.0000 \mathrm{E}-02$

SIGMAS: WIDTH $=2.0000 \mathrm{E}+01$ XCOV $=3.0000 \mathrm{E}-02$

ACTIVITIES $=5.0000 \mathrm{E}-03$ CROSS SECTIONS $=3.2200 \mathrm{E}-02$

DETERM $=7.409 * 10 * *-18.00 \quad$ ERROR $=\quad 1.034 \mathrm{E}-15$

INPUT NORMALIZATION DATA

$\mathrm{AK} 1=\quad .0000$ VAK $=\quad .00090 \mathrm{NORM}=0 \mathrm{RENORM}=\quad .3918 \mathrm{CHI} 2=23.9212$

\begin{tabular}{|c|c|c|c|c|c|c|c|c|c|}
\hline MEASURED & $+1-\frac{3}{8}$ & BEFORE & DIE\& & AFTER & DIE & CHI & ミEACTION & 90 \% & LIMITS \\
\hline 3. $61 E+11$ & 10. C & $4.24 E+11$ & -17.7 & $4.08 \mathrm{E}+11$ & -13.2 & 1.1 & $\equiv \equiv 54(\mathrm{~N}, \star) \mathrm{MN5} 4$ & $2.00 E+00$ & $3.60 \mathrm{E}+02$ \\
\hline $22.45 E+11$ & 10.0 & $2.41 E+11$ & 1.9 & $2.42 \mathrm{E}+11$ & 1.2 & .0 & $\equiv \equiv 58(\mathrm{~N}, \mathrm{G})$ EES9 & $1.00 E-09$ & $5.25 \mathrm{E}-02$ \\
\hline $35.13 E+10$ & 10.0 & $4.99 E+10$ & 2.7 & $4.98 \mathrm{E}+10$ & 3.0 & .0 & $\approx 259(\mathrm{~N}, 2 \mathrm{~N}) \cos 8$ & $1.20 \mathrm{E}+01$ & 3. $60 \mathrm{E}+01$ \\
\hline $41.61 E+10$ & 10.0 & $1.16 E+10$ & 28.1 & 1. $19 \mathrm{E}+10$ & 25.9 & .9 & $\operatorname{C259}(\mathrm{N}, 3 \mathrm{~N}) \operatorname{Co5} 7$ & $2.00 E+01$ & $5.20 E+01$ \\
\hline $57.13 E+12$ & 15.0 & $6.70 E+12$ & 6.0 & $6.82 \mathrm{E}+12$ & 4.3 & .0 & $==59(\mathrm{~N}, \mathrm{G}) \mathrm{CO} 60$ & 1. $00 E-09$ & $1.00 \mathrm{E}-04$ \\
\hline $61.16 E+09$ & 10.0 & $9.55 \Xi+08$ & 17.6 & $9.75 E+08$ & 15.9 & .8 & $\because \ddot{U}\left(\mathrm{~N},{ }^{*}\right) 54 \mathrm{MN}$ & $7.20 \mathrm{E}+01$ & $6.80 \mathrm{E}+02$ \\
\hline $74.01 E+08$ & 25.0 & $7.82 \mathrm{E}+07$ & 80.5 & $7.93 \mathrm{E}+07$ & 80.2 & 10.2 & $\because(\mathrm{N}, *) 59 \mathrm{FE}$ & $5.60 \mathrm{E}+01$ & $6.80 \mathrm{E}+02$ \\
\hline $6.05 E+08$ & 10.0 & $7.25 E+08$ & -19.7 & $7.24 \mathrm{E}+08$ & -19.6 & 1.0 & $\because(\mathrm{N}, *) 56 \mathrm{CO}$ & $6.00 \mathrm{E}+01$ & $6.40 \mathrm{E}+02$ \\
\hline $3.33 \mathrm{E}+09$ & 10.0 & $3.61 E+09$ & -8.7 & $3.64 \mathrm{E}+09$ & -9.3 & .3 & $\mathrm{U}(\mathrm{N}, *) 57 \mathrm{CO}$ & $4.00 \mathrm{E}+01$ & $6.00 \mathrm{E}+02$ \\
\hline
\end{tabular}


$=26.07 E+09 \quad 10.0 \quad 5.64 E+09$

$\because 4.52 E+0925.0 \div .05 E+09$

$224.31 E+1120.0 \quad 4.28 E+11$
$7.2 \equiv .71 \mathrm{E}+09$

$76.71 .07 \mathrm{E}+09$

$74.00 E+11$

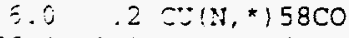

$75.49 .360(\mathrm{~N}, *) 60 \mathrm{CO}$

$.0 \mathrm{NB93}\left(\mathrm{N}, \mathrm{N}^{\circ}\right) \mathrm{NB} 93 \mathrm{M}$

STD. ZEV. = $35.20 \quad 35.63$

CHISQ $=39.3236 .62$

DOSIMETRY DATA INPUT CORRELATION MATRIX

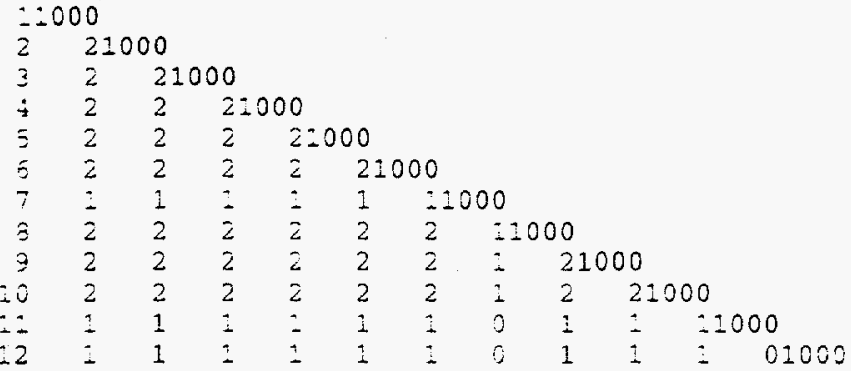

RELATIVE COV. MATRIX OF ACTIVITIES

$$
\text { * CORRELATION MATRIX }
$$

125.311000

224.822481000

$335.40 \quad 817 \quad 1221000$

$452.47 \quad 587 \quad 46 \quad 6351000$

$\begin{array}{llllll}5 & 35.64 & 165 & 739 & 72 & 251000\end{array}$

$\begin{array}{lllllll}6 & 44.23 & 332 & 18 & 135 & 168 & 121000\end{array}$

$\begin{array}{llllllll}7 & 41.45 & 399 & 21 & 205 & 237 & 13 & 8181000\end{array}$

$\begin{array}{lllllllll}3 & 42.12 & 440 & 21 & 240 & 281 & 13 & 818 & 8491000\end{array}$

$\begin{array}{llllllllll}9 & 37.05 & 628 & 32 & 490 & 492 & 19 & 709 & 776 & 8331000\end{array}$

$\begin{array}{lllllllllll}10 & 35.46 & 724 & 43 & 647 & 598 & 25 & 590 & 671 & 733 & 8911000\end{array}$

$\begin{array}{llllllllllll}11 & 35.82 & 656 & 36 & 535 & 518 & 21 & 688 & 756 & 810 & 508 & 897: 200\end{array}$

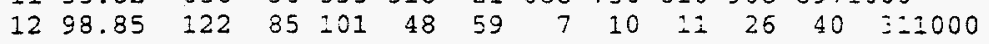

CONTRIBUTION DUE TO INPUT FLUX COV. MATRIX

3 CORRELATION MATRIX

123.541000

$233.66 \quad 2631000$

$333.37 \quad 920 \quad 1251000$

$4 \quad 37.80 \quad 865 \quad 55 \quad 9281000$

$5 \quad 27.34 \quad 217 \quad 997 \quad 90 \quad 371000$

$\begin{array}{lllllll}6 & 39.81 & 387 & 12 & 152 & 253 & 91000\end{array}$

$\begin{array}{llllllll}7 & 37.76 & 461 & 14 & 232 & 355 & 10 & 9911000\end{array}$

$\begin{array}{lllllllll}8 & 39.39 & 496 & 13 & 255 & 412 & 10 & 966 & 9901000\end{array}$

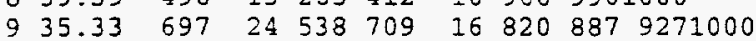

$\begin{array}{lllllllllll}10 & 33.91 & 803 & 36 & 710 & 860 & 24 & 678 & 763 & 813 & 9691000\end{array}$

$\begin{array}{llllllllllll}11 & 33.95 & 733 & 29 & 591 & 752 & 19 & 800 & 869 & 907 & 997 & 9821200\end{array}$

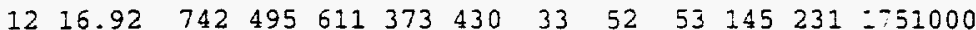

CONTRIBUTION DUE TO INPUT X-SEC. COV. MATRIX

CORRELATION MATRIX

$$
\begin{aligned}
& \text { I } 9.291000 \\
& 2 \quad 7.50 \quad 1291000 \\
& \begin{array}{lllll}
4 & 36.40 & 27 & 33 & 211000
\end{array} \\
& \begin{array}{lllllll}
6 & 19.26 & 50 & 62 & 40 & 13 & 201000
\end{array} \\
& \begin{array}{lllllllll}
8 & 14.91 & 65 & 81 & 51 & 17 & 26 & 31 & 351000
\end{array}
\end{aligned}
$$

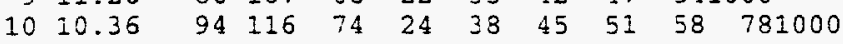

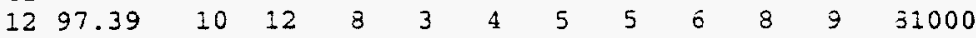$$
311.80 \quad 821021000
$$

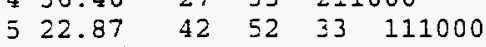$$
\begin{array}{llllllll}
7 & 17.10 & 57 & 70 & 45 & 14 & 23 & 271000
\end{array}
$$$$
\begin{array}{llllllllll}
9 & 11.20 & 86 & 107 & 68 & 22 & 35 & 42 & 47 & 541000
\end{array}
$$$$
\begin{array}{llllllllllll}
11 & 11.43 & 85 & 105 & 67 & 22 & 34 & 41 & 46 & 53 & 70 & 761230
\end{array}
$$

DIFFERENTIAL FLUXES INPUT NORMALIZED BY .9918

$\begin{array}{cccccccccc}\text { G } & \text { ENERGY } & \text { NEW } & \text { OLD } & \text { RATIO } & \text { STD DEV } & \text { NEW } & \text { OLD } & \text { RATIO } & \text { INT FLUX } \\ & 1.000 E-10 & 6.802 \mathrm{E}+17 & 6.210 \mathrm{E}+17 & 1.095 & 30.15 & 46.08 & .654 & 7.730 \mathrm{E}+12 & 7.08 \\ 2 & 1.000 \mathrm{E}-09 & 5.527 \mathrm{E}+17 & 5.075 \mathrm{E}+17 & 1.089 & 27.12 & 43.59 & .622 & 7.730 \mathrm{E}+12 & 7.08 \\ 3 & 1.000 \mathrm{E}-08 & 4.496 \mathrm{E}+17 & 4.149 \mathrm{E}+17 & 1.084 & 24.77 & 41.96 & .590 & 7.725 \mathrm{E}+12 & 7.09 \\ 4 & 2.300 \mathrm{E}-08 & 3.625 \mathrm{E}+17 & 3.359 \mathrm{E}+17 & 1.079 & 23.03 & 11.15 & .560 & 7.719 \mathrm{E}+12 & 7.09\end{array}$




\begin{tabular}{|c|c|c|c|c|c|c|c|c|}
\hline $5.000 \mathrm{E}-08$ & $2.513 \Xi+17$ & $2.342 E+17$ & $\therefore .073$ & 22.35 & 40.22 & .531 & $7.709 E+12$ & 7.11 \\
\hline $.150 \mathrm{E}-07$ & $\therefore .331 E+17$ & $\therefore .719 E+17$ & 1.065 & 19.44 & 38.49 & .505 & $7.693 E+12$ & 7.12 \\
\hline $.550 \mathrm{E}-07$ & $\therefore .170 \mathrm{E}+17$ & $\therefore .109 E+17$ & 1.056 & 27.51 & 36.24 & .483 & $7.667 \mathrm{E}+12$ & 7.15 \\
\hline 00E-07 & $5.461 E+16$ & $5.177 E+16$ & 1.046 & 15.87 & 34.06 & .466 & $7.632 E+12$ & 7.18 \\
\hline $275 E-06$ & $3.621 E+16$ & $3.494 \mathrm{E}+16$ & 1.036 & 14.54 & 32.00 & .454 & $7.586 \mathrm{E}+12$ & 7 \\
\hline $2.800 E-06$ & $\therefore .817 E+16$ & $\therefore .769 \mathrm{E}+16$ & 1.027 & 13.50 & 30.10 & .449 & $7.530 \mathrm{E}+12$ & 7.2 \\
\hline $5.300 E-06$ & $3.538 \mathrm{E}+15$ & $3.388 E+15$ & 1.018 & 12.73 & 28.35 & .449 & $7.467 \mathrm{E}+12$ & 7.27 \\
\hline $.350 E-05$ & $4.185 E+15$ & $4.148 E+15$ & 1.009 & 12.17 & 26.77 & .455 & $7.405 E+12$ & \\
\hline $.000 E-05$ & $1.692 \mathrm{E}+15$ & $=.691 E+15$ & 1.001 & 11.75 & 25.25 & .465 & $7.336 E+12$ & \\
\hline $6.900 E-05$ & i. $030 E+15$ & $2.037 E+15$ & .993 & 11.59 & 24.17 & .480 & $7.270 E+12$ & \\
\hline $1.000 \mathrm{E}-04$ & $5.906 E+14$ & $5.993 E+14$ & .985 & 11.43 & 22.99 & .497 & $7.238 E+12$ & \\
\hline $2.800 E-04$ & $3.701 E+14$ & $3.781 E+14$ & .979 & 11.13 & 21.59 & .516 & $7.132 E+12$ & \\
\hline $5.750 E-04$ & $1.754 \mathrm{E}+14$ & $\therefore .803 E+14$ & .973 & 10.87 & 20.33 & .535 & $7.023 E+12$ & 7.29 \\
\hline $1.275 E-03$ & $1.190 E+14$ & $\therefore .230 \mathrm{E}+14$ & .968 & 10.58 & 13.10 & .554 & $6.900 E+12$ & 7. \\
\hline $2.000 E-03$ & $3.694 E+13$ & $9.021 E+13$ & .964 & 10.29 & 18.00 & .572 & $5.814 \mathrm{E}+12$ & \\
\hline $.400 E-03$ & $7.062 \mathrm{E}+13$ & $7.354 E+13$ & .960 & 9.96 & $\lcm{-6.92}$ & .589 & $5.692 \mathrm{E}+12$ & \\
\hline $5.500 E-03$ & $4.917 E+13$ & $5.134 \mathrm{E}+13$ & .958 & 9.51 & 15.94 & .603 & $5.544 \mathrm{E}+12$ & 7.18 \\
\hline $9.200 E-03$ & $4.586 E+13$ & $4.799 E+13$ & .956 & 9.31 & $\$ 5.14$ & .615 & $6.362 \mathrm{E}+12$ & \\
\hline $1.200 \mathrm{E}-02$ & $1.848 E+13$ & $1.929 E+13$ & .958 & 8.26 & 13.20 & .626 & $6.233 \mathrm{E}+12$ & \\
\hline $5.250 E-02$ & 1. $187 \mathrm{E}+13$ & i. $233 \mathrm{E}+13$ & .963 & 7.00 & 11.01 & 536 & $5.485 E+12$ & \\
\hline $1.000 \mathrm{E}-01$ & $7.972 E+12$ & $8.260 E+12$ & .965 & 6.39 & 9.93 & 643 & $4.921 E+12$ & 7.02 \\
\hline $2.000 E-01$ & $4.529 E+12$ & $4.681 E+12$ & .968 & 5.83 & 8.98 & 649 & $4.124 E+12$ & 7.18 \\
\hline $5.000 E-01$ & $1.865 E+12$ & 1. $926 E+12$ & .969 & 5.56 & 8.54 & 650 & $2.765 E+12$ & \\
\hline $1.000 E+00$ & $7.760 E+11$ & $8.117 E+11$ & .956 & 7.26 & $\therefore 1.47$ & 533 & $1.832 E+12$ & 9.29 \\
\hline $2.000 E+00$ & $3.697 E+11$ & $3.963 E+11$ & .933 & 10.68 & 17.19 & 621 & $1.056 \mathrm{E}+12$ & 10.96 \\
\hline $3.000 E+00$ & $2.077 E+11$ & $2.276 E+11$ & .913 & 13.78 & 22.56 & .611 & $5.867 \mathrm{E}+11$ & 1.30 \\
\hline $.000 E+00$ & 2. $182 E+11$ & $=.309 \mathrm{E}+11$ & .903 & 15.42 & 25.73 & 599 & $4.789 \mathrm{E}+11$ & 10. \\
\hline $5.000 \mathrm{E}+00$ & $5.908 E+10$ & $7.653 E+10$ & .903 & 15.66 & 26.77 & 585 & $3.608 \mathrm{E}+11$ & 9.33 \\
\hline $6.000 E+00$ & $3.717 E+10$ & $4.109 \mathrm{E}+10$ & .905 & & 27.67 & 568 & $2.917 E+11$ & 8. \\
\hline $7.000 \mathrm{E}+00$ & $2.378 E+10$ & $2.517 E+10$ & .908 & 15.67 & 28.47 & 551 & $2.545 E+11$ & \\
\hline $.000 E+00$ & $=.821 E+10$ & $=.992 E+10$ & .914 & 15.50 & 29.18 & 531 & $2.307 E+11$ & 7. \\
\hline $9.000 E+00$ & 1. $460 E+10$ & $=.585 E+10$ & .921 & 15.24 & 29.83 & 511 & $2.125 E+11$ & 7.38 \\
\hline $1.000 E+01$ & $1.157 E+10$ & I. $246 \mathrm{E}+10$ & .929 & 14.93 & 30.46 & 490 & $1.979 E+11$ & 7.30 \\
\hline $1.100 \mathrm{E}+01$ & $9.780 E+09$ & $1.042 E+10$ & .938 & 14.57 & 31.07 & .469 & $1.864 \mathrm{E}+11$ & 7.29 \\
\hline $1.200 \mathrm{E}+01$ & $7.930 E+09$ & $8.364 E+09$ & .948 & 14.17 & 31.65 & .448 & 1. $766 \mathrm{E}+11$ & 7.31 \\
\hline $1.300 E+01$ & $5.482 E+09$ & $6.762 E+09$ & .959 & 13.75 & 32.19 & 427 & $1.687 \mathrm{E}+11$ & 7.36 \\
\hline 1. $400 \mathrm{E}+01$ & $5.614 E+09$ & $5.792 E+09$ & 969 & 13.34 & 32.70 & 408 & $1.622 \mathrm{E}+11$ & 7.42 \\
\hline $.500 E+01$ & $4.884 E+09$ & $4.985 E+09$ & .980 & 12.94 & 33.19 & 390 & 1. $566 E+11$ & 7.47 \\
\hline $1.600 \mathrm{E}+01$ & $4.262 E+09$ & $4.304 \mathrm{E}+09$ & .990 & 12.59 & 33.65 & .374 & $1.517 E+11$ & 7.52 \\
\hline $1.700 \mathrm{E}+01$ & $3.878 E+09$ & $3.879 E+09$ & 1.000 & 12.31 & 34.09 & 361 & $1.4745+11$ & 7.56 \\
\hline $1.800 E+01$ & $3.680 E+09$ & $3.649 E+09$ & 1.009 & 12.11 & 34.51 & .351 & $E+11$ & \\
\hline $1.900 E+01$ & $3.505 \equiv+09$ & $3.448 E+09$ & 1.016 & 12.00 & 34.91 & .344 & $1.399 \mathrm{E}+11$ & 7.61 \\
\hline $2.000 E+01$ & $3.110 \mathrm{E}+09$ & $3.040 \mathrm{E}+09$ & 1.023 & 12.13 & 35.66 & .340 & $1.363 E+11$ & 7.62 \\
\hline $2.400 E+01$ & $2.561 E+09$ & $2.490 E+09$ & 1.029 & 12.46 & 36.68 & .340 & 1.23 & 7.66 \\
\hline $2.800 E+01$ & $2.240 E+09$ & $2.169 E+09$ & 1.033 & 12.86 & 37.58 & .342 & $1.137 \mathrm{E}+11$ & 7.75 \\
\hline $3.200 E+0 I$ & $1.920 E+09$ & $1.856 \mathrm{E}+09$ & 1.035 & 13.34 & 38.38 & .347 & $1.047 \mathrm{E}+11$ & 7.88 \\
\hline $3.600 E+01$ & $1.660 \mathrm{E}+09$ & 1. $603 \mathrm{E}+09$ & 1.036 & 13.88 & 39.11 & .355 & $9.702 \mathrm{E}+10$ & 8. \\
\hline$E+01$ & $1.444 \mathrm{E}+09$ & $1.396 \mathrm{E}+09$ & 1.034 & 14.49 & 39.78 & .364 & $9.038 \mathrm{E}+10$ & 8.34 \\
\hline $4.400 E+01$ & $1.261 \mathrm{E}+09$ & 1. $223 \mathrm{E}+09$ & 1.032 & 15.20 & 40.57 & .375 & $8.461 E+10$ & 8.66 \\
\hline $4.800 E+01$ & $1.104 \mathrm{E}+09$ & $1.075 E+09$ & 1.027 & 16.01 & 41.50 & .386 & $7.956 \mathrm{E}+10$ & 9.05 \\
\hline $5.200 E+01$ & $9.690 E+08$ & $9.486 E+08$ & 1.021 & 16.84 & 42.37 & .398 & $7.514 \mathrm{E}+10$ & 0 \\
\hline +01 & $8.228 E+08$ & $8.113 E+08$ & 1.014 & 17.67 & 43.20 & .409 & $7.127 \mathrm{E}+10$ & 10.01 \\
\hline $6.000 E+01$ & $7.107 E+08$ & $7.066 E+08$ & 1.005 & 18.49 & 43.99 & .420 & $6.798 \mathrm{E}+10$ & 10.53 \\
\hline $6.400 \mathrm{E}+01$ & $5.147 E+08$ & $6.170 E+08$ & .996 & 19.29 & 44.74 & .431 & $6.513 \mathrm{E}+10$ & 11.07 \\
\hline $6.800 \mathrm{E}+01$ & $5.143 E+08$ & $5.215 E+08$ & .986 & 20.04 & 45.46 & .441 & $\varepsilon+10$ & 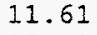 \\
\hline $7.200 E+01$ & $4.542 E+08$ & $4.653 E+08$ & .976 & 20.75 & 46.14 & .450 & $6.062 E+10$ & 12.12 \\
\hline $7.600 E+01$ & $4.019 E+08$ & $4.162 E+08$ & .966 & 21.40 & 46.81 & .457 & $5.880 E+10$ & 12.62 \\
\hline $8.000 \mathrm{E}+01$ & $3.508 \mathrm{E}+08$ & $.671 E+08$ & 95 & 21.9 & 47.45 & 463 & $E+10$ & 3.09 \\
\hline+01 & $3.128 E+08$ & $3.307 E+08$ & .946 & 22.49 & 48.06 & 468 & $5.579 E+10$ & 13.53 \\
\hline $8.800 E+01$ & $2.802 E+08$ & $2.989 E+08$ & .937 & 22.92 & 48.66 & 471 & $5.454 E+10$ & 13.94 \\
\hline $.200 E+01$ & $2.524 E+08$ & $2.714 E+08$ & .930 & 23.25 & 49.23 & 472 & $2 E+10$ & 4.3 \\
\hline $.500 E+01$ & 2.32 & $E+08$ & 924 & 23.51 & 49.79 & .472 & $E+10$ & 14.67 \\
\hline $1.000 E+02$ & $2.065 E+08$ & $2.246 E+08$ & 919 & 23.69 & $\equiv 0.38$ & .470 & $E+10$ & 15.00 \\
\hline $1.100 E+02$ & i. $802 E+08$ & $.968 E+08$ & .916 & 23.80 & 50.99 & 467 & $4.941 E+10$ & \\
\hline $.200 E+02$ & $1.622 E+08$ & $1.773 E+08$ & .91 & 23.81 & 51.55 & .462 & $4.761 E+10$ & .45 \\
\hline$E+02$ & $1.506 E+08$ & $1.645 E+08$ & .915 & 23.74 & $\Xi 2.07$ & .456 & $4.599 E+10$ & 17.11 \\
\hline 1. $400 E+02$ & $1.344 E+08$ & $1.464 \mathrm{E}+08$ & .918 & 23.59 & 52.56 & .449 & $4.448 \mathrm{E}+10$ & 17. \\
\hline $.500 \mathrm{E}+02$ & $1.277 E+08$ & $2.384 \mathrm{E}+08$ & .922 & 23.39 & 53.02 & .441 & $4.314 \mathrm{E}+10$ & 18.32 \\
\hline+02 & $1.227 E+08$ & $1.321 E+08$ & .928 & 23.17 & 53.46 & .433 & $4.186 \mathrm{E}+10$ & 18.87 \\
\hline $1.700 \mathrm{E}+02$ & $1.150 \mathrm{E}+08$ & $2.228 \mathrm{E}+08$ & .936 & 22.96 & $\Xi 3.88$ & .426 & $4.063 E+10$ & 19.3 \\
\hline $.800 \mathrm{E}+02$ & $1.163 E+08$ & $1.230 \mathrm{E}+08$ & .946 & 22.78 & 54.27 & .420 & $3.949 E+10$ & 19.85 \\
\hline $1.900 \mathrm{E}+02$ & $1.088 E+08$ & $2.138 E+08$ & .956 & 22.67 & $\$ 4.65$ & .415 & $3.832 E+10$ & 20.3 \\
\hline $2.000 \mathrm{E}+02$ & $1.033 E+08$ & $1.067 E+08$ & .968 & 22.75 & $\Xi 5.17$ & .412 & $3.723 E+10$ & 20.6 \\
\hline $2.200 E+02$ & $9.676 \mathrm{E}+07$ & $9.868 \mathrm{E}+07$ & .981 & 23.03 & 55.84 & .413 & $3.517 \mathrm{E}+10$ & 21.40 \\
\hline $2.400 E+02$ & $9.308 E+07$ & $9.364 \mathrm{E}+07$ & .994 & 23.49 & 56.45 & .416 & $3.323 \mathrm{E}+10$ & 22.0 \\
\hline $2.600 E+02$ & $8.721 E+07$ & $8.651 E+07$ & 1.008 & 24.13 & 57.03 & .423 & $3.137 \mathrm{E}+10$ & 22.62 \\
\hline $2.800 E+02$ & $8.796 E+07$ & $8.604 E+07$ & 1.022 & 24.98 & 57.56 & .434 & $2.963 E+10$ & 23.1 \\
\hline $3.000 E+02$ & $8.618 E+07$ & $8.312 E+07$ & 1.037 & 26.04 & 58.07 & 448 & $2.787 E+10$ & 23. \\
\hline
\end{tabular}




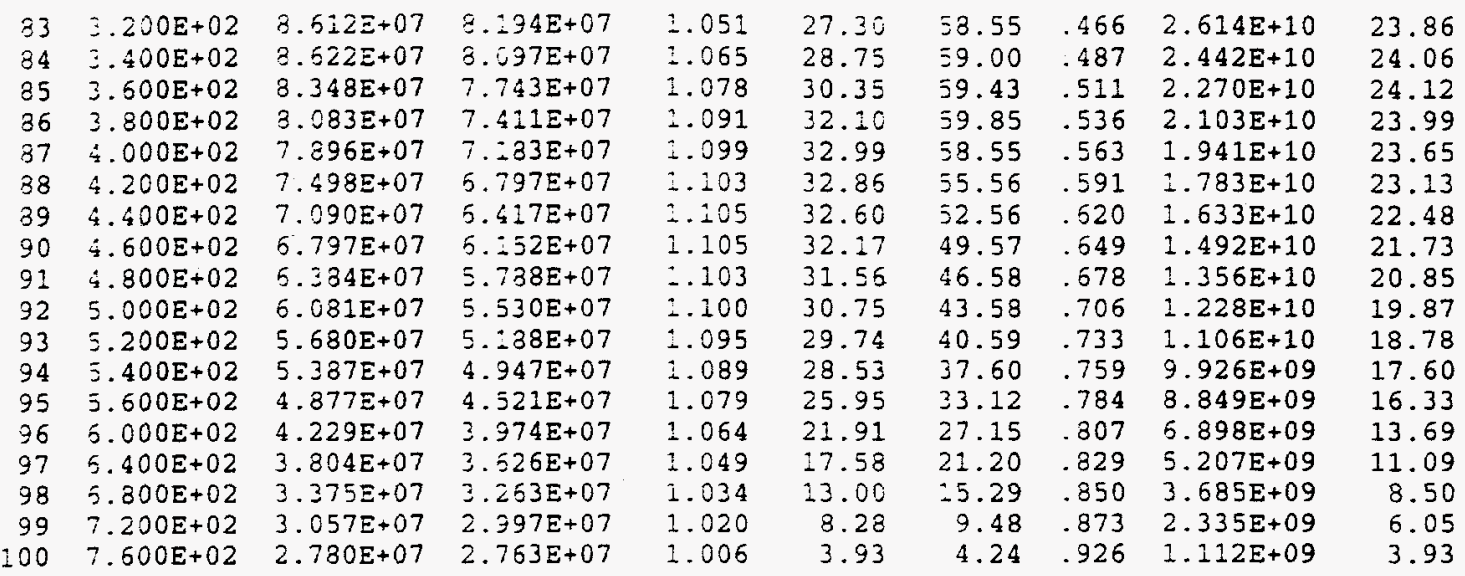

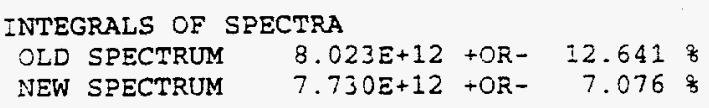

\begin{tabular}{|c|c|c|c|c|c|}
\hline ENERGY & ELuX & SDEV & ENERGY & ELUX & SDEV \\
\hline $1.000 E-10$ & $5.802 E+17$ & 30.15 & $3.600 E+01$ & $1.660 \mathrm{E}+09$ & 13.88 \\
\hline $\pm .000 \mathrm{E}-09$ & $5.527 E+17$ & 27.11 & $4.000 E+01$ & 1. $444 \mathrm{E}+09$ & 14.49 \\
\hline $\pm .000 \mathrm{E}-08$ & $4.496 E+17$ & 24.77 & $4.400 E+01$ & $1.261 \mathrm{E}+09$ & 15.20 \\
\hline $2.300 \mathrm{E}-08$ & $3.625 \mathrm{E}+17$ & 23.03 & $4.800 E+01$ & $1.104 \mathrm{E}+09$ & 16.01 \\
\hline $5.000 \mathrm{E}-08$ & $2.513 E+17$ & 21.35 & $5.200 E+01$ & $9.690 E+08$ & 16.84 \\
\hline $1.150 \mathrm{E}-07$ & $1.831 E+17$ & 19.44 & $5.600 E+01$ & $8.228 E+08$ & 17.67 \\
\hline $2.550 E-07$ & 2. $270 E+17$ & 17.51 & $5.000 E+01$ & $7.107 E+08$ & 18.49 \\
\hline $5.500 E-07$ & $6.461 \mathrm{E}+16$ & 15.87 & $6.400 \mathrm{E}+01$ & $6.147 E+08$ & 19.29 \\
\hline ¿.275E-06 & $3.621 E+16$ & 14.54 & $5.800 \mathrm{E}+01$ & $5.143 E+08$ & 20.04 \\
\hline $2.800 \mathrm{E}-06$ & $1.317 E+16$ & 13.50 & $7.200 E+01$ & $4.542 E+08$ & 20.75 \\
\hline $6.300 E-06$ & $8.538 E+15$ & 12.73 & $7.600 E+01$ & $4.019 E+08$ & 21.40 \\
\hline $1.350 \mathrm{E}-05$ & $4.185 E+15$ & 12.17 & $8.000 E+01$ & $3.508 E+08$ & 21.99 \\
\hline $3.000 E-05$ & $-.692 E+15$ & 11.75 & $8.400 E+01$ & $3.128 \mathrm{E}+08$ & 22.49 \\
\hline $5.900 E-05$ & $1.030 E+15$ & 11.59 & $8.800 E+01$ & $2.802 E+08$ & 22.92 \\
\hline $1.000 E-04$ & $5.906 \mathrm{E}+14$ & 11.43 & $9.200 E+0 I$ & $2.524 E+08$ & 23.26 \\
\hline $2.800 E-04$ & $3.701 E+14$ & $: 1.13$ & $9.600 E+91$ & $2.328 E+08$ & 23.51 \\
\hline $5.750 E-04$ & $1.754 E+14$ & 10.87 & $1.000 E+.02$ & $2.065 E+08$ & 23.69 \\
\hline $1.275 E-03$ & $1.190 \mathrm{E}+14$ & 20.58 & I. $100 E+02$ & $1.802 E+08$ & 23.80 \\
\hline $2.000 E-03$ & $8.694 E+13$ & 10.29 & $1.200 E+\hat{j} 2$ & $1.622 E+08$ & 23.81 \\
\hline $3.400 \mathrm{E}-03$ & $7.062 \mathrm{E}+13$ & 9.96 & $1.300 \mathrm{E}+02$ & $1.506 \mathrm{E}+08$ & 23.74 \\
\hline $5.500 \mathrm{E}-03$ & $4.917 E+13$ & 9.61 & $1.400 E+\hat{0} 2$ & $1.344 E+08$ & 23.59 \\
\hline $9.200 E-03$ & $4.586 \mathrm{E}+13$ & 9.31 & I. $500 \mathrm{E} \div 02$ & $1.277 E+08$ & 23.39 \\
\hline 1.200E-02 & $1.848 E+13$ & 8.26 & $1.600 \mathrm{E}+02$ & $1.227 \mathrm{E}+08$ & 23.17 \\
\hline $5.250 \mathrm{E}-02$ & I. $187 E+13$ & 7.00 & $1.700 \mathrm{E}+02$ & $1.150 E+08$ & 22.96 \\
\hline $1.000 E-01$ & $7.972 E+12$ & 6.39 & $1.800 E+02$ & $1.163 E+08$ & 22.78 \\
\hline $2.000 E-01$ & $4.529 E+12$ & 5.83 & $1.900 E+02$ & $1.088 E+08$ & 22.67 \\
\hline $5.000 E-01$ & $1.865 E+12$ & 5.56 & $2.000 E+02$ & $1.033 \mathrm{E}+08$ & 22.75 \\
\hline $1.000 \mathrm{E}+00$ & $7.760 E+11$ & 7.26 & $2.200 E+02$ & $9.676 E+07$ & 23.03 \\
\hline $2.000 E+00$ & $3.697 \mathrm{E}+11$ & 10.68 & $2.400 E \div 02$ & $9.308 E+07$ & 23.49 \\
\hline $3.000 E+00$ & $2.077 E+11$ & 13.78 & $2.600 E+02$ & $8.721 E+07$ & 24.13 \\
\hline $4.000 \mathrm{E}+00$ & $1.182 E+11$ & 15.42 & $2.800 E+32$ & $8.796 E+07$ & 24.98 \\
\hline $5.000 \mathrm{E}+00$ & $6.908 E+10$ & 15.66 & $3.000 E-02$ & $8.618 E+07$ & 26.04 \\
\hline $6.000 E+00$ & $3.717 E+10$ & 15.73 & $3.200 E+02$ & $8.612 E+07$ & 27.30 \\
\hline $7.000 E+00$ & $2.378 \mathrm{E}+10$ & 15.67 & $3.400 E+02$ & $8.622 E+07$ & 28.75 \\
\hline $8.000 E+00$ & $1.821 E+10$ & 15.50 & $3.600 E+02$ & $8.348 E+07$ & 30.35 \\
\hline $9.000 E+00$ & $1.460 \mathrm{E}+10$ & 15.24 & $3.800 E+02$ & $8.083 E+07$ & 32.10 \\
\hline $1.000 E+01$ & $1.157 \mathrm{E}+10$ & 14.93 & $4.000 E+02$ & $7.896 \mathrm{E}+07$ & 32.99 \\
\hline $1.100 E+01$ & $9.780 E+09$ & 14.57 & $4.200 E+02$ & $7.498 \mathrm{E}+07$ & 32.86 \\
\hline $1.200 E+01$ & $7.930 E+09$ & 14.17 & $4.400 E+02$ & $7.090 \mathrm{E}+07$ & 32.60 \\
\hline $1.300 E+01$ & $6.482 \mathrm{E}+09$ & 13.75 & $4.600 E+32$ & $6.797 \mathrm{E}+07$ & 32.17 \\
\hline $1.400 E+01$ & $5.6 i 4 E+09$ & 13.34 & $4.800 E+02$ & $6.384 \mathrm{E}+07$ & 31.56 \\
\hline $1.500 E+01$ & $4.884 E+09$ & 12.94 & $5.000 E+02$ & $6.081 E+07$ & 30.75 \\
\hline $1.600 E+01$ & $4.262 \mathrm{E}+09$ & 12.59 & $5.200 E+02$ & $5.680 \mathrm{E}+07$ & 29.74 \\
\hline $1.700 \mathrm{E}+01$ & $3.878 \mathrm{E}+09$ & 12.31 & $5.400 E+02$ & $5.387 E+07$ & 28.53 \\
\hline $1.800 E+01$ & $3.680 \mathrm{E}+09$ & 12.11 & $5.600 E+J 2$ & $4.877 E+07$ & 25.95 \\
\hline $1.900 E+01$ & $3.505 E+09$ & 12.00 & $6.000 E+02$ & $4.229 E+07$ & 21.91 \\
\hline $2.000 E+01$ & $3.110 E+09$ & 12.13 & $6.400 E+02$ & $3.804 E+07$ & 17.58 \\
\hline $2.400 E+01$ & $2.561 E+09$ & 12.46 & $6.800 E+02$ & $3.375 E+07$ & 13.00 \\
\hline $2.800 E+01$ & $2.240 E+09$ & 12.86 & $7.200 E+02$ & $3.057 E+07$ & 8.28 \\
\hline $3.200 E+01$ & $1.920 E+09$ & 13.34 & $7.600 E+.92$ & $2.780 E+07$ & 3.93 \\
\hline
\end{tabular}




\begin{tabular}{|c|c|c|c|}
\hline ENERGY & ELUX & ELUENCE & SDEV \\
\hline DTAL & $7.730 \mathrm{E}+12$ & $1.505 E+19$ & 7.08 \\
\hline $\begin{array}{l}Z .000 E-10 \\
Z . .50 E-07 \\
1.350 E-05 \\
1.000 E-01 \\
1.000 E+00 \\
1.000 E+01 \\
2.000 E+01 \\
A .000 E+01 \\
.000 E+02 \\
2.000 E+02\end{array}$ & $\begin{array}{l}3.755 E+10 \\
2.873 E+11 \\
2.484 E+12 \\
3.088 E+12 \\
1.634 E+12 \\
5.158 E+10 \\
4.596 E+10 \\
3.890 E+10 \\
1.424 E+10 \\
3.355 E+10\end{array}$ & $\begin{array}{l}7.310 E+16 \\
5.592 E+17 \\
4.835 E+18 \\
5.012 E+18 \\
3.182 E+18 \\
1.199 E+17 \\
3.947 E+16 \\
7.573 E+16 \\
2.773 E+16 \\
6.531 E+16\end{array}$ & 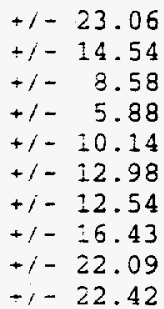 \\
\hline
\end{tabular}

SUMMARY OF INTEGRATED GROUP ENERGY FLUXES AND FLUENCES

\begin{tabular}{|c|c|c|c|c|c|}
\hline $\begin{array}{l}\text { ENERGY } \\
\text { MeV }\end{array}$ & $\begin{array}{c}F=U X \\
n / c m 2-s-m A\end{array}$ & $\begin{array}{l}\text { ELUX } \\
\mathrm{n} / \mathrm{cm} 2-\mathrm{s}\end{array}$ & $\begin{array}{l}\text { FLUENCE } \\
\mathrm{n} / \mathrm{cm} 2\end{array}$ & $\begin{array}{r}\text { STANDARD } \\
\text { EEVIATION }\end{array}$ & 8 \\
\hline$<1.0$ & $2.809 E+12$ & 8. $530 E+11$ & $5.468 \mathrm{E}+18$ & +-8.58 & \\
\hline $0.1-1.0$ & $3.088 \mathrm{E}+12$ & $9.488 E+11$ & $12 \mathrm{E}+18$ & $-1-5.88$ & \\
\hline $1.0-10.0$ & $=.534 E+12$ & $5.021 E+11$ & $3.182 E+18$ & $-1-10.14$ & \\
\hline $10.0-20.0$ & $5.258 E+10$ & $1.892 \mathrm{E}+10$ & $1.199 E+17$ & $+1-12.98$ & \\
\hline $20.0-40.0$ & $4.596 E+10$ & 1. $412 E+10$ & $7 E+16$ & $\rightarrow-1$ & \\
\hline $40.0-100.0$ & $3.890 E+10$ & $1.195 \mathrm{E}+10$ & $7.573 E+16$ & $+/-1$ & \\
\hline $10.0-200.0$ & $1.424 \mathrm{E}+10$ & $4.376 E+09$ & $2.773 \Xi+16$ & --22.09 & \\
\hline$>200.0$ & $3.355 E+10$ & $1.031 \mathrm{E}+10$ & $6.531 E+16$ & $-1-22.42$ & \\
\hline TOTAL & $7.730 E+12$ & $2.375 E+12$ & 1. $505 E+19$ & -7.08 & \\
\hline
\end{tabular}

THE PERCENTAge OF NEUTRONS ABOVE $20 \mathrm{MeV}$ is -1.72

RELATIVE COVARIANCES $(10 \times 10)$

$\begin{array}{rrrrrrrrrr}1000 & 820 & -95 & -240 & -236 & -47 & 104 & 50 & 12 & 5 \\ 1000 & 354 & 63 & -9 & -26 & 59 & 71 & 2 \vdots & 15 & \\ 1000 & 598 & 798 & 277 & -134 & -111 & 55 & 5 \vdots & \\ 1000 & 941 & 452 & -152 & -194 & 55 & 89 & & \\ 1000 & 545 & -112 & -260 & 51 & 117 & & & \\ 1000 & 463 & -145 & -121 & 124 & & & & \\ 1000 & 598 & -224 & -207 & & & & & \\ 1000 & 288 & -563 & & & & & & \\ 1000 & -184 & & & & & & & \\ 1000 & & & & & & & \end{array}$


- FRACTIONAL ERRORS IN INPUT SPECTRUM $.0300 \quad .0300 \quad 3.2550$

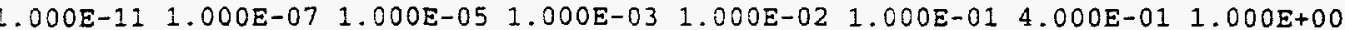

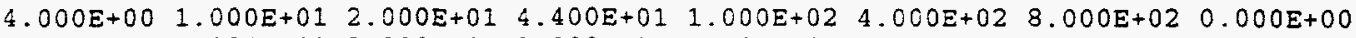

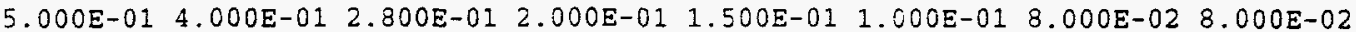
$2.500 E-013.000 E-013.500 E-01 \quad 6.000 E-01 \quad 5.000 E-01 \quad 6.000 E-01 \quad 0.000 E+00 \quad 0.000 E+00$

$7.504 E+17 \quad 4.184 E+17 \quad 3.101 E+17 \quad 2.602 E+17 \quad 1.928 E+17 \quad 1.439 E+17 \quad 8.561 E+16 \quad 5.704 E+16$ $\begin{array}{llllllll}3.481 E+16 & 2.008 E+16 & 1.256 E+16 & 6.608 E+15 & 3.191 E+15 & 2.259 E+15 & 9.518 E+14 & 4.433 E+14\end{array}$ $2.262 \mathrm{E}+14 \quad 1.651 \mathrm{E}+14 \quad 1.251 \mathrm{E}+14 \quad 9.515 \mathrm{E}+13 \quad 7.253 \mathrm{E}+13 \quad 6.685 \mathrm{E}+13 \quad 3.442 \mathrm{E}+13 \quad 2.139 \mathrm{E}+13$

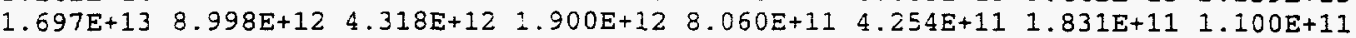
$6.854 \mathrm{E}+10 \quad 3.759 \mathrm{E}+10 \quad 2.770 \mathrm{E}+10 \quad 2.111 \mathrm{E}+10 \quad 1.544 \mathrm{E}+10 \quad 1.345 \mathrm{E}+10 \quad 1.159 \mathrm{E}+10 \quad 1.001 \mathrm{E}+10$ $9.445 E+09 \quad 9.124 E+09 \quad 8.827 E+09 \quad 8.552 E+09 \quad 8.339 E+09 \quad 8.155 E+09 \quad 7.293 E+09 \quad 6.237 E+09$

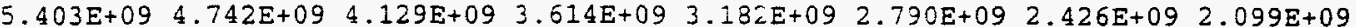

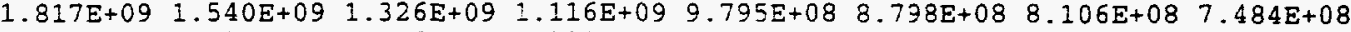
$\begin{array}{llllllll}6.983 E+08 & 6.527 E+08 & 5.937 E+08 & 5.329 E+08 & 4.860 E+08 & 4.336 E+08 & 4.083 E+08 & 3.820 E+08\end{array}$ $\begin{array}{llllllll}3.588 E+08 & 3.383 E+08 & 3.284 E+08 & 3.225 E+08 & 3.235 E+08 & 3.295 E+08 & 3.257 E+08 & 3.286 E+08\end{array}$ $\begin{array}{lllllllll}3.508 E+08 & 3.544 E+08 & 3.477 E+08 & 3.278 E+08 & 3.101 E+08 & 2.993 E+08 & 2.896 E+08 & 2.668 E+08\end{array}$

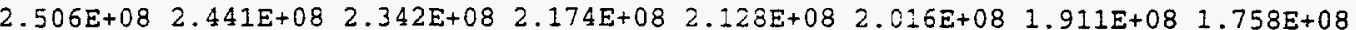
1. $623 \mathrm{E}+08 \quad 1.479 \mathrm{E}+08 \quad 1.375 \mathrm{E}+08 \quad 1.260 \mathrm{E}+08$

\begin{tabular}{|c|c|c|c|c|c|}
\hline $\begin{array}{c}100 \quad \text { NFOIL }= \\
\text { REACTIONS }\end{array}$ & $\begin{array}{l}13 \\
\text { COVER }\end{array}$ & $\begin{array}{r}\text { ACTIVITY NORM= } \\
\text { ACTIVITIES }\end{array}$ & \multicolumn{3}{|c|}{$3.255 E+00$} \\
\hline FE54 (N, *)MN54 & .00 & $8.251 \mathrm{E}-13$ & & & .00 \\
\hline FE58(N,G)FE59 & .00 & $3.299 \mathrm{E}-13$ & IFX & FENG & 4.76 \\
\hline $\cos 9(\mathrm{~N}, 2 \mathrm{~N}) \cos 8$ & .00 & 1. $099 \mathrm{E}-13$ & & & .00 \\
\hline $\cos 9(\mathrm{~N}, 3 \mathrm{~N}) \cos 7$ & .00 & $3.542 \mathrm{E}-14$ & & & .00 \\
\hline $\operatorname{Co5} 9(N, G) \operatorname{CO} 50$ & .00 & $9.501 E-12$ & IFX & CONG & 1.96 \\
\hline $\mathrm{NI} 60(\mathrm{~N}, \mathrm{P}) \mathrm{CO} 60$ & .00 & $2.959 E-14$ & & & .00 \\
\hline $\mathrm{CU}(\mathrm{N}, \bullet) 54 \mathrm{MN}$ & .00 & $3.913 E-15$ & & & .00 \\
\hline $\mathrm{CU}\left(\mathrm{N},{ }^{*}\right) 59 \mathrm{FE}$ & .00 & $2.057 \mathrm{E}-15$ & & & .00 \\
\hline$C U(N, *) 56 C O$ & .00 & $2.027 E-15$ & & & .00 \\
\hline$C U(N, *) 57 C 0$ & .00 & $9.749 E-15$ & & & .00 \\
\hline$C U(N, *) 58 C 0$ & .00 & $2.600 \mathrm{E}-14$ & & & .00 \\
\hline $\mathrm{CU}(\mathrm{N}, *) 60 \mathrm{CO}$ & .00 & $\therefore .037 \mathrm{E}-14$ & & & .00 \\
\hline $\operatorname{NB} 93\left(\mathrm{~N}, \mathrm{~N}^{\prime}\right) \mathrm{NB} 93 \mathrm{M}$ & .00 & $8.574 \mathrm{E}-13$ & & & .00 \\
\hline
\end{tabular}

COVARIANCE PARAMETERS

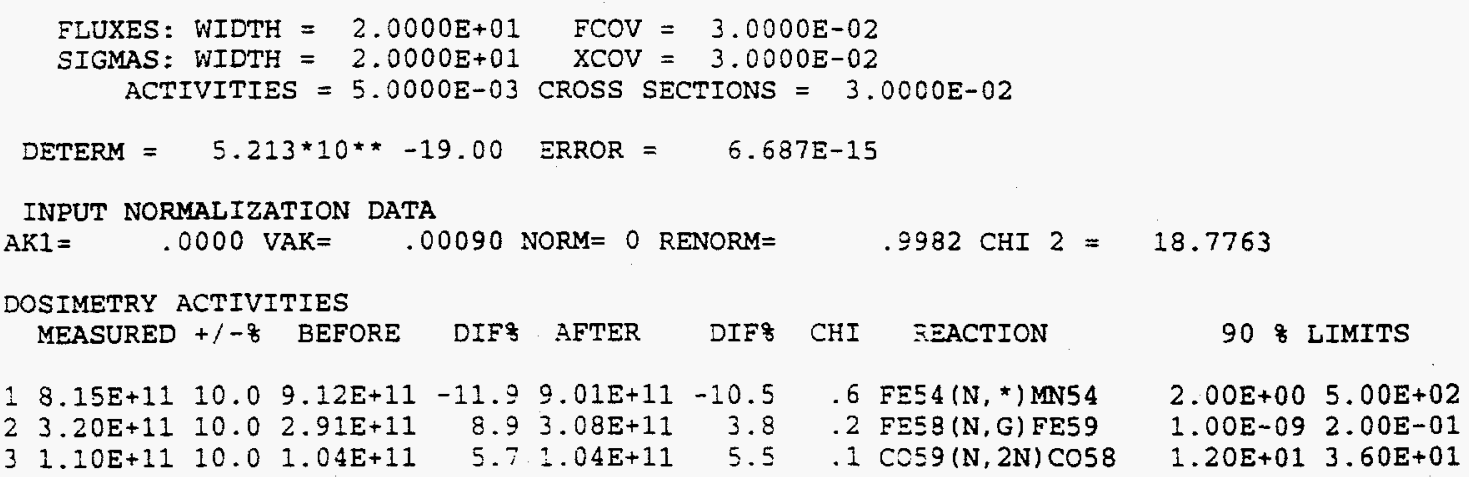


$43.64 \mathrm{E}+10 \quad 10.02 .93 \mathrm{E}+10$

$59.50 \mathrm{E}+1215.0$ 9. $32 \mathrm{E}+12$

$62.96 \mathrm{E}+10 \quad 15.0 \quad 3.12 \mathrm{E}+10$

$73.91 \mathrm{E}+0910.03 .44 \mathrm{E}+09$

$1.06 E+0925.02 .71 E+08$

92.03E+09 $10.02 .37 E+09$

$109.75 E+0910.01 .09 E+10$

$11.60 \mathrm{E}+10 \quad 10.01 .61 \mathrm{E}+10$

$121.04 E+1025.03 .16 E+09$

$138.57 E+1120.08 .55 E+11$

STD. DEV.

DOSIMETRY DATA INPUT CORRELATION MATRIX

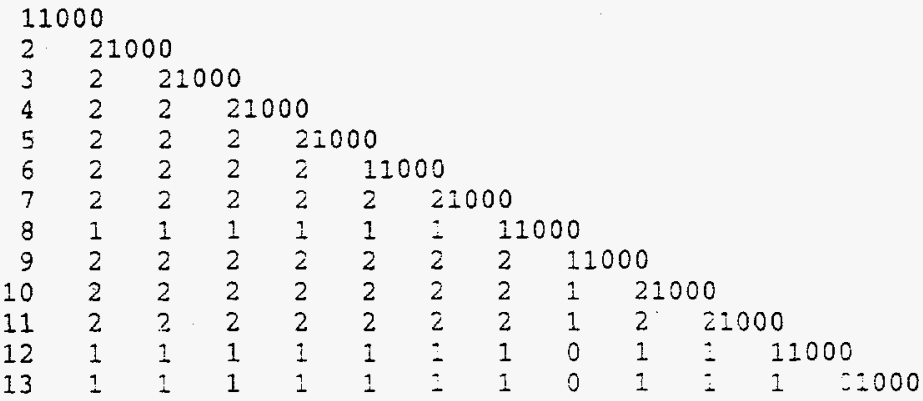

RELATIVE COV. MATRIX OF ACTIVITIES

$$
\text { O CORREIATION MATRIX }
$$

125.931000

$222.82 \quad 2241000$

$3 \quad 35.81788 \quad 1341000$

$452.24601 \quad 556491000$

$\begin{array}{llllll}5 & 35.00 & 140 & 693 & 73 & 281000\end{array}$

$\begin{array}{lllllll}6 & 30.58 & 726 & 278 & 783 & 489 & 1701000\end{array}$

$\begin{array}{llllllll}7 & 44.82 & 405 & 21 & 114 & 137 & \$ 2 & 1081000\end{array}$

$\begin{array}{lllllllll}8 & 42.28 & 460 & 23 & 169 & 190 & =3 & 142 & 7881000\end{array}$

$\begin{array}{llllllllll}9 & 42.57 & 508 & 23 & 206 & 234 & \vdots 3 & 161 & 794 & 8151000\end{array}$

$\begin{array}{lllllllllll}10 & 36.77 & 688 & 36 & 448 & 437 & 19 & 322 & 712 & 758 & 8121000\end{array}$

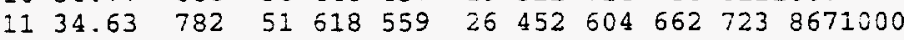

$\begin{array}{lllllllllllll}12 & 35.54 & 707 & 41 & 486 & 459 & 21 & 355 & 698 & 744 & 795 & 879 & 8701000\end{array}$

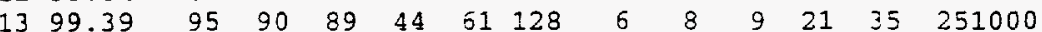

CONTRIBUTION DUE TO INPUT ELUX COV. MATRIX

के CORRELATION. MATRIX

123.961000

$2 \quad 21.48 \quad 2411000$

$3 \quad 33.83 \quad 892 \quad 1391000$

$437.75 \quad 890 \quad 70 \quad 9441000$

$\begin{array}{llllll}5 & 25.46 & 194 & 996 & 95 & 431000\end{array}$

$\begin{array}{lllllll}6 & 27.91 & 848 & 309 & 899 & 733 & 2431000\end{array}$

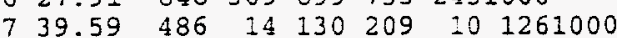

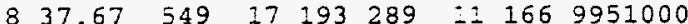

$939.04 \quad 590 \quad 16 \quad 231.347 \quad \leq 0 \quad 184 \quad 974 \quad 9911000$

$\begin{array}{lllllllllll}10 & 34.48 & 783 & 29 & 498 & 639 & \div 8 & 367 & 853 & 901 & 9381000\end{array}$

$\begin{array}{llllllllllll}11 & 32.78 & 883 & 44 & 683 & 810 & 27 & 513 & 715 & 778 & 826 & 9681000\end{array}$

$\begin{array}{lllllllllllll}12 & 33.11 & 810 & 34 & 544 & 675 & 21 & 408 & 841 & 889 & 924 & 998 & 9781000\end{array}$

$1316.04 \quad 611 \quad 568 \quad 566 \quad 361 \quad 495 \quad 848 \quad 28 \quad 42 \quad 44 \quad 125 \quad 209 \quad 1511000$

CONTRIBUTION DUE TO INPUT X-SEC. COV. MATRIX

CORRELATION MATRIX

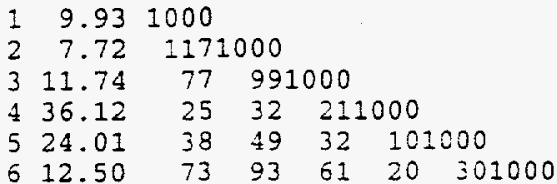




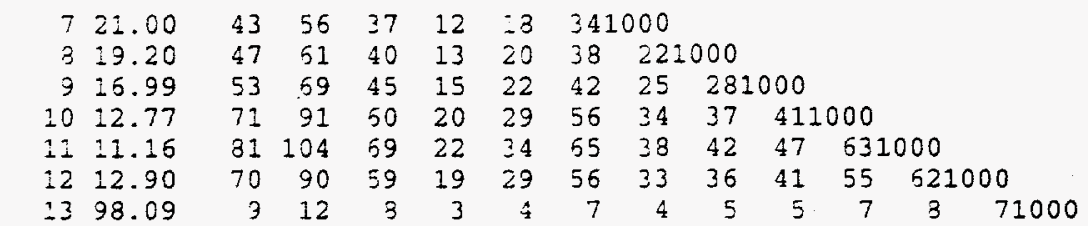

DIFFERENTIAL FLUXES INPUT NORMALIZED BY
$G$ ENERGY NEW

.9982

$\therefore .000 \mathrm{E}-10 \quad 8.631 \mathrm{E}+17 \quad 7.490 \mathrm{E}+17$

I. $000 E-09 \quad 4.793 E+17 \quad 4.175 E+17$

$1.000 \mathrm{E}-08$
$2.300 \mathrm{~B}-08$

$2.300 \mathrm{E}-08 \quad 2.970 \mathrm{E}+17 \quad 2.597 \mathrm{E}+17$

$\begin{array}{lll}5.000 E-08 & 2.195 \mathrm{E}+17 & 1.925 \mathrm{E}+17\end{array}$

$1.150 \mathrm{E}-07 \quad 1.629 \mathrm{E}+17 \quad 1.436 \mathrm{E}+17$

$2.550 \mathrm{E}-07 \quad 9.516 \mathrm{E}+16 \quad 8.546 \mathrm{E}+16$

5. $500 E-07$

1. $275 E-06$

$2.800 E-06$

$5.300 E-06$

1.350E-05

$3.000 E-05$

$6.900 \mathrm{E}-05$

$1.000 \mathrm{E}-04$

$2.800 \mathrm{E}-04$

$5.750 \mathrm{E}-04$

5. $353 \mathrm{E}+16$

$5.694 \mathrm{E}+16$

$3.843 E+16 \quad 3.475 E+16$

$2.197 E+16 \quad 2.004 E+16$

$1.362 E+16 \quad 1.254 E+16$

$7.107 \bar{E}+15 \quad 6.596 \mathrm{E}+15$

$3.403 E+15$

3. $185 E+15$

$2.285 E+15 \quad 2.155 E+15$

$9.997 E+14$

$9.501 E+14$

$4.619 E+14 \quad 4.425 E+14$

$2.339 \mathrm{E}+14 \quad 2.258 \mathrm{E}+14$

$1.696 \mathrm{E}+14 \quad 1.648 \mathrm{E}+14$

2. $000 \mathrm{E}-03$

1. $277 \mathrm{E}+14$

1. $249 \mathrm{E}+14$

3. $400 \mathrm{E}-03$

9. $655 E+13$

$9.498 \mathrm{E}+13$

$5.500 E-03$

$7.323 E+13$

$7.240 \mathrm{E}+13$

$9.200 E-03$

6. $720 E+13$

$6.573 E+13$

1. 200E-02

3. $446 \mathrm{E}+13$

$3.436 \mathrm{E}+13$

$1.000 \mathrm{E}-01$

2. $136 \mathrm{E}+13$

$2.135 E+13$

2.000E-01

1. $691 \mathrm{E}+13$

$1.694 \mathrm{E}+13$

$5.000 \mathrm{E}-01$

$8.955 E+12$

$8.982 E+12$

$1.000 E+00$

$4.293 E+12$

$4.310 E+12$

$1.883 \mathrm{E}+12 \quad 1.897 \mathrm{E}+12$

2. $3000 E+00$

$7.938 E+11$

$8.045 E+11$

$4.164 E+11$

$4.246 \mathrm{E}+11$

$4.000 \mathrm{E}+00$

$1.785 \mathrm{E}+11$

1. $828 \mathrm{E}+11$

$5.000 E+00$

$6.071 \mathrm{E}+11$

$1.098 E+11$

$7.000 E+00$

3. $562 \mathrm{E}+10$

$6.842 E+10$

8. $000 E+00$

$2.702 \mathrm{E}+10$

$2.752 E+10$

$B Y$

STD DEV *

NEW

OLD

RATIO

INT FLUX >+-

1.148

1.145

33.49

46.08

.727

1.14326 .39

1. $141 \quad 24.68$

1. 134

1.125

1.116

22.60

20.40

18.45

$1.106 \quad 16.77$

$1.096 \quad 15.36$

1.087

1.077

I. 068

1.060

1.052

14.22

13.32

12.58

43.59

41.96

.698

1. $508 \mathrm{E}+13$

41.15

40.22

38.49

35.24

34.06

32.00

30.10

28.35

28.35
26.77

25.25

12.16

1.044

1.036

11.76

24.17

22.99

11.28

1.029

1.022

1.017

10.87

21.59

20.33

10.48

10.12

19.10

19.10
18.00

9.73

16.92

.011
1.007

1.003

1.000

.998

.997

.996

.993

.987

.981

.977

.976

.975

976

.977

.979

.982

$1.000 \mathrm{E}+01$

2. $064 \mathrm{E}+10$

2. $107 E+10$

$1.100 \mathrm{E}+01$

1. $323 \bar{E}+10$

$1.541 \mathrm{E}+10$

$1.200 \mathrm{E}+01$

1. $144 \mathrm{E}+10$

1. $343 E+10$

1. $300 \mathrm{E}+01$

$9.916 E+09$

1. $157 \mathrm{E}+10$

$9.992 E+09$

i. $400 \mathrm{E}+01$

$9.391 E+09$

$9.428 \mathrm{E}+09$

1. $500 \mathrm{E}+01$

$9.104 \mathrm{E}+09$

9.108E+09

9.35

9.03

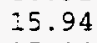

7.99

6.76

6.16

5.61

5.34

6.93

10.14

13.04

14.53

14.69

14.70

14.59

14.38

14.10

13.77

5.14

$\square 3.20$

11.01

9.93

8.98

8.54

$\because 1.47$

17.19

22.56

25.73

26.77

27.67

28.47

29.18

29.83

30.46

13.43

989

13.07

31.07

31.65

12.71

32.19

.996

1.000

12.37

32.70

12.07

33.19

1.003

$1.700 \mathrm{E}+01 \quad 8.583 \mathrm{E}+09$

$8.811 E+09$

$8.537 E+09$

1.005

11.84

33.65

1.007

$\begin{array}{ll}8.324 E+09 & 1.007 \\ 8.140 E+09 & 1.009\end{array}$

7. $280 \mathrm{E}+09$

11.68

34.09

.641

1. $508 \mathrm{E}+13$

6.69

6.70

6.70

6.70

1. $507 E+13$

6.71

6.71

$587 \quad 1.505 E+13$

$\begin{array}{ll}563 & 1.503 E+13\end{array}$

6.72

6.73

$.524 \quad 1.495 E+13$

6.75

6.76

6.78

6.79

6.80

$498 \quad 1.42 E+13$

$.503 \quad 1.447 \mathrm{E}+13$

6.81

$1.440 \mathrm{E}+13$

6.81

$.5221 .422 \mathrm{E}+13$

6.80

6.79

6.77

$5491.392 \mathrm{E}+13$

$.5621 .380 \mathrm{E}+13$

6.75

6.73

1. $362 \mathrm{E}+13$

6.70

6.67

$596 \quad 1.314 \mathrm{E}+13$

6.64

6.52

6.52

6.62

7.12

8.20

9.51

9.39

8.25

7.37

6.90

6.75

6.76

6.83

6.92

7.00

.452

.432

.395

.378

.364

.352
.343

11.65 


\begin{tabular}{|c|c|c|c|c|c|c|c|c|c|}
\hline 54 & $3.800 E+01$ & $6.755 \mathrm{E}+08$ & $7.470 E+08$ & .904 & 25.62 & 48.66 & .527 & $1.913 \mathrm{E}+11$ & 13.71 \\
\hline 65 & $9.200 \mathrm{E}+01$ & $6.270 E+08$ & $6.970 E+08$ & .899 & 26.06 & 49.23 & .529 & $1.886 E+11$ & 13.99 \\
\hline 56 & $9.600 E+01$ & $5.837 E+08$ & $5.515 E+08$ & .896 & 26.39 & 49.79 & .530 & $1.861 E+11$ & 14.25 \\
\hline 67 & 1. $000 E+02$ & $5.297 E+08$ & $5.926 E+08$ & .894 & 26.61 & 50.38 & .528 & $1.838 \mathrm{E}+11$ & 14.49 \\
\hline 68 & $1.100 E+02$ & $4.750 E+08$ & $5.319 E+08$ & .893 & 26.72 & 50.99 & .524 & $1.785 \mathrm{E}+11$ & 15.06 \\
\hline 69 & $1.200 E+02$ & $4.336 E+08$ & $4.851 E+08$ & .894 & 25.69 & 51.55 & .518 & $1.737 \mathrm{E}+11$ & 15.60 \\
\hline 70 & $1.300 \mathrm{E}+02$ & $3.923 E+08$ & $4.378 E+08$ & .896 & 26.53 & 52.07 & .510 & $1.694 \mathrm{E}+11$ & 16.11 \\
\hline 71 & $2.400 E+02$ & $3.658 E+08$ & $4.076 E+08$ & .900 & 26.25 & 52.56 & .499 & $1.655 \mathrm{E}+11$ & 16.57 \\
\hline 72 & $1.500 E+02$ & $3.452 E+08$ & $3.813 E+08$ & .905 & 25.87 & 53.02 & .488 & 1. $618 \mathrm{E}+11$ & 17.01 \\
\hline 73 & $1.600 E+02$ & $3.267 E+08$ & $3.582 E+08$ & .912 & 25.41 & 53.46 & .475 & 1. $583 \mathrm{E}+11$ & 17.42 \\
\hline 74 & $1.700 E+02$ & $3.108 \mathrm{E}+08$ & $3.377 \mathrm{E}+08$ & 920 & 24.90 & 53.88 & .462 & $1.551 E+11$ & 17.79 \\
\hline 75 & $1.800 E+02$ & $3.047 \varepsilon+08$ & $3.278 E+08$ & .929 & 4.36 & 54.27 & .449 & $1.520 E+11$ & 18.13 \\
\hline 76 & $1.900 E+02$ & $3.025 E+08$ & $3.219 E+08$ & .940 & 23.85 & 54.65 & .436 & 1. $489 \mathrm{E}+11$ & 18.44 \\
\hline 77 & $2.000 E+02$ & $3.069 E+08$ & $3.229 E+08$ & 950 & .48 & 55.17 & .425 & $1.459 \mathrm{E}+11$ & .72 \\
\hline 78 & $2.200 \mathrm{E}+02$ & $3.069 E+08$ & $3.190 \mathrm{E}+08$ & .962 & 23.27 & 55.84 & .417 & 1. $398 \mathrm{E}+11$ & 19.26 \\
\hline 79 & $2.400 E+02$ & $3.166 E+08$ & $3.251 E+08$ & .974 & 23.21 & 56.45 & .411 & $1.336 \mathrm{E}+11$ & 19.77 \\
\hline 80 & $2.600 E+02$ & $3.235 E+08$ & $3.280 E+08$ & .986 & 23.35 & 57.03 & .409 & $1.273 E+11$ & 20.27 \\
\hline 81 & $2.800 E+02$ & $3.498 E+08$ & $3.502 \mathrm{E}+08$ & .999 & 23.72 & 57.56 & .412 & $1.208 \mathrm{E}+11$ & 20.73 \\
\hline 82 & $3.000 E+02$ & $3.579 E+08$ & $3.538 E+08$ & 1.012 & .34 & 58.07 & .419 & $1.138 \mathrm{E}+11$ & 21.17 \\
\hline 83 & $3.200 E+02$ & $3.555 E+08$ & $3.471 E+08$ & 1.024 & .24 & 58.55 & .431 & $1.067 \mathrm{E}+11$ & 21.53 \\
\hline 84 & $3.400 E+02$ & $3.391 E+08$ & $3.272 E+08$ & 1.036 & 5.40 & 59.00 & .447 & $9.955 E+10$ & 21.80 \\
\hline 85 & 3. $600 \mathrm{E}+02$ & $3.244 E+08$ & $3.095 E+08$ & 1.048 & .75 & 59.43 & .468 & $9.277 E+10$ & 21.93 \\
\hline 86 & $3.800 E+02$ & $3.164 E+08$ & $2.988 E+08$ & 1.059 & .39 & 59.85 & .491 & 8. $628 E+10$ & 21.89 \\
\hline 87 & $4.000 E+02$ & $3.085 E+08$ & $2.891 E+08$ & 1.067 & .28 & 58.55 & .517 & $7.996 E+10$ & 21.66 \\
\hline 88 & $4.200 E+02$ & $2.855 E+08$ & $2.663 E+08$ & 1.072 & .29 & 55.56 & .545 & $7.379 E+10$ & .26 \\
\hline 89 & $4.400 E+02$ & $2.689 E+08$ & $2.501 E+08$ & 1.075 & .21 & 52.56 & .575 & $6.808 E+10$ & 20.76 \\
\hline 90 & $4.600 E+02$ & $2.622 \mathrm{E}+08$ & $2.437 E+08$ & 1.076 & .98 & 49.57 & .605 & $6.270 E+10$ & 20.16 \\
\hline 91 & $4.800 E+02$ & $2.516 \bar{E}+08$ & $2.338 E+08$ & 1.076 & 29.59 & 46.58 & .635 & $5.745 E+10$ & 19.43 \\
\hline 92 & $5.000 E+02$ & $2.332 E+08$ & $2.170 E+08$ & I. 074 & 3.00 & 43.58 & .665 & $5.242 E+10$ & 18.5 \\
\hline 93 & $5.200 \mathrm{E}+02$ & $2.276 E+08$ & $2.124 \mathbf{E}+08$ & 1.072 & 28.22 & 40.59 & .695 & $4.776 \mathrm{E}+10$ & 17.67 \\
\hline 94 & $5.400 \mathrm{E}+02$ & $2.149 E+08$ & $2.012 E+08$ & 1.068 & 27.22 & 37.60 & .724 & 4. $321 E+10$ & 16.64 \\
\hline 95 & $5.600 \mathrm{E}+02$ & $2.023 E+08$ & $1.908 E+08$ & 1.061 & 24.88 & 33.12 & .751 & $3.891 E+10$ & .52 \\
\hline 96 & $6.000 E+02$ & $1.843 E+08$ & $1.755 E+08$ & 1.050 & 21.11 & 27.15 & .777 & $3.082 E+10$ & 13.14 \\
\hline 97 & $6.400 E+02$ & $1.683 E+08$ & $1.620 E+08$ & 1.039 & 17.01 & 21.20 & .802 & $2.345 E+10$ & 10.71 \\
\hline 98 & $6.800 E+02$ & $1.517 E+08$ & $1.476 E+08$ & 1.028 & .63 & 15.29 & .826 & $1.671 E+10$ & .27 \\
\hline 99 & $7.200 E+02$ & $1.395 E+08$ & $1.373 E+08$ & 1.017 & 8.08 & 9.48 & .853 & $1.064 E+10$ & .92 \\
\hline 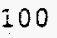 & $7.600 E+02$ & $1.265 E+08$ & 1. $258 \mathrm{E}+08$ & 1.006 & 3.88 & 4.24 & .914 & $5.061 E+09$ & 0 \\
\hline
\end{tabular}

INTEGRALS OF SPECTRA

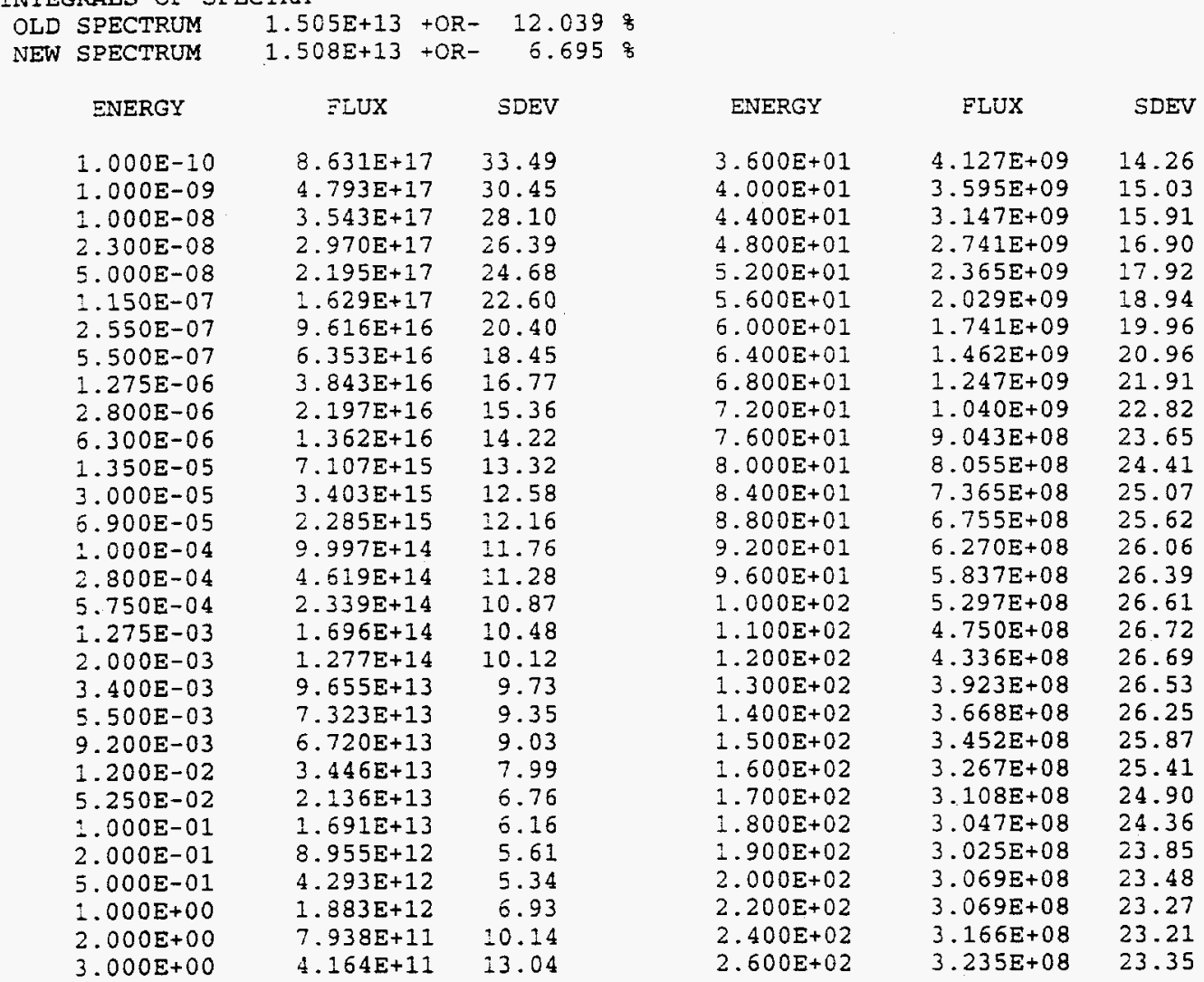




\begin{tabular}{|c|c|c|c|c|c|}
\hline$\therefore .000 \mathrm{E}+00$ & $1.735 E+11$ & 14.53 & $2.800 E+02$ & $3.498 E+08$ & 23.72 \\
\hline$\Xi .000 E+00$ & 1. $071 E+11$ & 14.69 & $3.000 E+02$ & $3.579 E+08$ & 24.34 \\
\hline$=.000 E+00$ & $5.673 E+10$ & 14.70 & $3.200 E+02$ & $3.555 E+08$ & 25.24 \\
\hline $7.000 E+00$ & $3.562 \mathrm{E}+10$ & 14.59 & $3.400 E+02$ & $3.391 E+08$ & 26.40 \\
\hline $3.000 E+00$ & $2.702 \mathrm{E}+10$ & 14.38 & $3.600 E+02$ & $3.244 E+08$ & 27.79 \\
\hline$\exists .000 E+00$ & $2.064 E+10$ & 14.10 & $3.800 E+02$ & $3.164 \mathrm{E}+08$ & 29.39 \\
\hline$\therefore .000 \mathrm{E}+01$ & $1.514 E+10$ & 13.77 & $4.000 E+02$ & $3.085 E+08$ & 30.28 \\
\hline $.100 \mathrm{E}+01$ & $1.323 E+10$ & 13.43 & $4.200 E+02$ & $2.855 E+08$ & 30.29 \\
\hline $.200 E+0 I$ & $1.144 \mathrm{E}+10$ & 13.07 & $4.400 \mathrm{E}+02$ & $2.689 E+08$ & 30.21 \\
\hline $.300 E+01$ & $9.916 E+09$ & 12.71 & $4.600 E+C 2$ & $2.622 \mathrm{E}+08$ & 29.98 \\
\hline $.400 E+01$ & $9.391 E+09$ & 12.37 & $4.800 E+02$ & $2.516 \mathrm{E}+08$ & 29.59 \\
\hline $.500 E+01$ & $9.104 E+09$ & 12.07 & $5.000 E+02$ & $2.332 E+08$ & 29.00 \\
\hline $.600 E+01$ & $8.835 E \div 09$ & 11.84 & $5.200 E+02$ & $2.276 \mathrm{E}+08$ & 28.22 \\
\hline $.700 E+01$ & $8.583 E+09$ & 11.68 & $5.400 \mathrm{E}+02$ & $2.149 \mathrm{E}+08$ & 27.22 \\
\hline $.300 \Sigma+01$ & $8.386 E+09$ & 11.61 & $5.600 E+02$ & $2.023 E+08$ & 24.88 \\
\hline $.900 E+01$ & $8.211 E+09$ & 11.65 & $6.000 E+02$ & $1.843 \mathrm{E}+08$ & 21.11 \\
\hline $.000 E+01$ & $7.347 E+09$ & 11.92 & $6.400 E+02$ & $1.683 E+08$ & 17.01 \\
\hline $.400 \mathrm{E}+01$ & $6.281 E+09$ & 12.39 & $5.800 \mathrm{E}+02$ & $1.517 E+08$ & 12.63 \\
\hline $.800 E+01$ & $5.433 E+09$ & 12.94 & $7.200 \mathrm{E}+02$ & $1.395 \mathrm{E}+08$ & 8.08 \\
\hline $.200 \mathrm{E}+01$ & $4.757 E+09$ & 13.57 & $7.600 \mathrm{E}+02$ & $1.265 E+08$ & 3.88 \\
\hline
\end{tabular}

SUMMARY OF BROAD-GROUP FLUXES, FLUENCES, AND UNCERTAINTIES

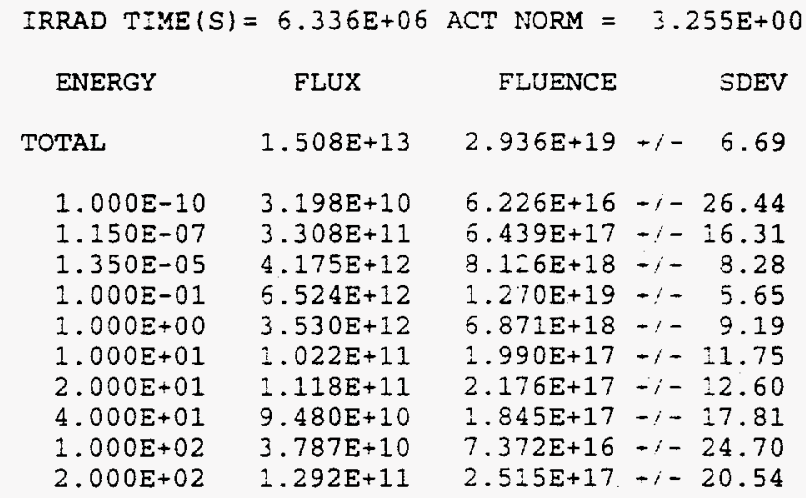

SUMMARY OF INTEGRATED GROUP ENERGY FLUXES AND FLUENCES

\begin{tabular}{|c|c|c|c|c|c|}
\hline $\begin{array}{l}\text { ĐNERGY } \\
\mathrm{MeV}\end{array}$ & $\begin{array}{c}\text { FLUX } \\
\mathrm{n} / \mathrm{cm} 2-\mathrm{s}-\mathrm{mA}\end{array}$ & $\begin{array}{l}\text { ELUX } \\
\mathrm{n} / \mathrm{cm} 2-\mathrm{s}\end{array}$ & $\begin{array}{l}\text { ELUENCE } \\
\mathrm{n} / \mathrm{Cm} 2\end{array}$ & $\begin{array}{r}\text { STANDARD } \\
\text { ¿EVIATION }\end{array}$ & 8 \\
\hline $\begin{array}{r}<1.0 \\
0.1-1.0 \\
1.0-10.0 \\
10.0-20.0 \\
20.0-40.0 \\
40.0-100.0 \\
100.0-200.0 \\
>200.0\end{array}$ & $\begin{array}{l}4.537 \mathrm{E}+12 \\
6.524 \mathrm{E}+12 \\
3.530 \mathrm{E}+12 \\
1.022 \mathrm{E}+11 \\
1.118 \mathrm{E}+11 \\
9.480 \mathrm{E}+10 \\
3.787 \mathrm{E}+10 \\
1.292 \mathrm{E}+11\end{array}$ & $\begin{array}{l}1.394 \mathrm{E}+12 \\
2.004 \mathrm{E}+12 \\
1.084 \mathrm{E}+12 \\
3.141 \mathrm{E}+10 \\
3.434 \mathrm{E}+10 \\
2.912 \mathrm{E}+10 \\
1.164 \mathrm{E}+10 \\
3.969 \mathrm{E}+10\end{array}$ & $\begin{array}{l}8.832 E+18 \\
1.270 E+19 \\
6.871 E+18 \\
1.990 E+17 \\
2.175 E+17 \\
1.845 E+17 \\
7.372 E+16 \\
2.515 E+17 \\
2.936 E+19\end{array}$ & $\begin{array}{l}-1-8.28 \\
-1-5.65 \\
-/-9.19 \\
-1-11.75 \\
-1-12.60 \\
-17.81 \\
-1-24.70 \\
-1-20.54\end{array}$ & $\begin{array}{r}30.08 \\
43.25 \\
23.40 \\
.68 \\
.74 \\
.63 \\
.25 \\
.86\end{array}$ \\
\hline
\end{tabular}

THE PERCENTAGE OF NEUTRONS ABOVE $20 \mathrm{MeV}=\Xi-2.48$

RELATIVE COVARIANCES (10X10)

$\begin{array}{rrrrrrrrrr}1000 & 849 & 3 & -194 & -209 & -25 & 97 & \vdots 3 & 9 & 10 \\ 1000 & 406 & 83 & 6 & -16 & 67 & 60 & 20 & 17 & \\ 1000 & 891 & 801 & 213 & -140 & -87 & 48 & 52 & & \\ 1000 & 947 & 370 & -175 & -166 & 43 & 75 & & & \\ 1000 & 544 & -156 & -230 & 37 & -00 & & & \\ 1000 & 502 & -87 & -152 & 82 & & & & \\ 1000 & 627 & -145 & -258 & & & & & \\ 1000 & 394 & -564 & & & & & & \\ 1000 & -223 & & & & & & & \\ 1000 & & & \end{array}$


0 FRACTIONAL ERRORS IN INPUT SPECTRUM

$161.000 E+00$

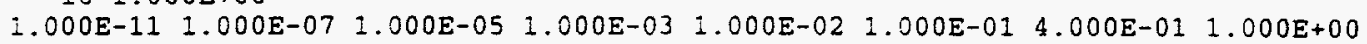
$\begin{array}{llllllll}4.000 E+00 & 1.000 E+01 & 2.000 E+01 & 4.400 E+01 & 1.000 E+02 & 4.000 E+02 & 8.000 E+02 & 0.000 E+00\end{array}$ 5.000E-01 4.000E-01 2.800E-01 2.000E-01 1.500E-01 1.000E-01 8.000E-02 8.000E-02 $2.500 E-013.000 E-013.500 E-01 \quad 4.000 E-01 \quad 5.000 E-01 \quad 6.000 E-01 \quad 0.000 E+00 \quad 0.000 E+00$ - INPUT SPECTRUM

$1001.000 \mathrm{E}+00$

$\begin{array}{llllllll}1.039 E+18 & 3.399 E+17 & 4.029 E+17 & 2.213 E+17 & 1.621 E+17 & 1.200 E+17 & 8.277 E+16 & 4.945 E+16\end{array}$ $\begin{array}{llllllll}3.329 E+16 & 2.022 E+16 & 1.027 E+16 & 6.343 E+15 & 3.420 E+15 & 2.453 E+15 & 1.208 E+15 & 5.970 E+14\end{array}$ $4.179 \mathrm{E}+14 \quad 2.371 \mathrm{E}+14 \quad 1.446 \mathrm{E}+14 \quad 1.204 \mathrm{E}+14 \quad 7.341 \mathrm{E}+13 \quad 6.990 \mathrm{E}+13 \quad 2.876 \mathrm{E}+13 \quad 1.967 \mathrm{E}+13$ $\begin{array}{llllllll}1.316 E+13 & 7.900 E+12 & 3.179 E+12 & 1.254 E+12 & 5.731 E+11 & 3.230 E+11 & 1.990 E+11 & 1.179 E+11\end{array}$ $7.748 E+10 \quad 4.889 E+10 \quad 3.494 E+10 \quad 2.624 E+10 \quad 1.823 E+10 \quad 1.551 E+10 \quad 1.329 E+10 \quad 1.235 E+10$ $1.123 \mathrm{E}+10 \quad 9.963 \mathrm{E}+09 \quad 8.848 \mathrm{E}+09 \quad 8.064 \mathrm{E}+09 \quad 7.733 \mathrm{E}+09 \quad 6.851 \mathrm{E}+09 \quad 6.063 \mathrm{E}+09 \quad 4.979 \mathrm{E}+09$ $4.164 \mathrm{E}+09 \quad 3.585 \mathrm{E}+09 \quad 3.113 \mathrm{E}+09 \quad 2.672 \mathrm{E}+09 \quad 2.305 \mathrm{E}+09 \quad 1.996 \mathrm{E}+09 \quad 1.761 \mathrm{E}+09 \quad 1.555 \mathrm{E}+09$

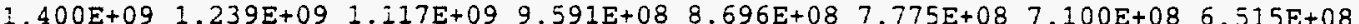
$\begin{array}{llllllll}5.911 E+08 & 5.590 \mathrm{E}+08 & 4.991 \mathrm{E}+08 & 4.467 \mathrm{E}+08 & 3.914 \mathrm{E}+08 & 3.597 \mathrm{E}+08 & 3.235 \mathrm{E}+08 & 3.098 \mathrm{E}+08\end{array}$ $\begin{array}{llllllll}2.848 E+08 & 2.688 E+08 & 2.648 E+08 & 2.538 E+08 & 2.472 E+08 & 2.291 E+08 & 2.219 E+08 & 2.093 E+08\end{array}$

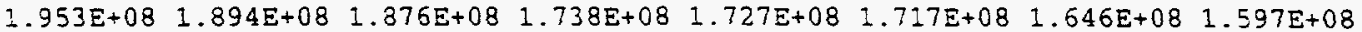

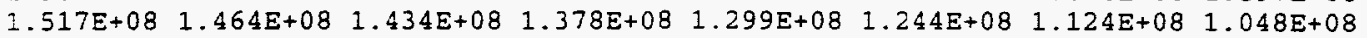
$9.788 \mathrm{E}+07 \quad 9.018 \mathrm{E}+07 \quad 8.189 \mathrm{E}+07 \quad 7.589 \mathrm{E}+07$

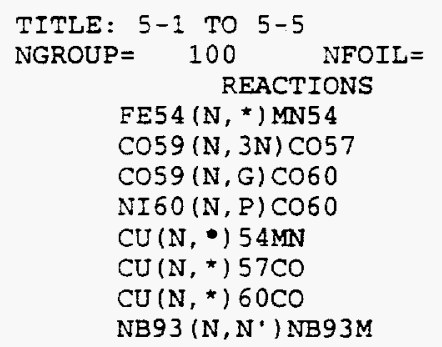

\begin{tabular}{lrrrr}
8 & ACTIVITY NORM= & $3.255 E+00$ & \\
COVER & ACTIVITIES & \multicolumn{2}{c}{ SELF-SHIELDING } \\
.00 & $5.950 E-13$ & & & .00 \\
.00 & $5.367 E-14$ & & .00 \\
.00 & $5.041 E-11$ & IFX & CONG & 1.96 \\
.00 & $3.792 E-14$ & & & .00 \\
.00 & $2.852 \mathrm{E}-15$ & & & .00 \\
.00 & $5.637 \mathrm{E}-15$ & & & .00 \\
.00 & $1.307 \mathrm{E}-14$ & & .00 \\
.00 & $5.065 \mathrm{E}-12$ & & & .00
\end{tabular}

\section{COVARIANCE PARAMETERS}

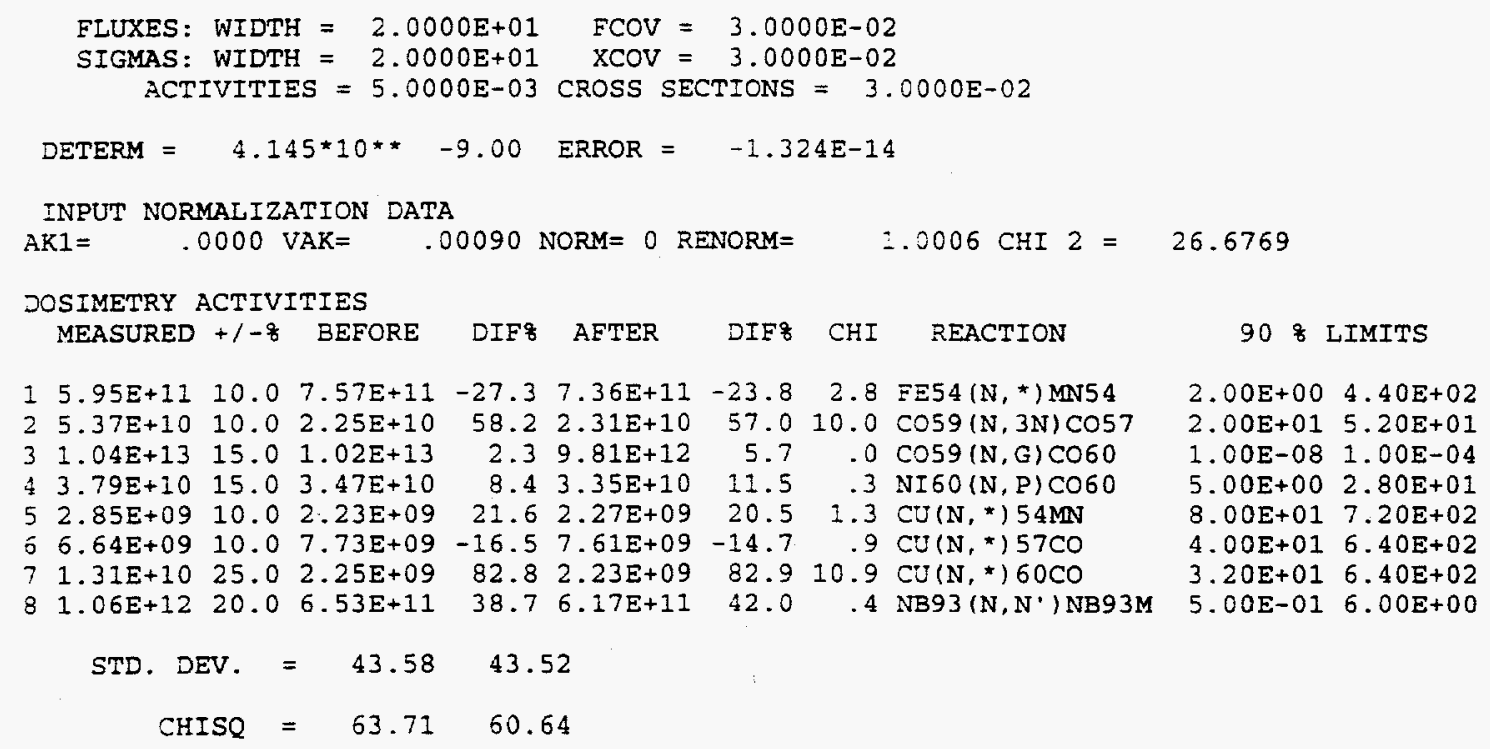

CHECK INPUT IT IS RATHER UNLIKELY $! ! !$

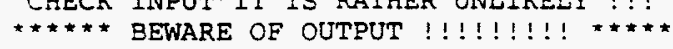




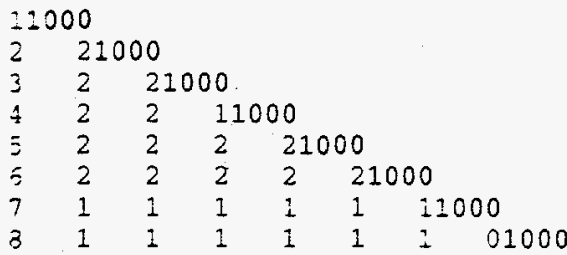

RELATIVE COV. MATRIX OF ACTIVITIES

\% CORRELATION MATRIX

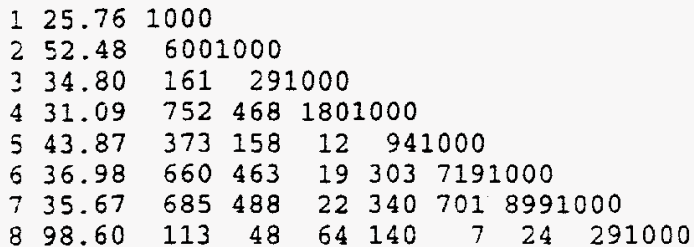

CONTRIBUTION DUE TO INPUT FLUX COV. MATRIX के CORRELATION MATRIX

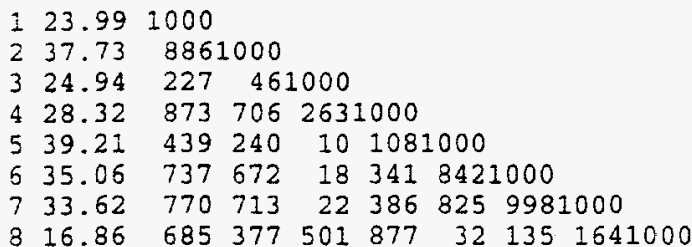

CONTRIBUTION DUE TO INPUT X-SEC. COV. MATRIX \& CORRELATION MATRIX

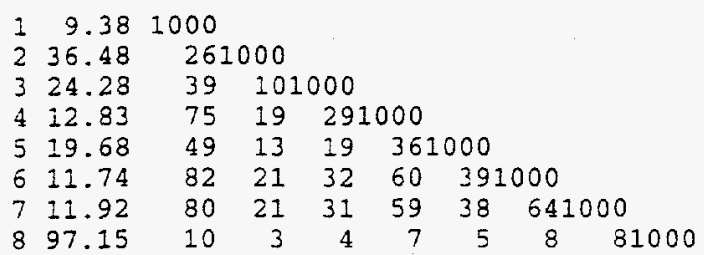

DIFFERENTIAL FLUXES INPUT NORMALIZED BY

$\begin{array}{lllr}1.000 E-10 & 1.062 \mathrm{E}+18 & 1.040 \mathrm{E}+18 & 1.022 \\ 1.000 \mathrm{E}-09 & 3.461 \mathrm{E}+17 & 3.401 \mathrm{E}+17 & 1.018 \\ 1.000 \mathrm{E}-08 & 4.085 \mathrm{E}+17 & 4.031 \mathrm{E}+17 & 1.013 \\ 2.300 \mathrm{E}-08 & 2.234 \mathrm{E}+17 & 2.214 \mathrm{E}+17 & 1.009 \\ 5.000 \mathrm{E}-08 & 1.628 \mathrm{E}+17 & 1.622 \mathrm{E}+17 & 1.004 \\ 1.150 \mathrm{E}-07 & 1.199 \mathrm{E}+17 & 1.201 \mathrm{E}+17 & .998 \\ 2.550 \mathrm{E}-07 & 8.220 \mathrm{E}+16 & 8.282 \mathrm{E}+16 & .993 \\ 5.500 \mathrm{E}-07 & 4.883 \mathrm{E}+16 & 4.948 \mathrm{E}+16 & .987 \\ 1.275 \mathrm{E}-06 & 3.269 \mathrm{E}+16 & 3.331 \mathrm{E}+16 & .981 \\ 2.800 \mathrm{E}-06 & 1.975 \mathrm{E}+16 & 2.023 \mathrm{E}+16 & .976 \\ 6.300 \mathrm{E}-06 & 9.978 \mathrm{E}+15 & 1.028 \mathrm{E}+16 & .971 \\ 1.350 \mathrm{E}-05 & 6.132 \mathrm{E}+15 & 6.347 \mathrm{E}+15 & .966 \\ 3.000 \mathrm{E}-05 & 3.291 \mathrm{E}+15 & 3.422 \mathrm{E}+15 & .962 \\ 6.900 \mathrm{E}-05 & 2.350 \mathrm{E}+15 & 2.454 \mathrm{E}+15 & .957 \\ 1.000 \mathrm{E}-04 & 1.152 \mathrm{E}+15 & 1.209 \mathrm{E}+15 & .953 \\ 2.800 \mathrm{E}-04 & 5.679 \mathrm{E}+14 & 5.973 \mathrm{E}+14 & .951 \\ 5.750 \mathrm{E}-04 & 3.966 \mathrm{E}+14 & 4.181 \mathrm{E}+14 & .949 \\ 1.275 \mathrm{E}-03 & 2.247 \mathrm{E}+14 & 2.372 \mathrm{E}+14 & .947 \\ 2.000 \mathrm{E}-03 & 1.369 \mathrm{E}+14 & 1.447 \mathrm{E}+14 & .946 \\ 3.400 \mathrm{E}-03 & 1.140 \mathrm{E}+14 & 1.205 \mathrm{E}+14 & .946 \\ 5.500 \mathrm{E}-03 & 6.951 \mathrm{E}+13 & 7.345 \mathrm{E}+13 & .946 \\ 9.200 \mathrm{E}-03 & 6.622 \mathrm{E}+13 & 6.994 \mathrm{E}+13 & .947 \\ 1.200 \mathrm{E}-02 & 2.740 \mathrm{E}+13 & 2.878 \mathrm{E}+13 & .952 \\ 5.250 \mathrm{E}-02 & 1.889 \mathrm{E}+13 & 1.968 \mathrm{E}+13 & .960 \\ 1.000 \mathrm{E}-01 & 1.269 \mathrm{E}+13 & 1.317 \mathrm{E}+13 & .964 \\ 2.000 \mathrm{E}-01 & 7.646 \mathrm{E}+12 & 7.904 \mathrm{E}+12 & .967 \\ 5.000 \mathrm{E}-01 & 3.084 \mathrm{E}+12 & 3.181 \mathrm{E}+12 & .969 \\ 1.000 \mathrm{E}+00 & 1.203 \mathrm{E}+12 & 1.255 \mathrm{E}+12 & .959\end{array}$

1.0006

STD DEV \& NEW

39.89

37.13

35.15

33.91

32.60

30.71

28.48

26.41

24.51

22.81

21.30

19.98

18.74

17.87

16.95

15.88

14.92

13.98

13.13

12.29

11.50

10.82

9.35

7.72

6.88

6.15

5.75

7.38

\subsection{8}

43.59

41.96

41.15

40.22

38.49

36.24

34.06

32.00

30.10

28.35

26.77

25.25

24.17

22.99

21.59

20.33

19.10

18.00

16.92

15.94

15.14

13.20

11.01

9.93

8.98

8.54

11.47
OLD

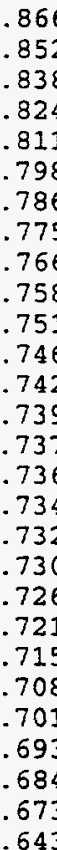

.856

$.230 E+13$

1. $230 E+13$

$1.230 \mathrm{E}+13$

1. $229 E+13$

1. $229 \mathrm{E}+13$

1. $228 E+13$

1. $226 E+13$

$1.224 \mathrm{E}+13$

1. $220 E+13$

1. $215 \mathrm{E}+13$

1. $208 \mathrm{E}+13$

1. $201 E+13$

1. $191 \mathrm{E}+13$

1. $178 \mathrm{E}+13$

1. $171 \mathrm{E}+13$

1. $150 \mathrm{E}+13$

1. $133 \mathrm{E}+13$

1. $105 \mathrm{E}+13$

1. $089 E+13$

$1.070 E+13$

$1.046 E+13$

$1.020 E+13$

1. $002 \mathrm{E}+13$

$8.909 E+12$

$8.011 E+12$

$6.743 E+12$

4. $449 E+12$

$2.907 \mathrm{E}+12$
INT FLUX $>+-8$

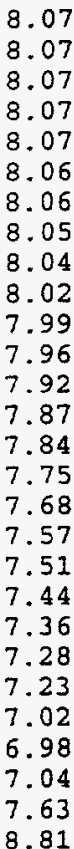

8.07

8.07

8.06

8.06

8.05

7.99

7.96

.84

68

7.51

7. 44

.23

7.02

6.98

8.81 


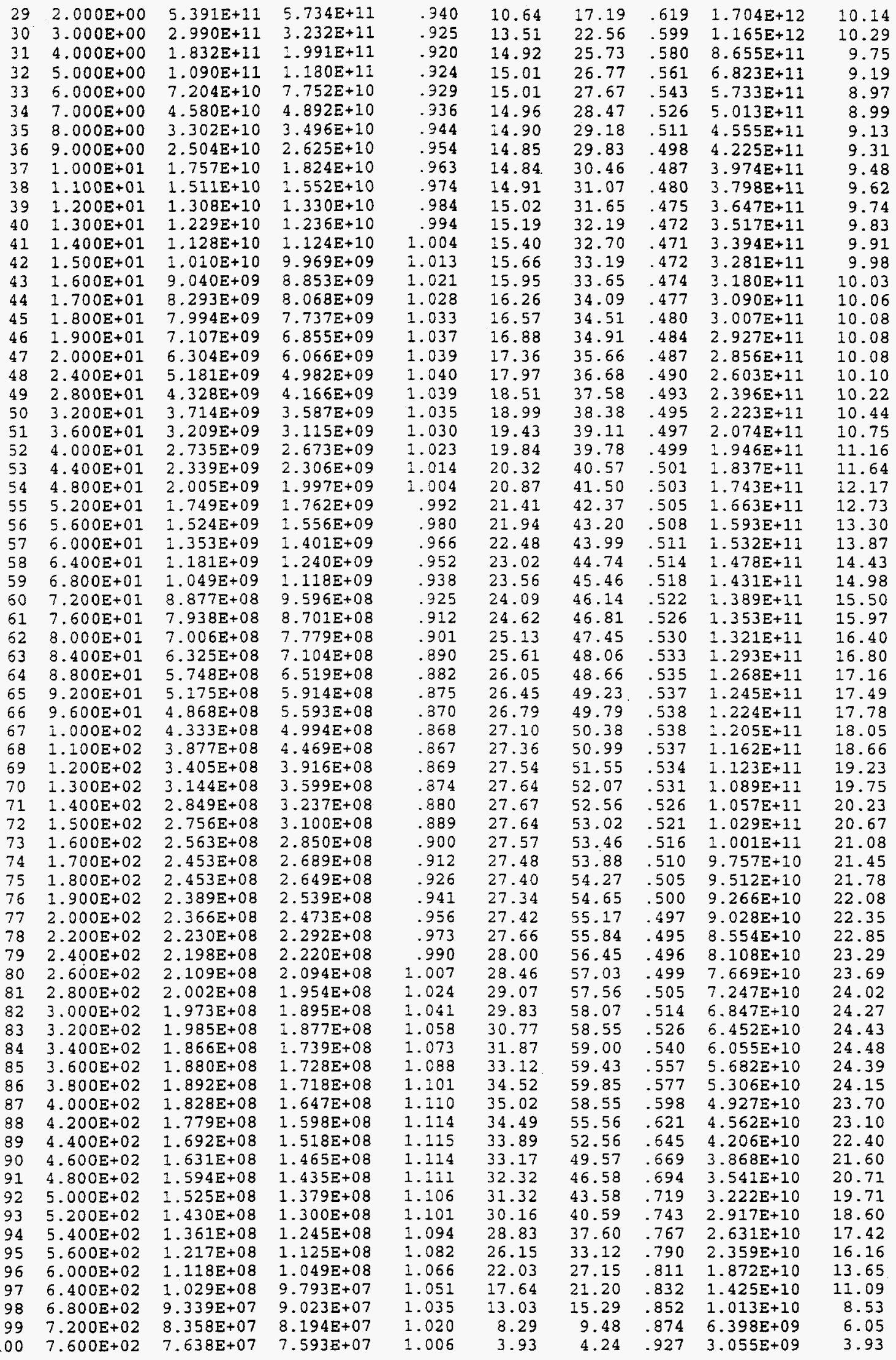

INTEGRALS OF SPECTRA

OLD SPECTRUM $1.283 E+13+$ OR- $12.495 \%$

NEW SPECTRUM $1.230 E+13$ +OR- 8.0698

$\begin{array}{llll}\text { ENERGY FLUX SNEV } & \text { FNERGY }\end{array}$ 


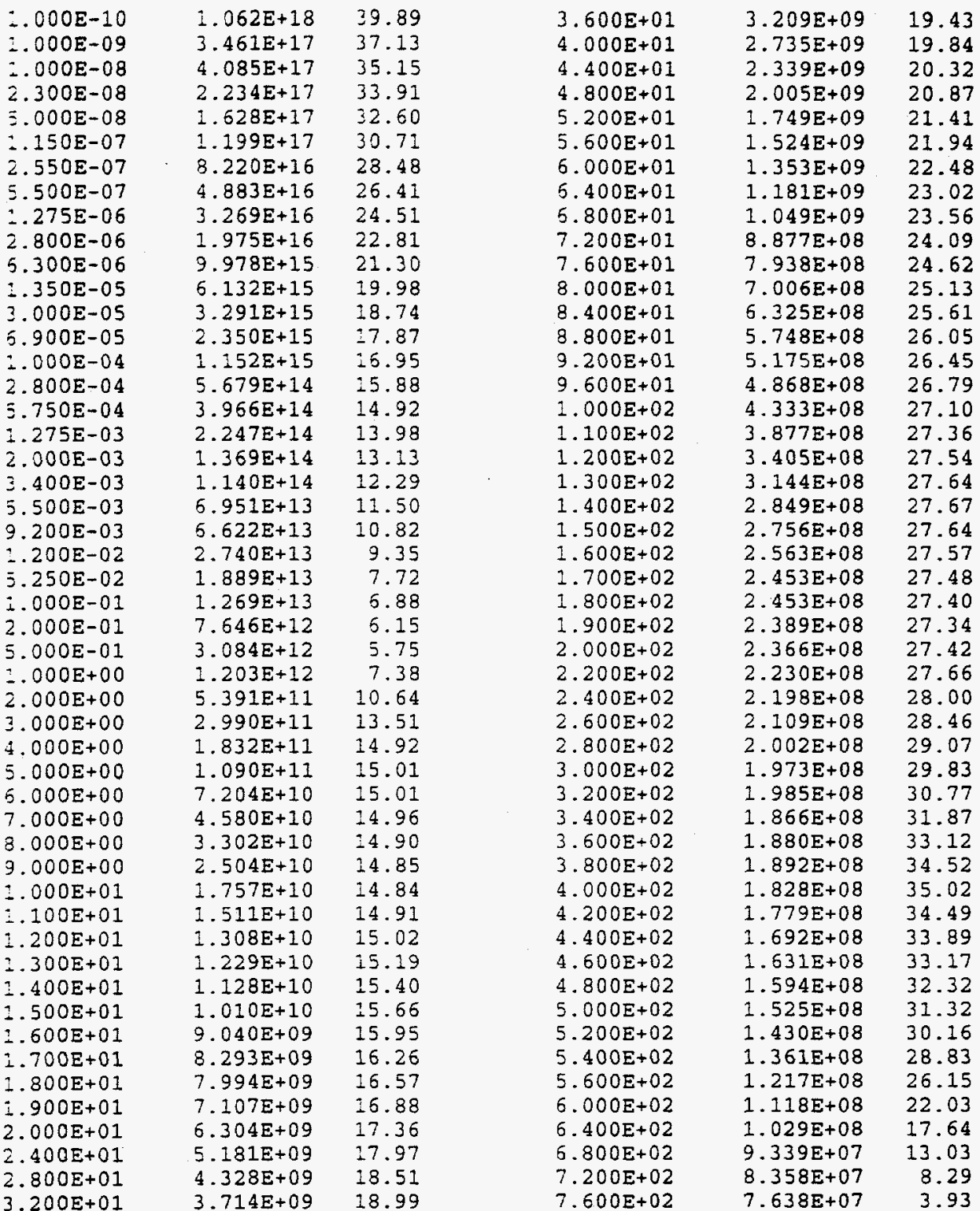

SUMMARY OF BROAD-GROUP FLUXES, FLUENCES, AND UNCERTAINTIES

\begin{tabular}{lccc} 
IRRAD TIME $(S)=6.336 E+06$ & ACT NORM $=$ & $3.255 E+00$ \\
\multicolumn{1}{l}{ ENERGY } & FLUX & FLUENCE & SDEV \\
TOTAL & $1.230 E+13$ & $2.395 E+19+1-8.07$ \\
& & & \\
$1.000 E-10$ & $2.599 E+10$ & $5.060 E+16+1-34.08$ \\
$1.150 E-07$ & $2.673 E+11$ & $5.202 E+17+1-23.99$ \\
$1.350 E-05$ & $3.998 E+12$ & $7.783 E+18+1-11.01$ \\
$1.000 E-01$ & $5.105 E+12$ & $9.937 E+18+1-6.20$ \\
$1.000 E+00$ & $2.509 E+12$ & $4.884 E+18+1-10.01$ \\
$1.000 E+01$ & $1.119 E+11$ & $2.177 E+17+1-14.66$ \\
$2.000 E+01$ & $9.094 E+10$ & $1.770 E+17+1-18.08$ \\
$4.000 E+01$ & $7.411 E+10$ & $1.443 E+17+1-20.43$ \\
$1.000 E+02$ & $3.022 E+10$ & $5.883 E+16+1-26.14$ \\
$2.000 E+02$ & $8.014 E+10$ & $1.560 E+17+1-24.56$
\end{tabular}

SUMMARY OE INTEGRATED GROUP ENERGY FLUXES AND FLUENCES

$\begin{array}{ccccrc}\text { ENERGY } & \text { FLUX } & \text { FLUX } & \text { FLUENCE } & \text { STANDARD } \\ \mathrm{MeV} & \mathrm{n} / \mathrm{cm} 2-\mathrm{s}-\mathrm{mA} & \mathrm{n} / \mathrm{cm} 2-\mathrm{s} & \mathrm{n} / \mathrm{cm} 2 & \text { EEVIATION }\end{array}$




$<1.0$
$0.1-1.0$
$1.0-10.0$
$10.0-20.0$
$20.0-40.0$
$40.0-100.0$
$100.0-200.0$
$>200.0$
TOTAL

$$
\begin{array}{ll}
4.292 \mathrm{E}+12 & -.318 \mathrm{E}+12 \\
5.105 \mathrm{E}+12 & 1.568 \mathrm{1}+12 \\
2.509 \mathrm{E}+12 & 7.709 \mathrm{E}+11 \\
1.119 \mathrm{E}+11 & 3.437 \mathrm{E}+10 \\
9.094 \mathrm{E}+10 & 2.794 \mathrm{E}+10 \\
7.411 \mathrm{E}+10 & 2.277 \mathrm{E}+10 \\
3.022 \mathrm{E}+10 & 9.285 \mathrm{E}+09 \\
8.014 \mathrm{E}+10 & 2.462 \mathrm{E}+10 \\
1.230 \mathrm{E}+13 & 3.780 \mathrm{E}+12
\end{array}
$$

$.354 E+18$ $9.937 E+18$ $4.884 E+18$
$-1-11.01$

$+1-6.20$

$+/-10.01$

$-1-14.66$

$+/-18.08$

$+1-20.43$

$+1-26.14$

$+1-24.56$

$-1-8.07$
34.88

41.49

20.39

$2.395 E+19$

THE PERCENTAGE OF NEUTRONS ABOVE $20 \mathrm{MeV}$ IS - 2.24

RELATIVE COVARIANCES (10X10)

$\begin{array}{rrrrrrrrr}1000 & 906 & 410 & 130 & 52 & -15 & 4 & 10 & 6 \\ 1000 & 696 & 357 & 238 & -38 & -59 & -25 & 14 & 16 \\ 1000 & 880 & 753 & 38 & -229 & -167 & 32 & 54 & \\ 1000 & 933 & 207 & -237 & -237 & 20 & 76 & & \\ 1000 & 433 & -154 & -271 & -12 & 83 & & & \\ 1000 & 673 & 128 & -250 & -61 & & & & \\ 1000 & 692 & -226 & -303 & & & & & \\ 1000 & 283 & -488 & & & & & & \\ 1000 & 27 & & & & & & & \\ 1000 & & & & & & & \end{array}$




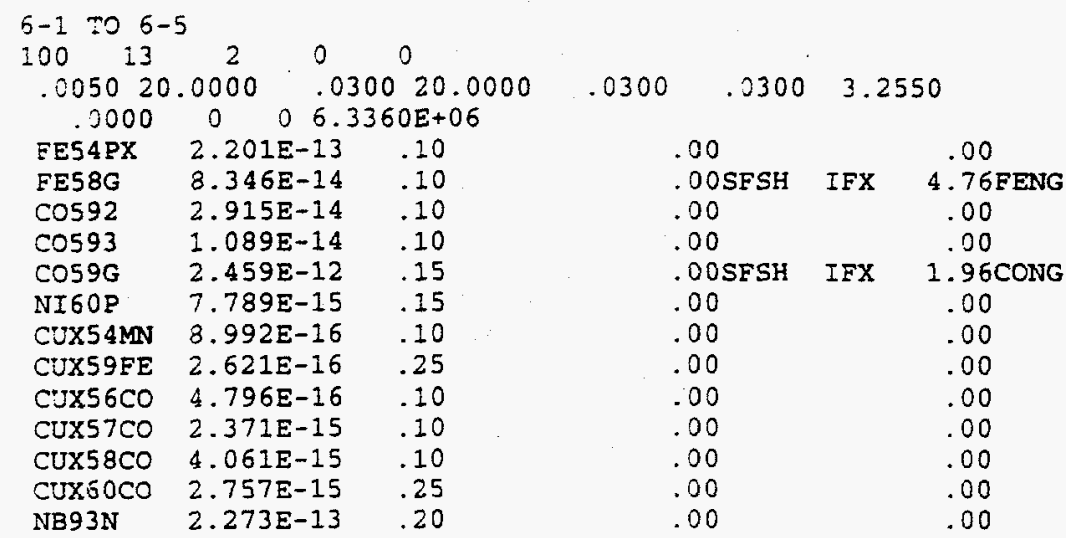

0 ERACTIONAL ERRORS IN INPUT SPECTRUM

$161.000 E+00$

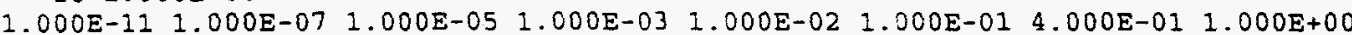

$\begin{array}{llllllll}4.000 E+00 & 1.000 E+01 & 2.000 E+01 & 4.400 E+01 & 1.000 E+02 & 4.000 E+02 & 8.000 E+02 & 0.000 E+00\end{array}$

5.000E-01 4.000E-01 2.800E-01 2.000E-01 1.500E-01 1.000E-01 8.000E-02 8.000E-02

$2.500 \mathrm{E}-013.000 \mathrm{E}-013.500 \mathrm{E}-01 \quad 4.000 \mathrm{E}-01 \quad 5.000 \mathrm{E}-01 \quad 6.000 \mathrm{E}-01 \quad 0.000 \mathrm{E}+00 \quad 0.000 \mathrm{E}+00$

1 INPUT SPECTRUM

$1001.000 E+00$

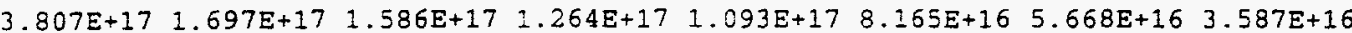

$2.189 \mathrm{E}+16 \quad 1.373 \mathrm{E}+16 \quad 7.049 \mathrm{E}+15 \quad 3.772 \mathrm{E}+15 \quad 2.262 \mathrm{E}+15 \quad 1.413 \mathrm{E}+15 \quad 9.417 \mathrm{E}+14 \quad 4.708 \mathrm{E}+14$

$2.446 \mathrm{E}+14 \quad 1.634 \mathrm{E}+14 \quad 1.299 \mathrm{E}+14 \quad 9.805 \mathrm{E}+13 \quad 7.778 \mathrm{E}+13 \quad 7.036 \mathrm{E}+13 \quad 3.239 \mathrm{E}+13 \quad 1.827 \mathrm{E}+13$

$\begin{array}{llllllll}1.506 \mathrm{E}+13 & 7.484 \mathrm{E}+12 & 3.313 \mathrm{E}+12 & 1.455 \mathrm{E}+12 & 7.209 \mathrm{E}+11 & 3.507 \mathrm{E}+11 & 1.741 \mathrm{E}+11 & 6.522 \mathrm{E}+10\end{array}$

$3.996 E+10 \quad 2.991 E+10 \quad 2.362 E+10 \quad 1.861 E+10 \quad 1.533 E+10 \quad 1.329 E+10 \quad 1.137 E+10 \quad 9.749 E+09$

$9.116 E+09 \quad 8.719 E+09 \quad 8.355 E+09 \quad 8.020 E+09 \quad 7.749 E+09 \quad 7.518 E+09 \quad 6.677 E+09 \quad 5.677 E+09$

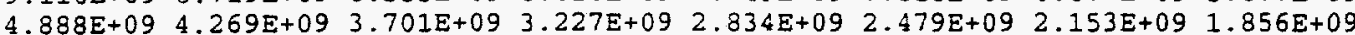

$\begin{array}{llllllll}1.605 E+09 & 1.358 E+09 & 1.166 E+09 & 9.801 E+08 & 8.572 E+08 & 7.813 E+08 & 6.823 E+08 & 6.208 E+08\end{array}$

$\begin{array}{llllllll}5.492 E+08 & 4.895 E+08 & 4.343 E+08 & 3.859 E+08 & 3.477 E+08 & 3.274 E+08 & 3.036 E+08 & 2.933 E+08\end{array}$

$\begin{array}{lllllllll}2.814 E+08 & 2.770 E+08 & 2.747 E+08 & 2.790 E+08 & 2.642 E+08 & 2.507 E+08 & 2.348 E+08 & 2.250 E+08\end{array}$

$\begin{array}{llllllll}2.131 E+08 & 1.994 \mathrm{E}+08 & 1.873 \mathrm{E}+08 & 1.828 \mathrm{E}+08 & 1.729 \mathrm{E}+08 & 1.612 \mathrm{E}+08 & 1.587 \mathrm{E}+08 & 1.513 \mathrm{E}+08\end{array}$

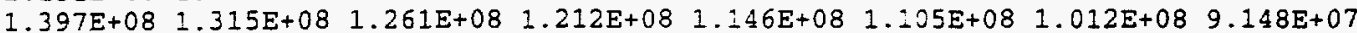

$8.162 E+07 \quad 7.436 E+07 \quad 6.796 E+07 \quad 6.250 E+07$

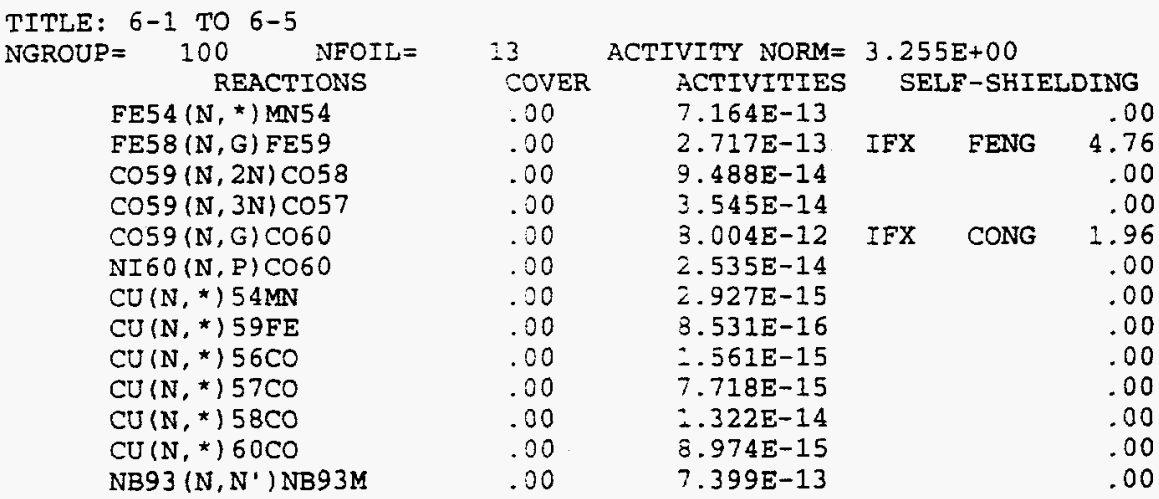

\section{COVARIANCE PARAMETERS}

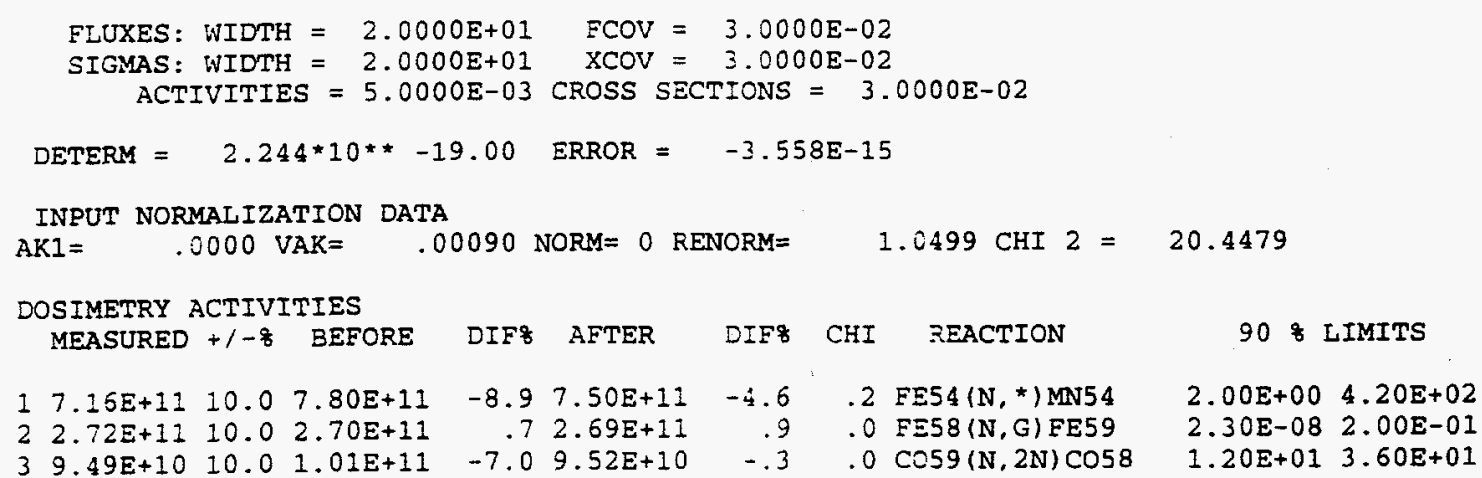


$43.54 \mathrm{E}+10 \quad 10.0 \quad 2.77 \mathrm{E}+10$

$58.00 \mathrm{E}+1215.0 \quad 7.80 \mathrm{E}+12$

$\begin{array}{llll}6 & 2.54 \mathrm{E}+10 & 15.0 & 2.78 \mathrm{E}+10\end{array}$

$72.93 E+0910.022 .26 E+09$

$88.53 E+0825.0 \quad 1.84 E+08$

$1.56 \mathrm{E}+0910.01 .69 \mathrm{E}+09$

$107.72 \mathrm{E}+09 \quad 10.0 \quad 8.50 \mathrm{E}+09$

$11 \quad 1.32 E+10 \quad 10.0 \quad 1.33 E+10$

$128.97 E+0925.0 \quad 2.49 E+09$

$137.40 \mathrm{E}+1120.0 \quad 7.33 \mathrm{E}+11$
$22.02 .59 \mathrm{E}+10$ $2.5 \quad 7.84 \mathrm{E}+12$ $\begin{array}{ll}-9.8 & 2.62 \mathrm{E}+10\end{array}$ $22.8 \quad 2.49 \mathrm{E}+09$

$78.51 .98 \mathrm{E}+08$ $-.91 .31 \mathrm{E}+10$ $72.32 .50 E+09$ $97.08 \mathrm{E}+11$
27.0

2.1

$-3.2$

15.1

$-10.6$

72.2

4.3
$.7 \cos 9(\mathrm{~N}, 3 \mathrm{~N}) \cos 7$

$.0 \operatorname{Cos} 9(\mathrm{~N}, \mathrm{G}) \operatorname{Cos} 0$

$.1 \mathrm{NI60}$ (N, P) C060

$1.1 \mathrm{CU}(\mathrm{N}, *) 54 \mathrm{MN}$

$9.4 \mathrm{CU}(\mathrm{N}, *) 59 \mathrm{FE}$

$.3 \mathrm{CU}(\mathrm{N}, *) 56 \mathrm{CO}$

$.4 \mathrm{CU}(\mathrm{N}, *) 57 \mathrm{CO}$

$.0 \mathrm{CU}(\mathrm{N}, *) 58 \mathrm{CO}$

$8.2 \mathrm{CU}\left(\mathrm{N},{ }^{\star}\right) 60 \mathrm{CO}$

. 0 NB93 (N, N') NB93
$2.00 E+01 \quad 5.20 E+01$ $2.30 \mathrm{E}-08 \quad 1.00 \mathrm{E}-04$ $5.00 E+00 \quad 4.00 E+01$ $7.60 \mathrm{E}+01 \quad 6.80 \mathrm{E}+02$ $5.60 \mathrm{E}+016.80 \mathrm{E}+02$ $6.40 \mathrm{E}+01 \quad 6.40 \mathrm{E}+02$ $4.00 \mathrm{E}+01 \quad 6.00 \mathrm{E}+02$ $2.80 E+01 \quad 5.40 E+02$ $3.20 \mathrm{E}+01 \quad 6.00 \mathrm{E}+02$

$$
\text { CHISQ }=31.62 \quad 30.77
$$

DOSIMETRY DATA INPUT CORRELATION MATRIX

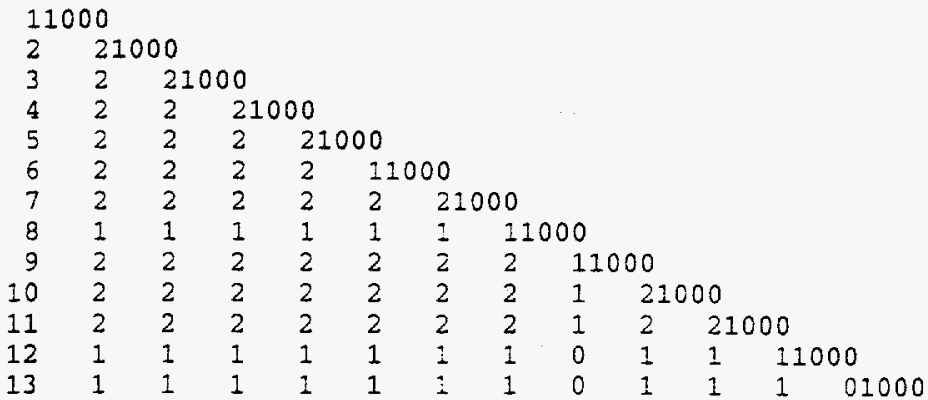

RELATIVE COV. MATRIX OF ACTIVITIES

$$
\text { के }
$$

$126.70 \quad 1000$

$221.54 \quad 2441000$

$3 \begin{array}{lll}35.76 & 813 \quad 1531000\end{array}$

$452.43 \quad 620 \quad 62 \quad 6471000$

$\begin{array}{llllll}5 & 34.88 & 144 & 656 & 79 & 291000\end{array}$

$\begin{array}{lllllll}6 & 31.22 & 740 & 298 & 794 & 498 & 1711000\end{array}$

$\begin{array}{llllllll}7 & 44.59 & 386 & 22 & 137 & 162 & 12 & 1141000\end{array}$

$\begin{array}{lllllllll}8 & 41.74 & 459 & 26 & 208 & 230 & 14 & 159 & 8181000\end{array}$

$9 \begin{array}{llllllllll}9 & 42.38 & 505 & 25 & 243 & 272 & 13 & 176 & 819 & 8471000\end{array}$

$\begin{array}{lllllllllll}10 & 36.94 & 711 & 42 & 510 & 492 & 20 & 362 & 707 & 771 & 8251000\end{array}$

$\begin{array}{llllllllllll}11 & 35.35 & 802 & 58 & 669 & 600 & 28 & 492 & 583 & 661 & 720 & 8881000\end{array}$

$\begin{array}{lllllllllllll}12 & 35.72 & 736 & 48 & 555 & 519 & 23 & 402 & 684 & 750 & 802 & 906 & 8951000\end{array}$

$\begin{array}{llllllllllllll}13 & 99.30 & 96 & 100 & 90 & 44 & 63 & 127 & 7 & 10 & 10 & 24 & 38 & 291000\end{array}$

CONTRIBUTION DUE TO INPUT FLUX COV. MATRIX

के

CORRELATION MATRIX

$\begin{array}{rl}1 & 24.79 \\ 2 & 20.13 \\ 3 & 33.78 \\ 4 & 37.82 \\ 5 & 24.32 \\ 6 & 28.47 \\ 7 & 40.15 \\ 8 & 37.99 \\ 9 & 39.56 \\ 10 & 35.18 \\ 11 & 33.79 \\ 12 & 33.82 \\ 13 & 16.18\end{array}$

1000

2641000

9161611000

917819421000

$207993108 \quad 491000$

$861336913749 \quad 2561000$

$\begin{array}{llllll}453 & 15 & 155 & 244 & 10 & 1311000\end{array}$

$\begin{array}{lllllll}534 & 19 & 235 & 344 & 12 & 183 & 9921000\end{array}$

$\begin{array}{llllllll}574 & 18 & 269 & 398 & 11 & 199 & 969 & 9911000\end{array}$

$\begin{array}{lllllllll}794 & 34 & 559 & 709 & 20 & 408 & 818 & 883 & 9221000\end{array}$

$\begin{array}{llllllllll}893 & 52 & 733 & 863 & 31 & 555 & 670 & 753 & 801 & 9681000\end{array}$

$\begin{array}{lllllllllll}827 & 41 & 612 & 752 & 24 & 457 & 796 & 863 & 900 & 997 & 9811000\end{array}$

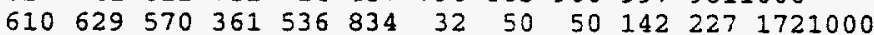

CONTRIBUTION DUE TO INPUT X-SEC. COV. MATRIX

\&

CORRELATION MATRIX

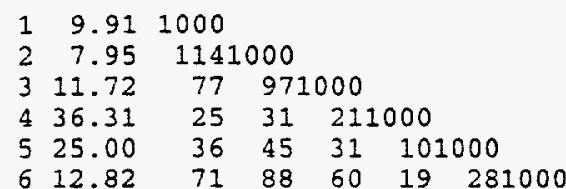



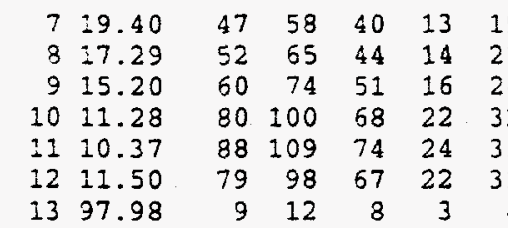

9 361000

141271000

$\begin{array}{llll}4 & 46 & 31 & 341000\end{array}$

$\begin{array}{lllll}35 & 62 & 41 & 46 & 531000 \\ 38 & 45 & 50 & 57 & 77100\end{array}$

1211.50

$\begin{array}{lllllllllll}79 & 98 & 57 & 22 & 31 & 61 & 40 & 45 & 52 & 69 & 761000\end{array}$

DIFFERENTIAL FIUXES INPUT NORMALIZED BY

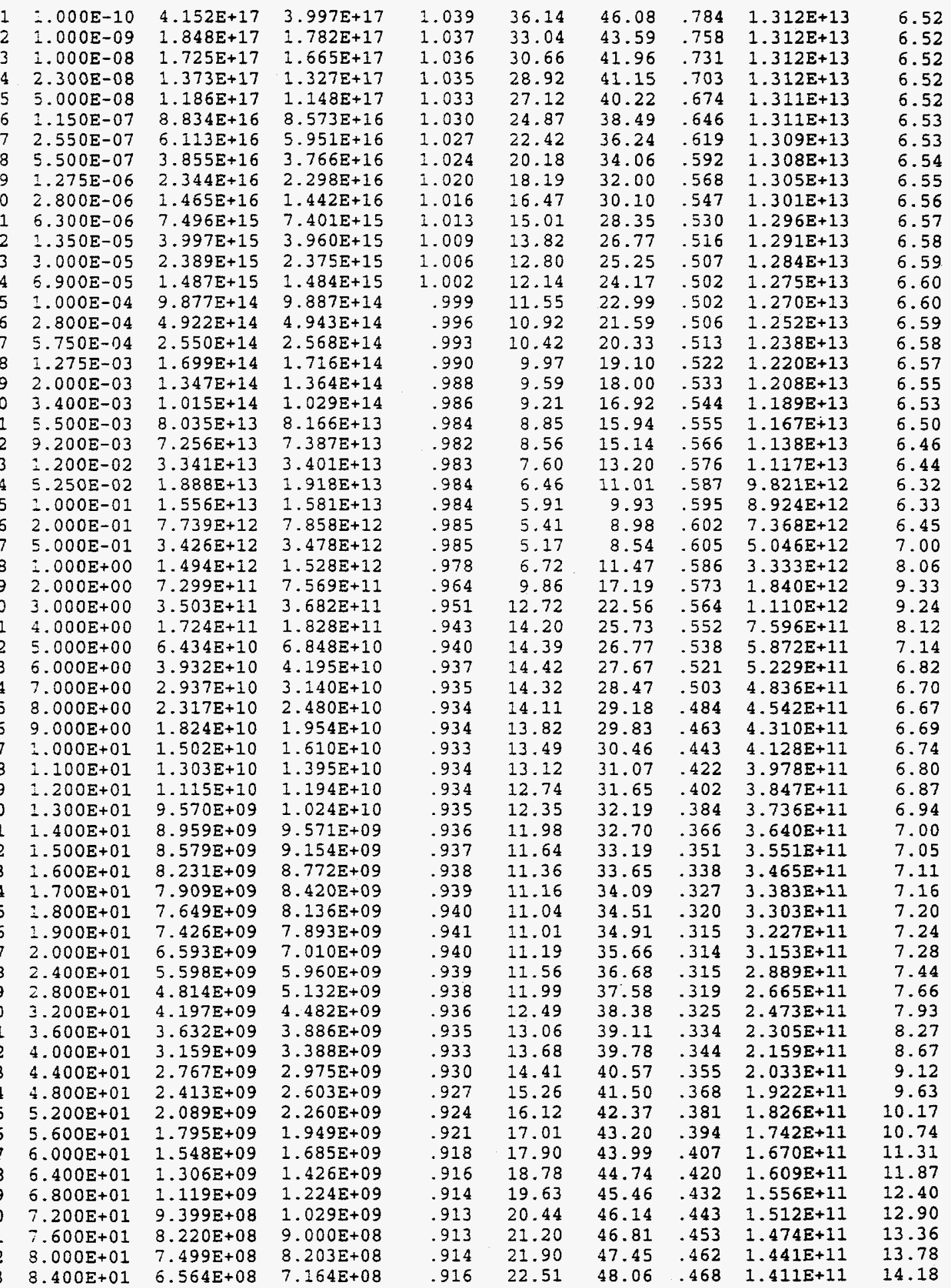




\begin{tabular}{|c|c|c|c|c|c|c|c|c|c|}
\hline 64 & $8.800 E+01$ & $5.994 E+08$ & $6.518 E+08$ & .920 & 23.04 & 48.66 & .474 & $1.385 \mathrm{E}+11$ & 14.54 \\
\hline & $9.200 \mathrm{E}+01$ & $5.330 \mathrm{E}+08$ & $5.766 E+08$ & .924 & 23.47 & 49.23 & .477 & $361 E+11$ & 14.88 \\
\hline 66 & $9.600 \mathrm{E}+01$ & $4.783 E+08$ & $5.139 E+08$ & .931 & 23.80 & 49.79 & .478 & $1.340 E+11$ & 15.18 \\
\hline 67 & $1.000 \mathrm{E}+02$ & $4.278 \mathrm{E}+08$ & $4.560 E+08$ & .938 & 24.03 & 50.38 & .477 & $1.320 E+11$ & 15.45 \\
\hline 68 & 1. $100 E+02$ & $3.838 E+08$ & $4.052 E+08$ & .947 & 24.18 & 50.99 & .474 & $1.278 \mathrm{E}+11$ & \\
\hline 69 & $1.200 \mathrm{E}+02$ & $3.497 E+08$ & $3.651 E+08$ & .958 & 24.21 & 51.55 & .470 & $1.239 \mathrm{E}+11$ & .64 \\
\hline 70 & $1.300 \mathrm{E}+02$ & $3.232 E+08$ & $3.332 E+08$ & .970 & 24.12 & 52.07 & .463 & $1.204 \mathrm{E}+11$ & 17.18 \\
\hline 71 & $1.400 E+02$ & $E+08$ & $3 E+08$ & .983 & 23.95 & 52.56 & .456 & $1.172 E+11$ & 17.68 \\
\hline 72 & $1.500 E+02$ & $3.073 E+08$ & $3.079 E+08$ & 998 & 23.70 & 53.02 & .447 & $1.141 E+11$ & 18.17 \\
\hline 73 & $1.600 E+02$ & $2.995 E+08$ & $2.954 E+08$ & 1.014 & 23.41 & 53.46 & .438 & $1.110 E+11$ & 18.64 \\
\hline 74 & $1.700 E+02$ & $2.996 E+08$ & $2.908 \mathrm{E}+08$ & 1.030 & 23.11 & 53.88 & .429 & $1.080 E+11$ & 19.09 \\
\hline 75 & $1.800 E+02$ & $3.021 E+08$ & $4 E+08$ & 1.047 & 22.83 & 54.27 & .421 & $1.050 E+11$ & 19.52 \\
\hline 76 & $1.900 \mathrm{E}+02$ & $3.119 E+08$ & $2.929 E+08$ & 1.065 & 22.63 & 54.65 & .414 & $E+11$ & .93 \\
\hline 77 & $2.000 E+02$ & $3.004 \mathrm{E}+08$ & $2.774 \mathrm{E}+08$ & 1.083 & 22.60 & 55.17 & .410 & $9.886 E+10$ & 20.32 \\
\hline 78 & $2.200 E+02$ & $2.899 E+08$ & $2.632 \mathrm{E}+08$ & 1.102 & 22.78 & 84 & .408 & $5 E+10$ & 1.05 \\
\hline 79 & $2.400 E+02$ & $2.761 E+08$ & $2.465 E+08$ & 1.120 & 23.14 & 56.45 & .410 & $5 E+10$ &. .74 \\
\hline 80 & $2.600 E+02$ & $2.688 E+08$ & $2.352 E+08$ & 1.138 & 23.71 & 57.03 & .416 & $E+10$ & 22.38 \\
\hline 81 & $2.800 E+02$ & $2.585 E+08$ & $2.237 \mathrm{E}+08$ & 1.155 & 24.51 & 57.56 & .426 & $5 E+10$ & 22.95 \\
\hline 82 & $3.000 E+02$ & $2.454 \mathrm{E}+08$ & $2.094 \mathrm{E}+08$ & 1.172 & 25.54 & 58.07 & .440 & $E+10$ & .44 \\
\hline 83 & $3.200 E+02$ & $2.336 E+08$ & $1.967 \mathrm{E}+08$ & 1.188 & 25.80 & 58.55 & .458 & +10 & 23.82 \\
\hline 84 & $3.400 \mathrm{E}+02$ & $2.307 \mathrm{E}+08$ & $1.919 E+08$ & 1.202 & 28.26 & 59.00 & .479 & $6.140 E+10$ & 24.06 \\
\hline 85 & $3.600 \mathrm{E}+02$ & $2.206 E+08$ & $1.815 E+08$ & 1.215 & 29.90 & 59.43 & .503 & $E+10$ & 24.14 \\
\hline 86 & $3.800 E+02$ & 2.07 & $2 E+08$ & 1.227 & 31.69 & 85 & .529 & 10 & .04 \\
\hline 87 & $4.000 E+02$ & 2.049 & $1.666 E+08$ & 1.230 & 32.63 & 58.55 & .557 & $E+10$ & 23.72 \\
\hline 88 & $4.200 E+02$ & $1.945 \mathrm{E}+08$ & $1.589 \mathrm{E}+08$ & 1.224 & 32.58 & 55.56 & .586 & $4.413 E+10$ & 23.19 \\
\hline 89 & $4.400 E+02$ & $1.784 E+08$ & $1.467 \mathrm{E}+08$ & 1.216 & 32.37 & 52.56 & .616 & $4 E+10$ & 22.54 \\
\hline 90 & 4.60 & 1.666 & 2. $381 E+08$ & 1.207 & 32.00 & 49.57 & .646 & +10 & 21.80 \\
\hline 91 & $4.800 E+02$ & $1.583 E+08$ & $1.324 \mathrm{E}+08$ & 1.195 & 31.44 & 46.58 & .675 & $E+10$ & 0.94 \\
\hline 92 & $5.000 E+02$ & $1.505 E+08$ & $1.273 E+08$ & 1.183 & 30.67 & 43.58 & .704 & $7 E+10$ & 19.98 \\
\hline 93 & $5.200 E+02$ & $1.407 \mathrm{E}+08$ & $1.203 E+08$ & 1.169 & 29.69 & 40.59 & .731 & $2.716 \mathrm{E}+10$ & 18.89 \\
\hline 94 & $5.400 E+02$ & $1.339 E+08$ & $1.160 \mathrm{E}+08$ & 1.155 & 28.51 & 37.60 & .758 & $2.435 \mathrm{E}+10$ & 17.72 \\
\hline 95 & $5.600 \mathrm{E}+02$ & $1.204 \mathrm{E}+08$ & $1.063 E+08$ & 1.134 & 25.95 & 33.12 & .783 & $2.167 \mathrm{E}+10$ & 16.44 \\
\hline 96 & $6.000 \mathrm{E}+02$ & $1.063 E+08$ & $9.605 E+07$ & 1.107 & 21.92 & 27.15 & .807 & $1.685 E+10$ & 13.80 \\
\hline 97 & $6.400 E+02$ & $9.260 E+07$ & $8.569 E+07$ & 1.081 & 17.59 & 21.20 & .830 & $1.260 E+10$ & 11.14 \\
\hline 98 & $6.800 E+02$ & $8.242 E+07$ & $7.807 \mathrm{E}+07$ & 1.056 & 13.01 & 15.29 & .851 & $8.895 E+09$ & 8.55 \\
\hline 95 & $7.200 E+02$ & $7.365 E+07$ & $7.235 E+07$ & 1.032 & 8.29 & 9.48 & .874 & $5.598 \mathrm{E}+09$ & 6.06 \\
\hline 100 & $7.600 E+02$ & $6.631 E+07$ & $6.562 E+07$ & 1.010 & 3.93 & 4.24 & .926 & $2.652 \mathrm{E}+09$ & 3.93 \\
\hline
\end{tabular}

INTEGRALS OF SPECTRA

$\begin{array}{lllrl}\text { OLD SPECTRUM } & 1.337 E+13 & \text { +OR- } & 12.1508 \\ \text { NEW SPECTRUM } & 1.312 E+13 \text { +OR- } & 6.5158\end{array}$

$\begin{array}{lccccc}\text { ENERGY } & \text { ELUX } & \text { SDEV } & \text { ENERGY } & \text { FLUX } & \text { SDEV } \\ 1.000 E-10 & 4.152 E+17 & 36.14 & 3.600 E+01 & 3.632 E+09 & 13.06 \\ 1.000 E-09 & 1.848 E+17 & 33.04 & 4.000 E+01 & 3.159 E+09 & 13.68 \\ 1.000 E-08 & 1.725 E+17 & 30.66 & 4.400 E+01 & 2.767 E+09 & 14.41 \\ 2.300 E-08 & 1.373 E+17 & 28.92 & 4.800 E+01 & 2.413 E+09 & 15.26 \\ 5.000 E-08 & 1.186 E+17 & 27.12 & 5.200 E+01 & 2.089 E+09 & 16.12 \\ 1.150 E-07 & 8.834 E+16 & 24.87 & 5.600 E+01 & 1.795 E+09 & 17.01 \\ 2.550 E-07 & 6.113 E+16 & 22.42 & 6.000 E+01 & 1.548 E+09 & 17.90 \\ 5.500 E-07 & 3.855 E+16 & 20.18 & 6.400 E+01 & 1.306 E+09 & 18.78 \\ 1.275 E-06 & 2.344 E+16 & 18.19 & 6.800 E+01 & 1.119 E+09 & 19.63 \\ 2.800 E-06 & 1.465 E+16 & 16.47 & 7.200 E+01 & 9.399 E+08 & 20.44 \\ 6.300 E-06 & 7.496 E+15 & 15.01 & 7.600 E+01 & 8.220 E+08 & 21.20 \\ 1.350 E-05 & 3.997 E+15 & 13.82 & 8.000 E+01 & 7.499 E+08 & 21.90 \\ 3.000 E-05 & 2.389 E+15 & 12.80 & 8.400 E+01 & 6.564 E+08 & 22.51 \\ 6.900 E-05 & 1.487 E+15 & 12.14 & 8.800 E+01 & 5.994 E+08 & 23.04 \\ 1.000 E-04 & 9.877 E+14 & 11.55 & 9.200 E+01 & 5.330 E+08 & 23.47 \\ 2.800 E-04 & 4.922 E+14 & 10.92 & 9.600 E+01 & 4.783 E+08 & 23.80 \\ 5.750 E-04 & 2.550 E+14 & 10.42 & 1.000 E+02 & 4.278 E+08 & 24.03 \\ 1.275 E-03 & 1.699 E+14 & 9.97 & 1.100 E+02 & 3.838 E+08 & 24.18 \\ 2.000 E-03 & 1.347 E+14 & 9.59 & 1.200 E+02 & 3.497 E+08 & 24.21 \\ 3.400 E-03 & 1.015 E+14 & 9.21 & 1.300 E+02 & 3.232 E+08 & 24.12 \\ 5.500 E-03 & 8.035 E+13 & 8.85 & 1.400 E+02 & 3.135 E+08 & 23.95 \\ 9.200 E-03 & 7.256 E+13 & 8.56 & 1.500 E+02 & 3.073 E+08 & 23.70 \\ 1.200 E-02 & 3.341 E+13 & 7.60 & 1.600 E+02 & 2.995 E+08 & 23.41 \\ 5.250 E-02 & 1.888 E+13 & 6.46 & 1.700 E+02 & 2.996 E+08 & 23.11 \\ 1.000 E-01 & 1.556 E+13 & 5.91 & 1.800 E+02 & 3.021 E+08 & 22.83 \\ 2.000 E-01 & 7.739 E+12 & 5.41 & 1.900 E+02 & 3.119 E+08 & 22.63 \\ 5.000 E-01 & 3.426 E+12 & 5.17 & 2.000 E+02 & 3.004 E+08 & 22.60 \\ 1.000 E+00 & 1.494 E+12 & 6.72 & 2.200 E+02 & 2.899 E+08 & 22.78 \\ 2.000 E+00 & 7.299 E+11 & 9.86 & 2.400 E+02 & 2.761 E+08 & 23.14 \\ 3.000 E+00 & 3.503 E+11 & 12.72 & 2.600 E+02 & 2.688 E+08 & 23.71\end{array}$




\begin{tabular}{|c|c|c|c|c|c|}
\hline 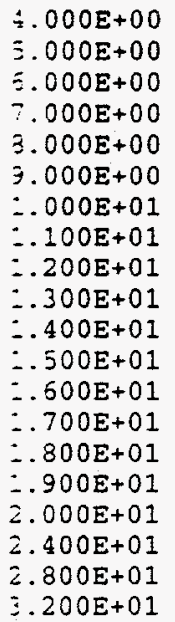 & $\begin{array}{l}1.724 E+11 \\
5.434 E+10 \\
3.932 E+10 \\
2.937 E+10 \\
2.317 E+10 \\
1.824 E+10 \\
1.502 E+10 \\
1.303 E+10 \\
1.115 E+10 \\
9.570 E+09 \\
8.959 E+09 \\
8.579 E+09 \\
8.231 E+09 \\
7.909 E+09 \\
7.649 E+09 \\
7.426 E+09 \\
6.593 E+09 \\
5.598 E+09 \\
4.814 E+09 \\
4.197 E+09\end{array}$ & $\begin{array}{l}14.20 \\
14.39 \\
14.42 \\
14.32 \\
14.11 \\
13.82 \\
13.49 \\
13.12 \\
12.74 \\
12.35 \\
11.98 \\
11.64 \\
11.36 \\
11.16 \\
11.04 \\
11.01 \\
11.19 \\
11.56 \\
11.99 \\
12.49\end{array}$ & $\begin{array}{l}2.800 \mathrm{E}+02 \\
3.000 \mathrm{E}+02 \\
3.200 \mathrm{E}+02 \\
3.400 \mathrm{E}+02 \\
3.600 \mathrm{E}+02 \\
3.800 \mathrm{E}+02 \\
4.000 \mathrm{E}+02 \\
4.200 \mathrm{E}+02 \\
4.400 \mathrm{E}+02 \\
4.600 \mathrm{E}+02 \\
4.800 \mathrm{E}+02 \\
5.000 \mathrm{E}+02 \\
5.200 \mathrm{E}+02 \\
5.400 \mathrm{E}+02 \\
5.600 \mathrm{E}+02 \\
6.000 \mathrm{E}+02 \\
6.400 \mathrm{E}+02 \\
6.800 \mathrm{E}+02 \\
7.200 \mathrm{E}+02 \\
7.600 \mathrm{E}+02\end{array}$ & $\begin{array}{l}2.585 \mathrm{E}+08 \\
2.454 \mathrm{E}+08 \\
2.336 \mathrm{E}+08 \\
2.307 \mathrm{E}+08 \\
2.206 \mathrm{E}+08 \\
2.076 \mathrm{E}+08 \\
2.049 \mathrm{E}+08 \\
1.945 \mathrm{E}+08 \\
1.784 \mathrm{E}+08 \\
1.666 \mathrm{E}+08 \\
1.583 \mathrm{E}+08 \\
1.505 \mathrm{E}+08 \\
1.407 \mathrm{E}+08 \\
1.339 \mathrm{E}+08 \\
1.204 \mathrm{E}+08 \\
1.063 \mathrm{E}+08 \\
9.260 \mathrm{E}+07 \\
8.242 \mathrm{E}+07 \\
7.365 \mathrm{E}+07 \\
6.631 \mathrm{E}+07\end{array}$ & $\begin{array}{l}24.51 \\
25.54 \\
26.80 \\
28.26 \\
29.90 \\
31.69 \\
32.63 \\
32.58 \\
32.37 \\
32.00 \\
31.44 \\
30.67 \\
29.69 \\
28.51 \\
25.95 \\
21.92 \\
17.59 \\
13.01 \\
8.29 \\
3.93\end{array}$ \\
\hline
\end{tabular}

SUMMARY OE BROAD-GROUP FLUXES, FLUENCES, AND UNCERTAINTIES

\begin{tabular}{|c|c|c|c|c|}
\hline ENERGY & ELUX & ELUENCE & & SDEV \\
\hline TOTAL & $1.312 \mathrm{E}+13$ & $2.554 \mathrm{E}+19$ & $+1-$ & 6.52 \\
\hline $\begin{array}{l}1.000 E-10 \\
1.150 E-07 \\
1.350 E-05 \\
1.000 E-01 \\
1.000 E+00 \\
1.000 E+01 \\
2.000 E+01 \\
4.000 E+01 \\
1.000 E+02 \\
2.000 E+02\end{array}$ & $\begin{array}{l}1.570 \mathrm{E}+10 \\
1.993 \mathrm{E}+11 \\
3.982 \mathrm{E}+12 \\
5.591 \mathrm{E}+12 \\
2.921 \mathrm{E}+12 \\
9.752 \mathrm{E}+10 \\
9.934 \mathrm{E}+10 \\
8.390 \mathrm{E}+10 \\
3.318 \mathrm{E}+10 \\
8.996 \mathrm{E}+10\end{array}$ & $\begin{array}{l}3.055 \mathrm{E}+16 \\
3.880 \mathrm{E}+17 \\
7.751 \mathrm{E}+18 \\
1.088 \mathrm{E}+19 \\
5.686 \mathrm{E}+18 \\
1.898 \mathrm{E}+17 \\
1.934 \mathrm{E}+17 \\
1.633 \mathrm{E}+17 \\
6.459 \mathrm{E}+16 \\
1.751 \mathrm{E}+17\end{array}$ & $\begin{array}{l}+1- \\
+1- \\
+1- \\
+1- \\
+1- \\
+1- \\
+1- \\
+1- \\
+1- \\
+i-\end{array}$ & $\begin{array}{r}28.77 \\
17.70 \\
7.91 \\
5.47 \\
9.00 \\
11.42 \\
11.68 \\
15.88 \\
22.33 \\
21.87\end{array}$ \\
\hline
\end{tabular}

SUMMARY OF INTEGRATED GROUP ENERGY FLUXES AND FLUENCES

\begin{tabular}{|c|c|c|c|c|c|}
\hline $\begin{array}{l}\text { ZNERGY } \\
\text { MeV }\end{array}$ & $\begin{array}{c}\text { FLUX } \\
\mathrm{n} / \mathrm{cm} 2-\mathrm{s}-\mathrm{mA}\end{array}$ & $\begin{array}{c}\text { FLUX } \\
\mathrm{n} / \mathrm{cm} 2-\mathrm{s}\end{array}$ & $\begin{array}{l}\text { FLUENCE } \\
\mathrm{n} / \mathrm{cm} 2\end{array}$ & $\begin{array}{r}\text { STANDARD } \\
\text { DEVIATION }\end{array}$ & के \\
\hline $\begin{array}{c}<1.0 \\
0.1-1.0 \\
\pm .0-10.0 \\
20.0-20.0 \\
20.0-40.0 \\
40.0-100.0 \\
100.0-200.0 \\
>200.0 \\
\text { IOTAL }\end{array}$ & $\begin{array}{l}4.197 E+12 \\
5.591 E+12 \\
2.921 E+12 \\
9.752 E+10 \\
9.934 E+10 \\
8.390 E+10 \\
3.318 E+10 \\
8.996 E+10 \\
1.312 E+13\end{array}$ & $\begin{array}{l}1.289 \mathrm{E}+12 \\
1.718 \mathrm{E}+12 \\
8.974 \mathrm{E}+11 \\
2.996 \mathrm{E}+10 \\
3.052 \mathrm{E}+10 \\
2.578 \mathrm{E}+10 \\
1.019 \mathrm{E}+10 \\
2.764 \mathrm{E}+10 \\
4.031 \mathrm{E}+12\end{array}$ & $\begin{array}{l}\text { 8. } 170 E+18 \\
1.088 E+19 \\
5.686 E+18 \\
1.898 E+17 \\
1.934 E+17 \\
1.633 E+17 \\
6.459 E+16 \\
1.751 E+17 \\
2.554 E+19\end{array}$ & $\begin{array}{l}-1-7.91 \\
-1-5.47 \\
-1-9.00 \\
-1-11.42 \\
-1-11.68 \\
-1-15.88 \\
-1-22.33 \\
-1-21.87 \\
-1-6.52\end{array}$ & 22. \\
\hline
\end{tabular}

THE PERCENTAGE OF NEUTRONS ABOVE $20 \mathrm{MEV}$ IS - 2.33

RELATIVE COVARIANCES (10X10)

$\begin{array}{rrrrrrrrrr}1000 & 889 & 42 & -179 & -204 & -34 & 95 & 51 & 2 & 6 \\ 1000 & 373 & 40 & -38 & -26 & 79 & 65 & -4 & 13 & \\ 1000 & 886 & 794 & 233 & -125 & -71 & 70 & 55 & & \\ 1000 & 945 & 393 & -163 & -151 & 77 & 79 & & \\ 1000 & 567 & -146 & -214 & 82 & 104 & & & \\ 1000 & 489 & -110 & -115 & 109 & & & & \\ 1000 & 593 & -226 & -223 & & & & & \\ 1000 & 290 & -563 & & & & & & \\ 1000 & -127 & & & & & & & \\ 1000 & & & \end{array}$

